\title{
Exploring the mechanism of SHP2 and EGFR/HER2 cooperation in breast cancer cell signaling
}

\author{
Zachary Raymond Hartman \\ West Virginia University
}

Follow this and additional works at: https://researchrepository.wvu.edu/etd

\section{Recommended Citation}

Hartman, Zachary Raymond, "Exploring the mechanism of SHP2 and EGFR/HER2 cooperation in breast cancer cell signaling" (2013). Graduate Theses, Dissertations, and Problem Reports. 396.

https://researchrepository.wvu.edu/etd/396

This Dissertation is protected by copyright and/or related rights. It has been brought to you by the The Research Repository @ WVU with permission from the rights-holder(s). You are free to use this Dissertation in any way that is permitted by the copyright and related rights legislation that applies to your use. For other uses you must obtain permission from the rights-holder(s) directly, unless additional rights are indicated by a Creative Commons license in the record and/ or on the work itself. This Dissertation has been accepted for inclusion in WVU Graduate Theses, Dissertations, and Problem Reports collection by an authorized administrator of The Research Repository @ WVU.

For more information, please contact researchrepository@mail.wvu.edu. 


\title{
Exploring the mechanism of SHP2 and EGFR/HER2 cooperation in breast cancer cell signaling
}

\author{
Zachary Raymond Hartman
}

\author{
Dissertation submitted to the \\ School of Medicine \\ at West Virginia University
}

in partial fulfillment of the requirements for the degree of Doctor of Philosophy in Biochemistry and Molecular Biology

\author{
Yehenew Agazie, Ph.D., Chair \\ Peter Gannett, Ph.D. \\ Michael Schaller, Ph.D. \\ John M. Ruppert, Ph.D. \\ Xiaodong Shi, Ph.D.
}

Dissertation defense date: November 21, 2013

Keywords: Breast cancer, SHP2, cell signaling 


\section{Abstract}

\section{Exploring the mechanism of SHP2 and EGFR/HER2 cooperation in breast cancer cell signaling}

\section{Zachary Raymond Hartman}

The Src homology 2-containing protein tyrosine phosphatase 2 (SHP2) has been established as a critical mediator of cancer-related cell signaling downstream of receptor tyrosine kinases like EGFR and HER2. As such, targeting of SHP2 is being recognized as a potentially viable therapeutic option in HER2-positive and triple-negative breast cancers. In this report, I employed molecular and cellular biology techniques to further elucidate the role of SHP2 downstream of receptor tyrosine kinases in breast cancer cell lines. In this way, I was able to contribute knowledge to how SHP2 controls triple-negative cell motility. In addition, the mechanism of SHP2 control of HER2 signaling was investigated in order to better understand how SHP2 positively mediates signaling. The EGFR/HER2 heterodimerization axis was also explored, uncovering a mechanism by which HER2 is able to protect EGFR from degradation through suppression of phosphorylation at specific sites. Finally, molecular modeling was used to determine how SHP2 is able to selectively interact with endogenous substrates. These studies contribute both to the rationale for targeting SHP2 and the mechanism by which SHP2 promotes breast cancer signaling in cooperation with EGFR and HER2. The knowledge added by these studies will hopefully contribute in the future toward the development of selective small molecule inhibitors of SHP2. 


\section{Acknowledgements}

It seems to me that the list of people who have contributed in some way to this dissertation could fill its own thesis. Unfortunately, brevity is king in science, and so I must try and distill the acknowledgements appropriately.

First and foremost, my mentor, Yehenew Agazie, has provided the foundation for my training and future career path. It was he, more than anyone else, who supplied the formative training in writing and critical thought that I need moving forward. I will forever be indebted to him for taking me into his lab and letting me work on the very interesting projects described herein. Though I am still far from a perfect writer and thinker, Yehenew has taught me to value patience and perseverance above all.

The other members of my committee have all contributed greatly to my development. Without Dr. Gannett, I would not have ever had the opportunity to learn molecular modeling and pursue this educational path. Without my conversations with Dr. Schaller, I might still be stuck on a few key experiments. Dr. Ruppert taught me much about cancer and provided me with some encouraging and colorful advice best left out of a formal manuscript. Dr. Shi gave me a profound appreciation for stretching into learning difficult concepts outside one's field of interest. Each member gave significant time and energy for my benefit, and I cannot overstate the value of my committee.

My peers in the Biochemistry and Cancer Cell Biology Departments have also given me so much that neither they nor I can properly recognize. Conversations, therapeutic complaint sessions, and camaraderie all kept life in graduate school to a bare minimum of insanity. I reserve a special place in my heart for my former lab mates, Yan Wang, Fatimah Matalkah, and Elisha Martin. In different ways, they were all phenomenal, and I was able to learn a lot about them just by watching and talking.

My wife, Chelsey, has had a lot to put up with during this entire process. She, more than anyone else, has had to share in my scientific failures. However, few things can get your spirit back to par faster than knowing you have someone watching your back. That's what I have in my wife, and I don't know how I would have fared or who I would be today without her.

My family members, especially my parents, have been completely immovable boulders of support throughout my entire life. Their sacrifice put me here. If I can find a way to work half as hard as they, then my success will be all but guaranteed. 


\section{Tables of Contents}

1.1) BREAST CANCER PUBLIC HEALTH SIGNIFICANCE 9

$\begin{array}{lr}1.2) \text { BREAST CANCER PATHOGENESIS } & 9\end{array}$

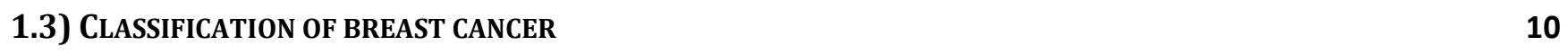

$\begin{array}{lr}\text { 1.3.1) HER2-POSITIVE BREAST CANCER } & 11\end{array}$

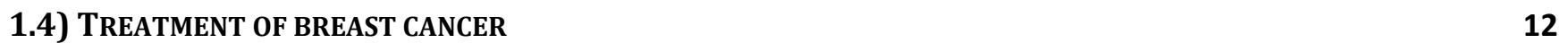

$\begin{array}{ll}\text { 1.4.1) SURGERY AND RADIOTHERAPY } & 13\end{array}$

$\begin{array}{ll}\text { 1.4.2) SYSTEMIC, CYTOTOXIC CHEMOTHERAPY } & 13\end{array}$

$\begin{array}{ll}\text { 1.4.3) SYSTEMIC, TARGETED THERAPIES } & 13\end{array}$

2) THE TYROSINE PHOSPHATASE SHP2 $\quad 16$

2.1) PROTEIN TYROSINE PHOSPHATASES: INTRODUCTION AND CATALYTIC MECHANISM 16

2.2) SHP2 DISCOVERY AND INITIAL CHARACTERIZATION $\quad 17$

2.3) STRUCTURAL REGULATION OF SHP2 18

2.4) SHP2 IN NORMAL CELL SIGNALING $r$

$\begin{array}{ll}\text { 2.4.1) SHP2 IN MITOGENIC SIGNALING } & 19\end{array}$

$\begin{array}{ll}\text { 2.4.2) SHP2 IN CELL SURVIVAL } & 21\end{array}$

$\begin{array}{ll}\text { 2.4.3) SHP2 IN CELL FATE } & 23\end{array}$

$\begin{array}{ll}2.4 .4) \text { SHP2 IN CELL MOTILITY } & 23\end{array}$

2.5) SHP2 IN PATHOLOGY $r$

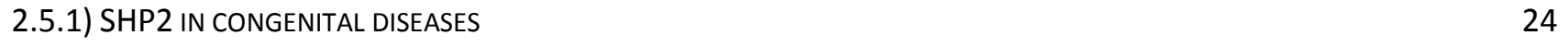

2.5.2) SHP2 IN HEMATOPOIETIC MALIGNANCY $\quad 25$

$\begin{array}{ll}2.5 .3) \text { SHP2 IN SOLID TUMORS } & 26\end{array}$

3) HER2 STABILIZES EGFR AND ITSELF BY ALTERING AUTOPHOSPHORYLATION PATTERNS IN A MANNER THAT OVERCOMES REGULATORY MECHANISMS AND PROMOTES PROLIFERATIVE AND TRANSFORMATION SIGNALING29

3.1) ABSTRACT 29

3.2) INTRODUCTION 30

3.3) MATERIALS AND METHODS 
3.3.1) CELLS, CELL CULTURE AND REAGENTS

3.3.2) SUBCLONING, RETROVIRAL TRANSDUCTION AND PRODUCTION OF STABLE CELL LINES 32

3.3.3) STABLE ANTI-EGFR SHRNA EXPRESSION

3.3.4) PREPARATION OF CELL LYSATES, GEL ELECTROPHORESIS AND IMMUNOSTAINING ANALYSIS

3.3.5) IMMUNOPRECIPITATION STUDIES

$\begin{array}{ll}\text { 3.3.6) ANCHORAGE-INDEPENDENT GROWTH ASSAY } & 35\end{array}$

3.3.7) TRITC-LABELED EGF STUDIES

$\begin{array}{ll}\text { 3.3.8) IN VIVO TUMORIGENESIS STUDIES } & 36\end{array}$

3.4) RESULTS

3.4.1) HER2 OVEREXPRESSION DOES NOT LEAD TO RECEPTOR HYPERPHOSPHORYLATION 36

3.4.2) HER2 ALTERS AUTOPHOSPHORYLATION PATTERNS IN A MANNER THAT CONFERS DIFFERENTIAL INTERACTION 38

3.4.3) C-CBL IS UNABLE TO INTERACT WITH THE EGFR:HER2 HETERODIMER, LEADING TO REDUCED UBIQUITINYLATION 40

3.4.4) HER2-INDUCED INHIBITION OF GRB2-BINDING SITE AUTOPHOSPHORYLATION DOES NOT PERTURB SIGNALING 41

3.4.5) Y1068F-EGFR INDUCES SUSTAINED SIGNALING COMPARED TO THE WILD-TYPE COUNTERPART 42

3.4.6) EGF-INDUCED Y1068F-EGFR TRAFFICKING RESEMBLES THAT OF WT-EGFR IN PRESENCE OF HER2 43

3.4.7) Y1068F-EGFR IS MORE TRANSFORMING WHEN COMPARED TO THE WILD-TYPE COUNTERPART

3.4.8) HER2 EXPLOITS THE EGFR TO ENHANCE ITS TUMORIGENIC POTENTIAL

$\begin{array}{ll}3.5) \text { DISCUSSION } & 46\end{array}$

3.6) ACKNOWLEDGEMENT $r$

$\begin{array}{lr}\text { 3.7) FIGURE LEGENDS } & 52\end{array}$

4) THE TYROSINE PHOSPHATASE SHP2 REGULATES FOCAL ADHESION KINASE TO PROMOTE EGF-INDUCED LAMELLIPODIA PERSISTENCE AND CELL MIGRATION $\quad 66$

$\begin{array}{lr}\text { 4.1) ABSTRACT } & 67\end{array}$

$\begin{array}{lr}\text { 4.2) INTRODUCTION } & 68\end{array}$

$\begin{array}{lr}\text { 4.3) Materials AND METHODS } & \mathbf{7 0}\end{array}$

$\begin{array}{ll}\text { 4.3.1) CELLS AND REAGENTS } & 70\end{array}$

4.3.2) SILENCING SHP2 EXPRESSION WITH SHRNA $\quad 70$

4.3.3) SITE-DIRECTED MUTAGENESIS, SUBCLONING AND EXPRESSION
71

4.3.4) PREPARATION OF CELL LYSATES, IMMUNOPRECIPITATION AND IMMUNOSTAINING

4.3.5) MONOLAYER WOUND-HEALING ASSAY

$\begin{array}{ll}\text { 4.3.6) IMMUNOFLUORESCENCE } & 73\end{array}$

$\begin{array}{ll}\text { 4.3.7) ASSAY FOR GOLGI ORIENTATION } & 73\end{array}$

$\begin{array}{ll}\text { 4.3.8) LIVE-CELL IMAGING AND KYMOGRAPHIC ANALYSIS } & 73\end{array}$

4.3.9) SUBSTRATE-TRAPPING, AFFINITY PRECIPITATION AND FAR WESTERN ANALYSIS

$\begin{array}{ll}\text { 4.3.10) IN VITRO PHOSPHATASE ASSAY } & 74\end{array}$

$\begin{array}{ll}\text { 4.3.11) STATISTICAL ANALYSES } & 75\end{array}$

$\begin{array}{ll}\text { 4.4) RESULTS } & 75\end{array}$

4.4.1) SHP2 IS REQUIRED FOR BASAL AS WELL AS EGF-STIMULATED BTBC CELL MOTILITY

4.4.2) SHP2 PROMOTES PROPER CELL DIRECTIONALITY DURING WOUND HEALING

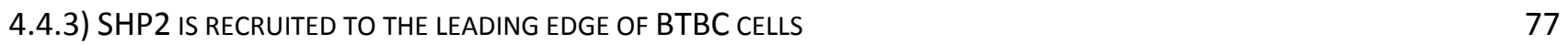


4.4.4) SHP2 DEPLETION ALTERS EGF-STIMULATED MEMBRANE PROTRUSIONS IN BTBC CELLS 78

$\begin{array}{ll}\text { 4.4.5) PY397 IN FAK IS A TARGET FOR SHP2 PTPASE ACTIVITY } & 79\end{array}$

4.4.6) SHP2 PROMOTES FAK INACTIVATION TO PROMOTE EGF-STIMULATED WOUND HEALING 81

4.5) DISCUSSION $\quad 84$

4.6) ACKNOWLEDGEMENTS $\quad 87$

$\begin{array}{lr}\text { 4.7) FIGURE LEGENDS } & 87\end{array}$

5) SUBSTRATE DISCRIMINATION BY THE SRC HOMOLOGY 2-CONTAINING PROTEIN TYROSINE PHOSPHATASE SHP2 \begin{tabular}{lr} 
PREDICTED BY MOLECULAR DOCKING & 97 \\
\hline
\end{tabular}

$\begin{array}{lr}\text { 5.1) ABSTRACT } & 97\end{array}$

$\begin{array}{lr}\text { 5.2) INTRODUCTION } & 97\end{array}$

5.3) MATERIALS AND METHODS $\quad 99$

$\begin{array}{ll}\text { 5.3.1) Cells, CELL CULTURE, AND REAGENTS } & 99\end{array}$

5.3.2) PURIFICATION OF RECOMBINANT GST FUSION PROTEINS 99

$\begin{array}{ll}\text { 5.3.3) IN VITRO PHOSPHATASE ACTIVITY ASSAY } & 100\end{array}$

5.3.4) SITE-DIRECTED MUTAGENESIS, SUBCLONING, AND EXPRESSION OF HER2 100

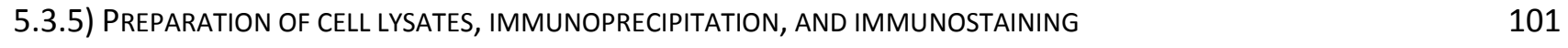

$\begin{array}{ll}\text { 5.3.6) AFFINITY PRECIPITATION } & 102\end{array}$

$\begin{array}{ll}\text { 5.3.7) ANCHORAGE-INDEPENDENT GROWTH ASSAY } & 102\end{array}$

$\begin{array}{ll}\text { 5.3.8) MOLECULAR DOCKING } & 103\end{array}$

$\begin{array}{lr}\text { 5.4) RESULTS } & 103\end{array}$

$\begin{array}{ll}\text { 5.4.1) A SUBSTRATE-DERIVED PEPTIDE IS A SELECTIVE SHP2 INHIBITOR } & 103\end{array}$

5.4.2) MUTATION OF HER2 ABOLISHES INTERACTION WITH SHP2 105

5.4.3) DISRUPTION OF HER2-SHP2 INTERACTION REDUCES TRANSFORMATION BY HER2 106

5.4.4) MOLECULAR MODELING PREDICTS BINDING DETERMINANTS OF SHP2 AND SUBSTRATE 107

$\begin{array}{lr}\text { 5.5) DISCUSSION } & 109\end{array}$

5.6) FIGURE LEGENDS $r 11$

6) DISCUSSION 121

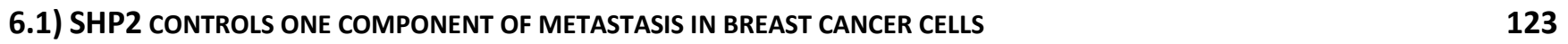

$\begin{array}{lr}\text { 6.2) DESIGN OF SELECTIVE SHP2 INHIBITORS IS POSSIBLE } & 125\end{array}$

$\begin{array}{lr}\text { 6.3) PROPOSED EXPERIMENTS } & 130\end{array}$

$\begin{array}{lr}6.4) \text { CONCLUSIONS } & 132\end{array}$

\begin{tabular}{lr} 
7) REFERENCES & 132 \\
\hline
\end{tabular}

8) CURRICULUM VITAE 141 


\section{Glossary}

Adenosine triphosphate

ATP

Basal-like and triple negative breast cancer

BTBC

Bovine serum albumin

BSA

Complementary DNA

cDNA

Corkscrew phosphatase

Csw

Double-mutant SHP2

Epidermal growth factor

DM-SHP2

Epidermal growth factor receptor 1

EGF

Extracellular signal-related kinase 1/2

EGFR

Focal adhesion kinase

ERK1/2

Fibroblast growth factor

FAK

Growth factor receptor-bound 2

FGF

Glutathione S-transferase

Grb2

Guanosine triphosphate

GST

Guanosine diphosphate

GTP

GTP phosphatase

Human epidermal growth factor receptor 2

GDP

Kilodalton

GTPase

HER2

Mitogen-activated protein kinase

Murine embryonic fibroblast

$\mathrm{kDa}$

MAPK

Phosphate-buffered saline

MEF

Platelet-derived growth factor

PBS

Phosphoinositide-3 kinase

PDGF

Phosphotyrosine binding

PI3K

Protein tyrosine phosphatase

PTB

PTP

Phosphotyrosine

pY

Ras GTPase-activating protein

RasGAP

Receptor tyrosine kinase

RTK

Src homology 2

$\mathrm{SH} 2$

$\mathrm{SH} 2$ domain-containing protein tyrosine phosphatase 1

SHP1

SH2 domain-containing protein tyrosine phosphatase 2

SHP2

Short hairpin RNA

shRNA

Son of Sevenless

SOS

Tris-buffered saline with Tween-20

TBST

Wild-type

WT 


\section{List of figures}

Literature review

1. PTP catalytic mechanism

2. Mechanism of SHP2 structural regulation

3. Pathways impacted by SHP2 signaling

$\underline{\text { Study I }}$

1. Impact of SHP2 inhibition on wound healing and golgi body orientation

2. SHP2 localizes to the leading edge of BTBC cells

3. Impact of SHP2 silencing on EGF-induced wound healing and lamellipodial persistence

4. SHP2 regulates FAK activity in BTBC cells

5. Analysis of impact of SHP2 silencing on the pY397-FAK levels

6. Effect of FAK inhibition of EGF-induced wound healing, lamellipodial persistence, and Golgi orientation

Study II

1. Derivation of a substrate-derived peptide that selectively inhibits SHP2

2. Mutation of HER2 abolishes interaction with SHP2

3. Mutation of HER2 results in attenuated response to EGF stimulation and inhibits transformation

4. Predicted binding more of substrate-derived peptide to the SHP2 active site

5. Predicted binding mode of substrate-derived peptide to the SHP1 active site Study III

1. Analysis of EGF-induced receptor autophosphorylation

2. Analysis of specific autophosphorylation and interaction

3. Analysis of EGFR and HER2 coprecipitation with c-Cbl in the MCF-10A

4. Impact of HER2 expression on signaling

5. Mutation of the Grb2 binding site in EGFR and HER2 does not perturb signaling 
6. HER2 exploits the EGFR to enhance its tumorigenic potential

\section{Introduction}

\section{1) Breast cancer public health significance}

Breast cancer is one of the most significant public health concerns for women in the world. In US women, it is the most common form of malignant neoplasm, and, after lung cancer, it is responsible for the most cancer-related mortality. The American Cancer Society estimates that the number of new breast cancer diagnoses will reach 232,340 in the year 2013, and a total of 39,620 deaths will result from breast cancer (1). Prevalence generally increases with age; $78 \%$ of all new diagnoses in 2013 occurred in women above the age of 50 (1). Other significant risk factors for developing breast cancer include high breast density, family history of breast cancer, alcohol consumption, early menarche, and obesity, among others (1).

Breast cancer treatment incurs nearly $\$ 4.2$ billion per year in the US in direct costs (2). The indirect costs-time lost from work due to disability or mortality and social impact, etc. are more difficult to estimate, but one study has placed this burden in terms of "short-term" (i.e., 12 month) disability and sick leave costs at approximately $\$ 3690$ per patient with primary breast cancer and $\$ 6166$ per patient with metastatic disease (3). From these data, it is clear that breast cancer represents a profound societal burden.

\section{2) Breast cancer pathogenesis}

Breast cancer, like other forms of cancer, is thought to result from genetic changes in normal cells that enhance proliferation and confer escape from physiologic programmed cell death (4). The majority ( $\sim 95 \%$ ) of breast cancer cases are sporadic in nature, resulting from genetic aberrations that accumulate over time due to environmental factors such as exposure to 
carcinogens. The remaining $5 \%$ of breast cancers are hereditary in nature, generally resulting from inactivating mutations in the $B R C A-1$ and $B R C A-2$ genes that encode E3 ubiquitin ligases used in DNA repair (4). Dysfunction of the normal DNA damage response can promote genomic instability, increasing the likelihood of mutations that activate proto-oncogenes or that inactivate tumor suppressors (4). Accumulation of these mutations can confer the hallmarks of cancer: sustained proliferation, evasion of cell cycle control, promotion of metastasis, replicative immortality, promotion of angiogenesis, and resistance to apoptosis (5).

\section{3) Classification of breast cancer}

The discovery that ovarectomy, removal of the ovaries to terminate estrogen production, was an effective treatment for some, but not all cases of breast cancer indicated that the disease was heterogeneous (6). Today, clinicians rely on several techniques to determine the phenotype of breast cancer. Immunohistochemistry is used to assess the expression of the hormone receptors (estrogen receptor and progesterone receptor, ER and PR) and HER2 (human epidermal growth factor receptor 2, a proto-oncogenic receptor tyrosine kinase). In cases where HER2 expression is equivocal, fluorescence in situ hybridization is used to directly determine the copy number of the gene. These data allow clinicians to stratify cases of breast cancer into three major types: hormone receptor-positive, HER2-positive, and triple-negative (7).

Recent advances in breast cancer molecular biology has led to the classification of breast cancer into five distinct subtypes based on gene expression profiling rather than immunohistochemistry and copy number. These include luminal A and luminal B (with a gene expression signature matching the hormone receptor-positive luminal cells of the breast), 
HER2-positive, basal-like (with a gene expression signature matching features of the myoepithelial cells of the basal lamina, usually lacking expression of either hormone receptors or HER2), and normal-like (with a gene expression signature clustered tightly with the normal basal epithelium) (8).

The hormone receptor-positive disease is the most common form of breast cancer, representing approximately $60 \%$ of all cases of breast cancer. HER2-positive disease accounts for $15-20 \%$ of all cases (9), while the triple-negative disease accounts for another $15-20 \%$ of all cases. Up to $85 \%$ of triple-negative cancers express basal myoepithelial markers like cytokeratins 5 and $6(10)$.

\subsection{1) HER2-positive breast cancer}

HER2 is the most commonly-dysregulated proto-oncogenic tyrosine kinase that drives breast cancer. When it was first characterized, the hormone-dependent forms of breast cancer had been effectively treated for nearly two decades (11). HER2 was discovered following transformation of normal mouse fibroblasts using DNA harvested from a rat neuroblastoma (12). This oncogenic DNA was found to encode a $185 \mathrm{kDa}$ protein (13), and the responsible gene was isolated and termed $\mathrm{Neu}(14)$. The human homologue of $\mathrm{Neu}$ was found a year later, and its extensive sequence homology to the epidermal growth factor receptor (EGFR) led scientists to name the protein "human epidermal growth factor receptor 2," HER2 (15). Throughout the late 1980s, HER2 was characterized as an important driving force in breast cancer, mainly through its gene amplification on Chromosome 17 (16).

The oncogenicity of HER2 results from dysregulation of its ligand binding domain. Normally, EGFR family members require the binding of a ligand to promote dimerization and 
signal transduction. However, the ligand binding domain of HER2 is constitutively blocked by interactions with other nearby domains. This results in an altered conformation of the dimerization arm that allows it to associate with other activated EGFR family members (17). Overexpression of HER2 promotes the ligand-independent formation of homo- or heterodimers. In this way, HER2 can act as a strong potentiator of mitogenic and survival signaling, leading to cancer growth and poor prognosis if left untreated (18).

While it can portend poor outcomes for patients, HER2 overexpression presents two unique opportunities for therapy. First, since cancer cells rely on upregulated HER2 for growth and survival, inhibition of its activity may selectively harm tumors while largely sparing the normal cells in the body. Second, since HER2 possesses a unique extracellular domain, a targeted agent may not need to enter the cell to inhibit its function. This was demonstrated for the first time in the 1986 when a monoclonal antibody developed against the extracellular region of HER2 was found to inhibit growth of HER2-transformed cells (19). This observation led to the development of the humanized antibody trastuzumab, which became the first therapy designed to target a single molecule in breast cancer (20). The efficacy of trastuzumab will be discussed further below.

\section{4) Treatment of breast cancer}

When breast cancer is diagnosed, therapy will be influenced by factors that include the stage of the disease (i.e., metastatic versus early-stage disease), tumor grade, and subtype. The strategies at clinicians' disposal include gross techniques such as surgery and radiotherapy as well as systemic chemotherapies. 


\subsection{1) Surgery and radiotherapy}

Thanks to improvements in early breast cancer detection, most breast tumors are discovered in the pre-metastatic setting (4). Therefore, many patients benefit from resection in the form of lumpectomy (removal of tumor sparing the rest of the breast as much as possible) or mastectomy (removal of a significant portion or the entirety of the breast). Radiation is often prescribed to help eliminate cells that may have escaped surgical removal.

\subsection{2) Systemic, cytotoxic chemotherapy}

As an additional regimen to suppress disease relapse, cytotoxic chemotherapy is often prescribed after surgery (the so-called adjuvant setting). These agents target rapidly-dividing cells to promote programmed cell death. There are many types of chemotherapeutics available to clinicians, including anthracyclines, which intercalate into DNA and RNA to prevent replication, alkylating agents, which damage the DNA via alkylation, antimetabolites, which interfere with the synthesis of DNA and RNA building blocks, and microtubule inhibitors, which disrupt the functions of microtubules to prevent mitosis. Each of these drug families can reduce tumor size and help to prolong survival. Agents are often combined since their mechanisms of action and toxicities do not overlap. Nevertheless, cytotoxic chemotherapy is highly toxic, producing side effects ranging from the relatively-benign such as hair loss to the severe and life-threatening such as cardiotoxicity and sepsis (21).

\subsection{3) Systemic, targeted therapies}

Researchers have long sought a "magic bullet" for cancer to take advantage of the genetic aberrations that drive tumor growth and selectively induce apoptosis in cancer cells. To this end, many agents have been developed to target specific subtypes of breast cancer. Hormone 
receptor-positive disease can be treated with selective estrogen receptor modifying drugs such as tamoxifen, a competitive inhibitor targeting the receptor, and anastrazole, an aromatase inhibitor that disrupts the production of estrogen. HER2-positive disease has been targeted by the biologic trastuzumab (Herceptin, Roche). Small molecule inhibitors against the HER2 kinase domain like lapatinib (Tykerb, GlaxoSmithKline) have also been approved for use in HER2-positive disease.

Targeted therapeutics produce significant benefit for many patients. For example, trastuzumab was shown in its pivotal trial to dramatically improve response rate when combined with a combination anthracyline-cyclophosphamide regimen (38\% response rate with no added trastuzumab versus 50\% with) and with paclitaxel (15\% response rate without trastuzumab versus $38 \%$ with) (20). As a result, overall survival was improved in metastatic breast cancer by 4-9 months due to addition of trastuzumab. Since metastatic breast cancer is regarded as incurable, these improved response rates and overall survival benefits have made trastuzumab a part of the standard of care for HER2-positive disease.

While these targeted agents have improved breast cancer therapy, problems persist. First, response to therapy is far from a guarantee. Nearly half of patients with clinicallydiagnosed HER2-positive disease fail to respond to trastuzumab therapy (20). This observation reflects heterogeneity of breast cancers even within subtypes, and the mechanism of this de novo resistance to trastuzumab has yet to be elucidated, though the dichotomy between HER2-expressing and HER2-enriched has been forwarded by large-scale analysis of breast samples (22). Specifically, only half of the profiled breast tumors that were clinically diagnosed as HER2-positive showed enrichment of HER2 mRNA, suggesting they would see 
limited benefit to trastuzumab therapy. This conclusion has yet to be formally tested in a prospective clinical trial, however. Second, acquired resistance is common in targeted therapeutics. Biochemical evidence for this phenomenon (e.g., downregulation of the estrogen receptor with treatment of tamoxifen) can be observed as early as the first stages of drug response (4). With small molecule inhibitors like lapatinib, cancer cells can acquire mutations in the target protein that make it resistant to binding by the inhibitor (e.g., mutation of the ATP binding site). Acquired trastuzumab resistance can result from upregulation of secretases such as ADAM10 that promote ectodomain shedding (23) or from switching to reliance upon other receptor tyrosine kinases to promote growth (24).

Finally, no targeted therapies exist to address triple-negative breast tumors. Nonspecific therapies remain the only option. In addition, since triple-negative disease tends to be less differentiated, it is often higher grade, as well, raising the likelihood for disease progression and metastasis (10). Therefore, researchers are exploring many routes to develop triple-negative cancer-specific therapies. Various agents in clinical trials include inhibitors targeting other receptor tyrosine kinases (e.g., erlotinib and pertuzumab that target EGFR), PI3K, and chaperones like Hsp90. In support of this approach, a significant proportion of triple-negative cancers overexpress EGFR (between 50-70\%, depending on the technique used to assess expression) (10).

The challenges associated with therapy in breast cancer cases that involve receptor tyrosine kinase signaling, HER2-positive and triple-negative disease, point to possibilities in targeted therapy downstream of receptors. The remainder of this report focuses on the Src homology 2-containing protein tyrosine phosphatase (SHP2), since it has been shown to 
promote signaling and transformation downstream of oncogenic receptor tyrosine kinases like HER2 (25). As such, it is being recognized as a master regulator of pro-tumorigenic signaling that is an attractive target for therapy in different forms of cancer, including breast cancer (26). Breast cancer, in particular, has been shown to overexpress either SHP2 protein or effectors that contribute to its activation $(27,28)$.

\section{2) The tyrosine phosphatase SHP2}

\section{1) Protein tyrosine phosphatases: introduction and catalytic mechanism}

Phosphorylation of serine, threonine, and tyrosine residues governs many cellular functions. Many oncogenes characterized so far promote tyrosine phosphorylation (e.g., Src kinase and HER2), making this phenomenon especially important in oncology.

Cellular levels of tyrosine phosphorylation are governed by the interplay between kinases and phosphatases, which add or remove phosphate groups, respectively. Tyrosine kinases are pivotal in the transduction of proliferative signals and are common drivers of cancer development and progression (29). To this end, therapeutic targeting of tyrosine kinases is an intense focus of the pharmaceutical industry, occupying $50-70 \%$ of all cancer drug development efforts (30).

Tyrosine phosphatases counter the action of tyrosine kinases. These enzymes include two major families: receptor-type (RTPTP) and cytosolic protein tyrosine phosphatases (PTP). PTPs comprise more than 100 different members that have a highly-conserved active site motif defined by the sequence HCXXGXXRS (Fig. 1) (31,32). When a phosphotyrosine residue 
or similar substrate enters the active site, the conserved arginine helps to attract and position the negatively-charged phosphate moiety (Fig. 1). Histidine coordinates with the catalytic cysteine thiol, lowering its $\mathrm{pK}_{\mathrm{a}}$ and helping to form a thiolate. Nucleophilic attack of the phosphate facilitated by an aspartic acid from the WPD loop, acting as a proton donor, results in the formation of a thiol phosphate intermediate and a free tyrosine residue that diffuses from the active site. The deprotonated aspartate then functions as a general base to catalyze hydrolysis of the intermediate, releasing inorganic phosphate and resetting the enzyme (32).
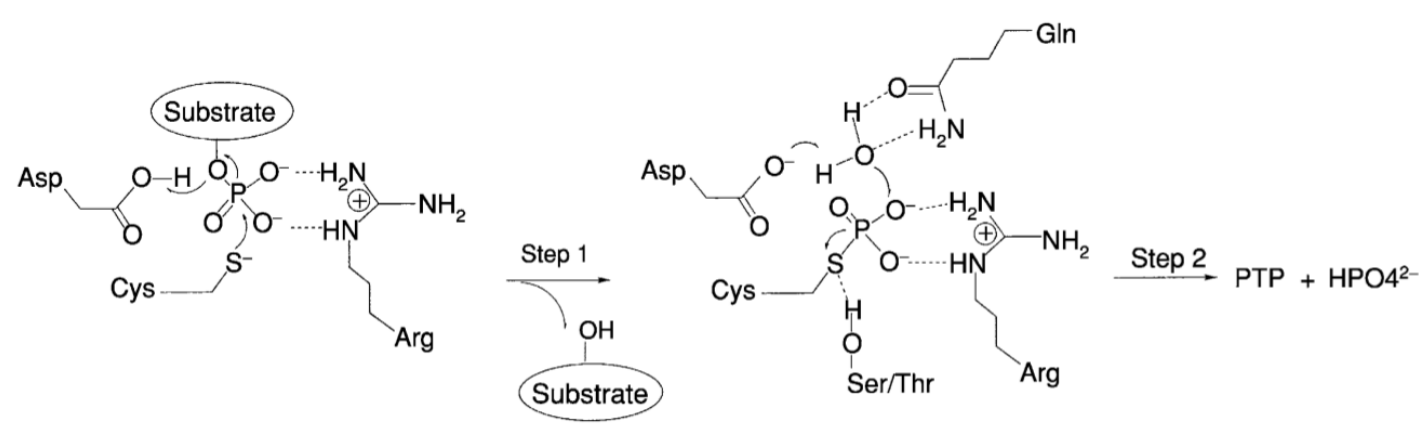

Figure 1. PTP catalytic mechanism. Nucleophilic attack by the catalytic cysteine is facilitated by the aspartate of the WPD loop and the arginine of the catalytic motif followed by hydrolysis catalyzed by the aspartate to yield inorganic phosphate and a free enzyme (33).

\section{2) SHP2 discovery and initial characterization}

Throughout the 1980s and into the early 1990s, mediators of EGFR and other receptor tyrosine kinases were discovered and characterized in Drosophila. One such gene, Corkscrew (Csw), was identified as a mutation in the receptor tyrosine kinase Torso pathway. Csw was shown to promote the activation of this signaling parthway (31). In 1992 and 1993, the mammalian ortholog was discovered by five different groups, each giving the protein product a 
different name (Syp, SH-PTP2, SH-PTP3, PTP1D, and PTP2C) (34-38). Researchers noted the sequence similarity between this new tyrosine phosphatase and the Csw gene product, suggesting that a mammalian phosphatase could play a positive role in signaling (35). The naming convention of the protein was eventually unified as the SH2-containing protein tyrosine phosphatase 2 (SHP2); this standardization was formalized in a letter to Cell in 1994. (Dr. Benjamin Neel, personal communication)

\section{3) Structural regulation of SHP2}

Aside from its active site, SHP2 has several important structural features. It contains two SH2 domains arranged tandemly that help to regulate SHP2 activity (29). The N-terminal SH2 domain (N-SH2) sits atop the active site, preventing access by phosphotyrosine substrates (39). When the N-SH2 domain engages a phosphotyrosine, conformational changes within the SH2 domain weaken the association with the active site, promoting an open conformation that is exposed to substrates (Fig. 2). The N-SH2 domain acts as both an activity gatekeeper and a trafficking regulator, promoting SHP2 localization to areas of high phosphotyrosine content. This dichotomy between the "closed" and "open" configurations of SHP2 is thought to be the most important means of regulating activity. The C-terminal SH2 domain does not function as a direct inhibitor of the active site in the same way. It is thought to further promote SHP2 trafficking; however, its full function is not known. 


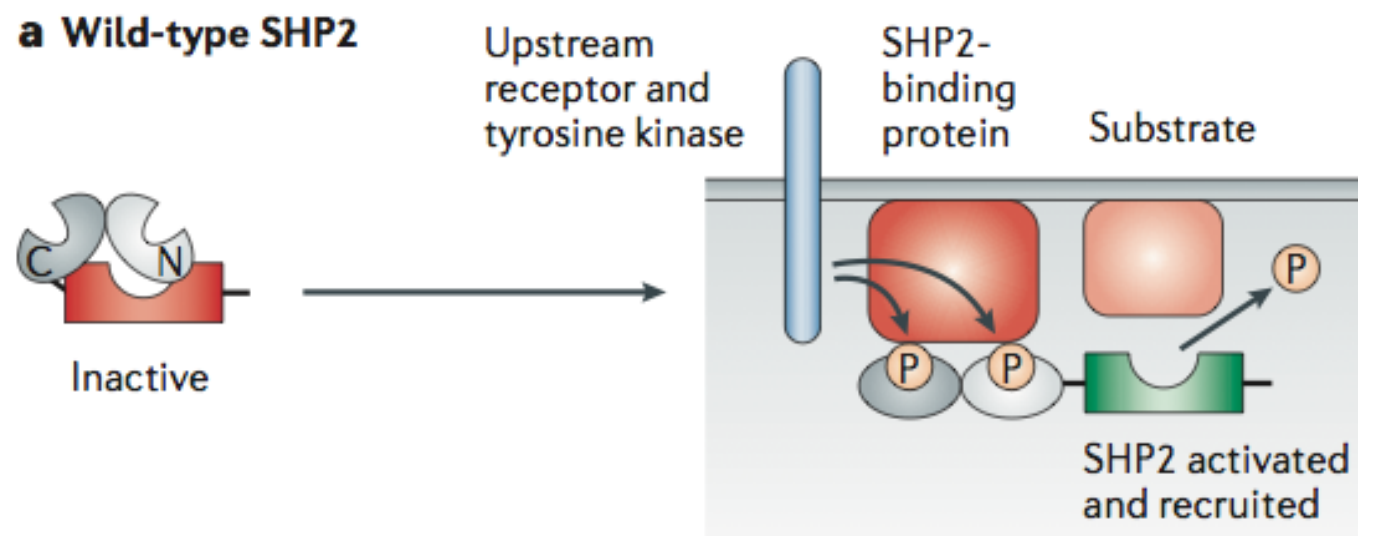

Figure 2. Mechanism of SHP2 structural regulation. SHP2 is kept in an inactive, "closed" conformation under normal conditions. Activation of receptors in the cell results in upregulation of membrane tyrosine phosphorylation that promotes SHP2 translocation. Engagement of the SH2 domains weakens the association of the N-terminal SH2 domain with the catalytic domain, rendering SHP2 active near its substrates (40).

SHP2 also contains a C-terminal tail with two phosphorylatable tyrosine residues. When phosphorylated, they are able to act as docking sites for SH2-containing proteins such as growth factor receptor-bound 2 (Grb2) (41). When these tyrosines are mutated to phenylalanine, fibroblast growth factor and platelet-derived growth factor-induced signaling are inhibited (41). A proline-rich region is also located in the C-terminus, but its function has yet to be elucidated (26).

\section{4) SHP2 in normal cell signaling}

\subsection{1) SHP2 in mitogenic signaling}

SHP2 and its orthologues (i.e., Csw in D. melanogaster) are essential for the development of numerous animal models, including C. elegans (42), D. melanogaster (43), X. laevis (44), and M. musculus (45). The primary role of SHP2 in these systems appears to be control of Rasmediated mitogenic signaling downstream of growth factor receptors. Among the first of these 
growth factor receptor pathways shown to be SHP2 dependent was that of the platelet-derived growth factor receptor (PDGFR) (46). SHP2 control of this signaling pathway is multifactorial (Fig. 3). First, SHP2 directly controls phosphorylation levels of the receptor following stimulation of cells with PDGF (46). In addition, SHP2 can become phosphorylated in response to PDGF (41). Phosphorylation of both tyrosine residues of SHP2's C-terminal tail is required for maximal induction of mitogen-activated protein kinase (MAPK) signaling in response to PDGF. Mutation of these residues, however, did not abolish activation of the pathway promoted by SHP2, suggesting other mechanisms of regulation. Analysis of the Torso pathway, the near-equivalent of PDGFR in D. melanogaster, provides a clue into the other roles of SHP2 in PDGF signaling. Csw dephosphorylates the phosphotyrosine responsible for recruiting the Drosophila homologue to the Ras GTPase activating protein (RasGAP), regulating signaling in a manner similar to EGFR in mammalian cells, discussed below (47).

Other extensively-studied growth factor cascades mediated by SHP2 include the fibroblast growth factor (FGF) and epidermal growth factor (EGF) pathways. Signaling is initiated by ligand binding to the receptor, promoting receptor dimerization. This induces conformational changes in the juxtamembrane domain, positioning the kinase domain of one molecule near the C-terminal tail of its partner and inducing phosphorylation of multiple tyrosine residues. Phosphorylation allows for the recruitment of SH2 domain-containing molecules that act as either enhancers or inhibitors of signaling. For example, Grb2 contains an SH2 domain that binds to phosphotyrosines of EGFR, and it acts as a scaffold that recruits the Son of Sevenless (SOS), allowing the latter's pleckstrin homology domain to interact with nearby phospholipids, relieving SOS autoinhibition. This promotes the guanine exchange factor activity of SOS, converting inactive Ras-GDP into the active Ras-GTP and resulting in 
subsequent activation of the MAPK pathway (48). At the same time, negative regulatory proteins are recruited to phosphotyrosines in order to attenuate the signaling cascade. One such negative event is phosphotyrosine 992 recruitment of p120 RasGAP, which promotes the intrinsic GTPase activity of Ras and the conversion of Ras-GTP into Ras-GDP. SHP2 is able to suppress this negative regulation by directly dephosphorylating phosphotyrosine 992 , excluding RasGAP binding and therefore promoting Ras activity (49). Similar regulation of RasGAP binding to HER2 phosphotyrosine 1023 has been observed (25).

SHP2 promotes mitogenic signaling through EGFR in other ways, as well. It can directly dephosphorylate the inhibitory Sprouty protein (50), and it can downregulate the activity of the c-Src kinase (Csk), relieving repression of Src after growth factor stimulation (51).

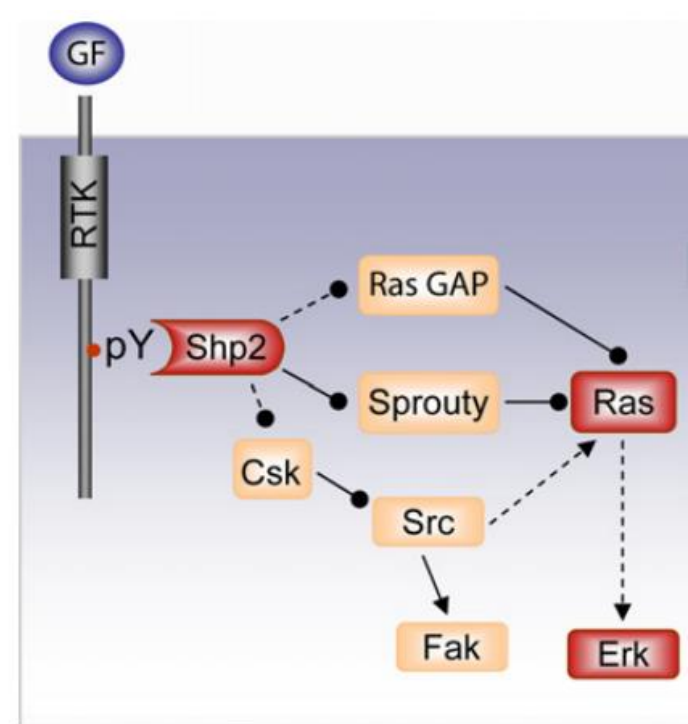

Figure 3. Growth factor signaling can be regulated by SHP2 through the direct dephosphorylation of receptors (e.g., inhibition of RasGAP recruitment) or other mediators (e.g., dephosphorylation of Csk to ultimately activate $\mathrm{Src}$ ) (52).

\subsection{2) SHP2 in cell survival}


In addition to promoting mitogenic signaling, SHP2 is an important player in cell survival (31). This aspect of SHP2 biology has been observed in several growth factor and cytokine signaling contexts. Expression of dominant negative SHP2 abolishes Akt phosphorylation due to either EGF or insulin-like growth factor 1 stimulation, demonstrating its importance in growth factorinduced pro-survival signaling $(45,53)$. Fibroblasts deficient in Akt signaling due to SHP2 inactivation are more susceptible to etoposide-induced apoptosis compared with normal cells (54).

Similar to its promotion of mitogenic signaling, SHP2 action on tyrosine 992 of EGFR or the analogous tyrosine 1023 of HER2 is important for promoting EGF-induced Akt signaling $(25,49)$. Mutation of these residues to phenylalanine enhances Akt activation in response to EGF stimulation. The mechanism of this negative regulation by SHP2 substrate sites has not been fully elucidated, though some studies show a correlation between Ras activation and downstream Akt phosphorylation $(55,56)$.

SHP2 also functions in cytokine-mediated signaling cascades. Cytokines participate in a cascade involving cytokine receptor, which recruits the Janus kinase (JAK) following binding to the cytokine in its extracellular domain. In turn, JAK phosphorylates the receptor, promoting the recruitment of signal transducers and activators of transcription (STATs). JAK is then able to phosphorylate the recruited STAT molecules, stimulating their dimerization. STAT dimers translocate to the nucleus and mediate transcription of target genes. Induction of STAT5, in particular, is regulated by SHP2 in both cell culture following prolactin stimulation (57) and during pregnancy and lactation in mice (58). In addition, interleukin or tumor necrosis factor- 
$\alpha$-induced production of interleukin-6 is blunted in SHP2 -/- fibroblasts, showing that SHP2 can also control upstream cytokine signaling (59).

\subsection{3) SHP2 in cell fate}

Abrogation of SHP2 activity induces embryonic lethality during mid-gestation, in part due to accumulation of undifferentiated cells in the primitive streak, which results in failure to derive the mesodermal tissue lineage (60). This observation is strong evidence in favor of a role for SHP2 in controlling cell differentiation. In another study, embryonic stem cells derived from SHP2-null mice were shown to be less able to differentiate to hemangioblasts in culture $(53,59)$. Conditional deletion of SHP2 in the thymus by driving the expression of Cre recombinase through the Lck promoter inhibited the expansion of CD4+ T-cells, consistent with a defect in Ras/MAPK-related signaling in these cells (61).

\subsection{4) SHP2 in cell motility}

Embryonic lethality in SHP2-null mice is not due only to defective cell differentiation. Defective mesodermal patterning is also observed in these embryos (62). This observation suggests SHP2 is important in gastrulation, similar to the phenomenon observed with focal adhesion kinase or fibronectin knockout animals (62). This suggested that SHP2 played an important role in motility and adhesion signaling. Indeed, embryonic fibroblasts with no SHP2 activity exhibit aberrant focal adhesion formation and condensation of fibrillar actin at the cell periphery. Signaling downstream of FAK and Src following adhesion are inhibited in SHP2-null versus wildtype SHP2 fibroblasts (62). These observations were expanded by analyzing signaling downstream of integrins (63) and growth factors (64), in both cases showing that 
SHP2 controlled the tyrosine phosphorylation of proteins involved in adhesion such as FAK, paxillin, and p130Cas, and the control of cell migration was only partially dependent on enhanced MAPK signaling through SHP2 (64).

Control of cytoskeletal dynamics by SHP2 was first demonstrated in the RhoA pathway by Schoenwelder, et al, suggesting SHP2 signaling results in suppression of RhoA (65). Another study in which SHP2 was artificially partitioned to lipid rafts clarified this role of SHP2. During adherent conditions, activated SHP2 appeared to suppress RhoA signaling; however, when cells were detached, SHP2 promoted RhoA activation (66).

Aside from control of cytoskeletal dynamics, SHP2 has been implicated in the regulation of focal adhesion turnover, an essential process for motility (67). Fibroblasts deficient in SHP2 produce numerous immature focal complexes, independent of RhoA activation. In addition, the dynamics of adhesion proteins was significantly increased in these cells, suggesting SHP2 somehow participates in the maturation and strengthening of focal adhesions.

\section{5) SHP2 in pathology}

Given its importance in normal cell biology, it is not surprising that dysregulation of SHP2 can lead to or enhance disease states. In fact, numerous conditions have been linked to SHP2 hyperactivation or downregulation. This section outlines the phenomena observed during SHP2 dysregulation in humans.

\subsection{1) SHP2 in congenital diseases}

Germline mutations of PTPN11, the gene that encodes SHP2, are prevalent in Noonan syndrome, giving one of the first clues into the significance of SHP2 to human disease. Noonan 
syndrome, characterized by the development of short stature, craniofacial abnormalities, and heart disease, results from missense mutations of SHP2 in more than $50 \%$ of cases (68). These mutations typically affect the interface between the N-SH2 domain and the catalytic domain of the protein, disrupting the interactions and shifting the equilibrium of the protein toward a constitutively open conformation. Noonan syndrome is alternatively caused by missense mutations that enhance the activity of SOS, promoting dysregulated Ras/MAPK signaling (69). This observation and others led researchers to speculate that dysregulated Ras signaling promoted by SHP2 is the primary factor leading to Noonan syndrome in patients.

Paradoxically, LEOPARD (multiple lentigenes, electrocardiographic abnormalities, ocular hypertelorism, pulmonary stenosis, abnormal genitalia, retardation of growth, sensorineural deafness) syndrome is a generally Noonan-like syndrome but with mutations in SHP2 that involve the active site and downregulate its activity (70). The reason for the overlapping symptoms of Noonan and LEOPARD syndromes despite opposite effect on enzymatic activity has not yet been elucidated (71).

\subsection{2) SHP2 in hematopoietic malignancy}

Activating mutations of SHP2 have also been associated with various forms of leukemia, most notably in the case of juvenile myelomonocytic leukemia (JMML). In one study, nearly $35 \%$ of JMML cases demonstrated PTPN11 mutations (72), resulting in enhanced SHP2 activity and signaling downstream of EGF. Some of these mutations result in as high as a 12 -fold induction of SHP2 activation (73). In adult forms of leukemia, SHP2 mutations are rare. Instead, the expression of SHP2 is typically elevated in cancer cells compared with normal bone marrow (74). 
A seminal finding in leukemia was the discovery of the Philadelphia chromosome, a translocation between chromosomes 9 and 22, resulting in a fusion product between the BCR gene and the Abl1 gene that encodes a constitutively-active kinase and leading to chronic myelogenous leukemia (CML) (75). SHP2 has been implicated in transformation by the Bcr/Abl oncogene, since mutation of a Gab2 recruitment site (Y177) inhibits trafficking of Gab2 and subsequent SHP2 and PI3K recruitment and activation (76). This result is in agreement with previous data suggesting the direct association of Bcr/Abl with SHP2, with each protein acting upon the other as substrates (77).

\subsection{3) SHP2 in solid tumors}

The last decade of research into SHP2 has seen a growing appreciation for its role in cancer of different forms $(26,78)$. As in the case of adult leukemias, mutation of SHP2 is quite rare in most solid neoplasia. More often, hyperactivation of the phosphatase is produced by overexpression of SHP2 itself or by overexpression of an activating molecule. An example of the latter phenomenon occurs following stomach infection with the ulcer-causing bacterium Helicobacter pylori. One of its most prominent virulence factors is the CagA protein, a scaffolding molecule that becomes phosphorylated on several tyrosine residues. Phosphorylation promotes the recruitment and activation of SHP2, and this complex enhances gastric carcinoma cell signaling (79).

In lung cancer, a correlation between SHP2 expression and lymph node metastasis has been identified (80). The association of SHP2 with lung cancer is unsurprising due to the predominance of EGFR overexpression or mutation in various forms of the disease, which would lead to constitutive Ras/MAPK signaling that is further enhanced by SHP2 (81). 
Some evidence has come to light in recent years that shows SHP2 can act as a tumor suppressor, depending on cell context. Hepatocellular carcinoma is the first identified disease where clinical data suggest a tumor suppressive function for SHP2. A study of 333 Chinese liver cancer patients found a significant overall survival advantage for patients with elevated SHP2 expression in the tumor compared with normal tissue (82). These results agreed with previous animal-based studies demonstrating a liver-specific deletion of SHP2 enhances inflammatory signaling through STAT3, promoting hyperplastic growth in the liver (83). In all, these results demonstrate that the role of SHP2 in disease is context-dependent, and whether it acts as a tumor suppressor or a proto-oncogene depends on the pathways that are naturally prevalent in the organ or tumor of interest (26).

\subsubsection{1) SHP2 in breast cancer}

The role of SHP2 in breast cancer biology has been studied extensively in the last decade. SHP2 promotes mitogenesis, cell survival, and mesenchymal phenotypes in breast cancer cell culture experiments, and these effects correlate with overexpression of SHP2 protein $(28,84)$. Various mouse models have demonstrated reliance on SHP2 for the maintenance and progression of breast tumors, especially in the context of HER2 dependence (27).

Aside from in vitro phenotype of breast cancer cells, the most important finding regarding SHP2 in breast cancer relates to clinical samples. Three studies have been published exploring this phenomenon. The first of these studies collected 83 invasive ductal carcinoma patient samples and found elevated SHP2 expression levels (assessed by immunohistochemistry) in 73\%; elevated SHP2 levels correlated with both HER2 positivity (79\% of HER2-positive tumors overexpressed SHP2) and lymph node metastasis (37/39 node- 
positive tumors overexpressed SHP2) (28). Aceto, et al noted approximately $91 \%$ of breast tumors showed high expression of SHP2, though they were unable to consistently find differences between normal and tumor expression levels. Still, a greater proportion of patients with a SHP2 gene expression signature (i.e., genes that are downregulated in breast cancer cells when SHP2 expression is inhibited through RNA interference) showed poor prognosis, especially when breast cancer had progressed to invasive ductal carcinoma (27).

The most recent patient study of SHP2 in breast cancer used the highest number of patient samples $(\mathrm{n}=1041)$. Of these, $651(46 \%)$ showed positive expression of SHP2. Significantly, SHP2 positivity correlated with significantly-lower overall survival of patients, leading to a $45.8 \%$ increased risk of mortality over the course of the study (85). In all, these data paint a picture of the importance of SHP2 in progression and maintenance of breast cancer. 


\section{3) HER2 Stabilizes EGFR and Itself by Altering Autophosphorylation \\ Patterns in a Manner That Overcomes Regulatory Mechanisms and \\ Promotes Proliferative and Transformation Signaling}

\section{1) Abstract}

One of the causes of breast cancer is overexpression of the human epidermal growth factor receptor 2 (HER2). Enhanced receptor autophosphorylation and resistance to activation-induced down regulation have been suggested as mechanisms for HER2-induced sustained signaling and cell transformation. However, the molecular mechanisms underlying these possibilities remain incompletely understood. In the current report, we present evidence that show that HER2 overexpression does not lead to receptor hyper-autophosphorylation, but alters patterns in a manner that favors receptor stability and sustained signaling. Specifically, HER2 overexpression blocks EGFR tyrosine phosphorylation on Y1045 and Y1068, the known docking sites of c-Cbl and Grb2, respectively, while promoting phosphorylation on Y1173, the known docking site of the Gab adaptor proteins and phospholipase $\mathrm{C}$ gamma (PLC $\gamma)$. Under these conditions, HER2 itself is phosphorylated on Y1221/1222, with no known role, and on Y1248 that corresponds to Y1173 of EGFR. Interestingly, suppressed EGFR autophosphorylation on the Grb2 and c-Cbl binding sites correlated with receptor stability and sustained signaling, suggesting that HER2 accomplishes these tasks by altering autophosphorylation patterns. In conformity with these findings, mutation of the Grb2 binding site on EGFR (Y1068F-EGFR) conferred resistance to ligand-induced degradation which in turn induced sustained signaling, and increased cell proliferation and transformation. These findings suggest that the Grb2 binding site on EGFR is redundant for signaling, but critical for receptor regulation. On the other hand, mutation of the putative Grb2 binding site in HER2 (Y1139) 
did not affect stability, signaling or transformation, suggesting that Y1139 in HER2 may not serve as a Grb2 binding site. In agreement with the role of EGFR in HER2 signaling, inhibition of EGFR expression reduced HER2-induced anchorage-independent growth and tumorigenesis. These results imply that complementing HER2-targeted therapies with anti-EGFR drugs may be beneficial in HER2-positive breast cancer.

\section{2) Introduction}

The epidermal growth factor receptor (EGFR) family of receptor tyrosine kinases (RTKs) comprises four members that include EGFR1-4. The human counterparts are called HER1-4, also referred to as ErbB1-4 (86-88). All members are composed of an extracellular ligand-binding region, a transmembrane region, and a cytoplasmic region containing a Tyr kinase domain (except HER3) and Tyr autophosphorylation sites. Three of the family members, except HER2 (89), are activated by ligand binding (EGF, TGF $\alpha$, heregulin, amphiregulin and heparin binding EGF) to the extracellular region, while HER2 is a constitutively active protein. Because EGFR1 is commonly known as EGFR and EGFR2 as HER2, we have used these abbreviations throughout this manuscript hereinafter.

The x-ray crystallographic structure of the EGFR ectodomain shows that the dimerization arm is autoinhibited in the resting state by intramolecular interaction between domains 2 and 4 , but EGF binding induces conformational changes that relieve the dimerization arm, leading to homo- or heterodimerization (90-92). On the other hand, no interactions between domains 2 and 4 were observed in the HER2 ectodomain (89). As a result, HER2 can readily heterodimerize with ligandactivated family members or homodimerize with itself, especially under conditions of overexpression, a commonly encountered genetic abnormality particularly in breast cancer (93- 
96). It might be this structural property of HER2 that allows it to act as the preferred partner of heterodimerization with the other family members.

In the EGFR family, ligand activation leads to receptor dimerization and autophosphorylation of tyrosine residues in the C-terminal region that provide docking sites for Src homology 2 (SH2) or phosphotyrosine binding (PTB) domain-containing signaling molecules (97). These interactions lead to recruitment of adaptor proteins such as Grb2, Gab1/2 and Shc that mediate further interactions, translocation of enzymes to substrate micro domains such as PI3K and the Ras nucleotide exchange factor SOS, binding of regulatory proteins such as c-Cbl and RasGAP, and in some instances, activation of enzymes such as SHP1 and SHP2 (98-101). The formation of multiprotein signaling complexes at the level of the receptor accomplishes two major tasks activation of mitogenic and cell survival signaling pathways, and down regulation of receptor activity $(49,102-107)$.

Ligand binding to a receptor tyrosine kinase (RTK) induces endocytosis, ubiquitinylation and degradation, which is considered to be the primary mechanism of regulation. The EGFR family is also regulated by this mechanism (108-111), but HER2 seems to be a poorly regulated RTK even when it is heterodimerized with the other family members $(112,113)$. Although the ability of HER2 to overcome regulatory mechanisms is well recognized, the molecular mechanisms that enable HER2 to accomplish these tasks have remained unexplored. In the current report, we present evidence that show that HER2 overcomes regulatory mechanism by altering autophosphorylation patterns both on itself and the heterodimerized EGFR in a manner that favors downstream signaling and disfavors ligand-induced down regulation. By doing so, HER2 exploits the EGFR to enhance its signaling, transformation, and tumorigenic potency. 


\section{3) Materials and Methods}

\subsection{1) Cells, cell culture and reagents}

The cells used in this study, the MCF10A breast epithelial, and the BT20 and the Skbr-3 breast cancer cell lines, were purchased from ATCC, while the mouse embryo fibroblasts (MEFs) were a

gift from Dr. Steven Frisch (West Virginia University). The MEFs and the BT20 cells were grown in regular Dulbecco's Modified Eagle's Medium (DMEM) supplemented with 10\% fetal calf serum, while the MCF10A were grown in DMEM supplemented with the indicated ingredients $(10 \mu \mathrm{g} / \mathrm{ml}$ recombinant human insulin, $20 \mathrm{ng} / \mathrm{ml}$ EGF (PeproTech), $0.5 \mu \mathrm{g} / \mathrm{ml}$ hydrocortisone, $100 \mathrm{ng} / \mathrm{ml}$ cholera toxin (Sigma) and 5\% horse serum), as described previously (114). All cell lines were grown in a $37^{\circ} \mathrm{C}$ incubator with $5 \% \mathrm{CO}_{2}$ supply. The anti-EGFR polyclonal antibody (E1157), and the anti-FLAG (F3165) and the anti- $\beta$-actin (A5441) monoclonal antibodies were purchased from Sigma-Aldrich, while the anti-HER2 (610162) monoclonal antibody was from Pharmagen. Antibodies to phospho-ERK1/2 (9101S), phospho-Akt (9271S), phospho-EGFR (2234, 2237, 4404, $4407)$ and phospho-HER2 $(2243,2244)$ were from Cell Signaling. The anti-c-CBL antibodies (SC170 and SC-1651) and the anti-panERK2 antibody (SC-81457) were bought from Santa Cruz Biotechnology. Secondary antibodies conjugated with horseradish peroxidase were from Amersham (NA934V and NA931V), and Molecular Probes (A11079). TRITC-labeled EGF was purchased from Molecular Probes.

\subsection{2) Subcloning, retroviral transduction and production of stable cell lines}

Full-length wild type and mutant HER2 and EGFR proteins were subcloned into the REBNA/IRES/GFP retroviral vector as reported previously (115). Recombinant retroviral particles 
expressing the different HER2 and EGFR proteins were produced by transfection into appropriate packaging cells using the FuGENE 6 transfection reagent (Roche). The MCF-10A, the BT20 and the MEF cell lines stably expressing the vector and the different EGFR and HER2 proteins were produced by infection with the respective retroviruses in the presence of $1 \mu \mathrm{g} / \mathrm{ml}$ polybrene (Sigma). After incubation in growth medium for approximately 48 hours, cells were treated with 1 $\mu \mathrm{g} / \mathrm{ml}$ blasticidine, the selection antibiotic expressed by the viral vector, to remove non-expressing cells. Blasticidine-resistant cell populations were used for the various experiments described in this manuscript.

\subsection{3) Stable anti-EGFR shRNA expression}

The expression of the EGFR in the HER2 cells derived from the BT20 line was accomplished as described by us and others (116). Briefly, double-stranded DNA the codes for anti-EGFR shRNA was custom-synthesized (Integrated DNA Technologies) and ligated into the BamHI and EcoRI sites of pSIREN-RetroQ-TetP (BD Biosciences, Palo Alto, CA). The targeting oligonucleotide sequence was 5'GGAGCTGCCCATGAGAAAT-3'. After packaging in appropriate cells, transient supernatants (after 48 hours of incubation) were used to infect the BT20-HER2and the Skbr-3 cells. A non-targeting antiLuciferase shRNA was used as a control. Non-expressing cells were removed by puromycin treatment, and resistant populations were used for the in vivo Xenografting studies described in Fig.7. 


\subsection{4) Preparation of cell lysates, gel electrophoresis and immunostaining analysis}

All cell lysates were prepared in a buffer containing $20 \mathrm{mM}$ Tris- $\mathrm{HCl}, \mathrm{pH} 7.2,150 \mathrm{mM} \mathrm{NaCl}, 50 \mathrm{mM}$

NaF, 1 mM EDTA, 10\% glycerol, 1\% triton-X-100, 1 mM sodium orthovanadate and a protease inhibitor cocktail. Lysates were briefly sonicated to breakdown chromosomal DNA that could interfere with protein separation by polyacrylamide gel electrophoresis. After clearing by centrifugation at 12,000 rpm for 10 minutes, the lysates were analyzed as described in the respective experiments. For separation of proteins, samples were mixed with Laemmli sample buffer, denatured by boiling for 10 minutes, and then run on an $8 \%$ or $10 \%$ denaturing polyacrylamide gel. After transfer onto a nitrocellulose membrane and blocking with 3\% bovine serum albumin, the membranes were stained with primary antibodies overnight at $4^{\circ} \mathrm{C}$, washed 3 times with TBST (Tris-buffered saline containing $0.1 \%$ Tween-20) to remove unbound primary antibody, stained with secondary antibodies for 1 hour at room temperature, washed 3 times with TBST to remove unbound secondary antibody, and finally detected by the chemiluminescence method.

\subsection{5) Immunoprecipitation studies}

Cells expressing vector alone or WT-HER2 were grown to about $80 \%$ density, serum starved overnight, and stimulated with $10 \mathrm{ng} / \mathrm{ml}$ EGF for desired time points. Lysates prepared from these cells were first cleared by centrifugation at $12,000 \mathrm{rpm}$, and then subjected to immunoprecipitation with respective antibodies at $4^{\circ} \mathrm{C}$ overnight. Precipitates were captured on protein $\mathrm{A} / \mathrm{G}$ sepharose beads by further incubation for 1 hour. Finally, precipitates were washed three times with lysis buffer and then eluted by addition of Laemmli sample buffer and boiling for 10 minutes at $100^{\circ} \mathrm{C}$. 
Eluted proteins were separated on polyacrylamide gel electrophoresis and processed for immunostaining as described above.

\subsection{6) Anchorage-independent growth assay}

Anchorage-independent growth in soft agar was performed in $6 \mathrm{~cm}$ cell culture plates. After covering bottom of plates with $0.3 \%$ agar in the growth medium, approximately $10^{5}$ cells suspended in $3 \mathrm{ml}$ of growth medium and mixed with melted agar to a final concentration of $0.3 \%$ were immediately poured onto the agar overlay. The agar cultures were transferred to a $37^{\circ} \mathrm{C}$ and $5 \%$ $\mathrm{CO}_{2}$ incubator and maintained for 15 days. The cells were fed with growth medium containing $0.3 \%$ soft agar twice, and after that, feeding was by replenishing DMEM supplemented with $10 \%$ fetal bovine serum. Colony formation was monitored by visualization under a microscope, and pictures were taken using an Olympus IX71 microscope equipped with DP30BW digital camera.

\subsection{7) TRITC-labeled EGF studies}

Cover slips for TRITC-labeled EGF fluorescence studies were prepared as described previously $(102,117,118)$. Cells grown on cover slips, serum-starved overnight, pre-chilled at $4^{\circ} \mathrm{C}$ for 1 hour, treated with $10 \mathrm{ng} / \mathrm{ml}$ TRITC-labeled EGF for under chilled conditions, washed twice with ice-cold serum-free DMEM, replenished with the same serum-free medium and incubated at $37^{\circ} \mathrm{C}$ for the desired time points. Cover slips were then rinsed with room temperature PBS, fixed in $4 \%$ paraformaldehyde for 20 minutes, washed 3 times with PBS, and mounted on microscopic slides. Pictures were collected using the $40 \times$ objectives of Olympus IX-71. 


\subsection{8) In vivo tumorigenesis studies}

Female nude mice were purchased from Jackson laboratories. Approximately $10^{6}$ MCF-10A-HER2 cells expressing control or anti-EGFR shRNA were mixed 1:1 with matrigel (BD Biosciences) and injected subcutaneously into the rump region of each mouse; three mice were used in each group. Tumor growth was monitored by visual observation. When the control shRNA-HER2 mice developed tumors that were $\geq 1 \mathrm{~cm}$ in diameter, all mice were sacrificed, pictures collected, tumors retrieved and their weights measured.

\section{4) Results}

\subsection{1) HER2 overexpression does not lead to receptor hyperphosphorylation}

HER2 is a constitutively active protein (89), a property that may be key to its oncogenicity. We, therefore, evaluated receptor autophosphorylation patterns under conditions of HER2 overexpression. To address this question, it was necessary to ectopically express HER2 so that the impact of HER2 overexpression could be compared in the same cell line. Due to the significance of HER2 in breast cancer, we have used the non-tumorigenic MCF-10A breast epithelial and the BT20 breast cancer cell lines both of which express a "normal" amount of EGFR and a very low amount of endogenous HER2 and HER3 (119). We have also used the Skbr-3 breast cancer cell line that overexpress HER2, but has a "normal" level of EGFR to see if endogenously expressed EGFR and HER2 function cooperatively in cell transformation. For mechanistic studies involving mutant EGFR and HER2, the mouse embryo fibroblast (MEF) cells were used.

The pattern of EGF-induced total protein tyrosine phosphorylation in the presence and absence of HER2 expression was examined by immunostaining of total cell lysates with anti-pY 
(anti-phosphotyrosine) antibody. To examine the temporal dynamics of tyrosine phosphorylation, time-course EGF stimulation was employed. Surprisingly, the most significant differences were the initial hyperphosphorylation of the EGFR in the controls that rapidly declined after 10 minutes, and the relatively moderate, but sustained receptor autophosphorylation in the HER2 cells (Fig.S1A and B). Reprobing for EGFR showed that it was stabilized in the HER2 cells and rapidly degraded in the controls (Fig.S1A and B), which is consistent with previous reports $(112,113,120)$. Further reprobing with anti-HER2 antibody revealed that HER2 was unaffected by ligand stimulation, confirming our recent report (119). These results suggest that receptor hyperphosphorylation occurs in the absence of HER2, but declines rapidly due to degradation.

The above findings led us concentrate on impact of HER2 overexpression on receptor autophosphorylation. EGFR and heterodimerized HER2 were isolated from cell lysates by immunoprecipitation reactions, and their total phosphorylation state was determined by immunostaining anti-pY antibody. To exclude differences due to ligand-induced receptor down regulation, the first 5 minutes of EGF stimulation was used for these experiments. Input total cell lysates used in these experiments had comparable amount of EGFR in all lanes, and similarly comparable levels of HER2 in the HER2 cells (Fig.1A). Consistent with the results presented in Fig.S1A and B, EGF stimulation induced an enhanced receptor autophosphorylation in the controls, but a relatively modest autophosphorylation in the HER2 cells (Fig.1B). Significant receptor autophosphorylation also occurred in the HER2 cells in the absence of EGF stimulation, suggesting that HER2 can induce basal signaling under conditions of overexpression. Successive reblotting showed that EGFR was efficiently precipitated, and the ectopically expressed HER2 was likewise coprecipitated with EGFR mainly in EGF-stimulation dependent manner. Band density measurements from at least three independent experiments showed that EGF-induced total receptor 
autophosphorylation was lower by approximately 25\% in the HER2 cells (Fig.1C). This looked paradoxical, but it nevertheless provided a clue as to the occurrence of reduced receptor autophosphorylation when HER2 is co-expressed with the EGFR.

\subsection{2) HER2 alters autophosphorylation patterns in a manner that confers differential}

\section{interaction}

The unexpected differences in total receptor autophosphorylation between the controls and the HER2 cells led us investigate the phosphorylation state of individual autophosphorylation sites in both EGFR and HER2 in total cell lysates whose input protein levels are shown in Fig.2A. EGF stimulation led to a robust phosphorylation of the EGFR on Y1045 and Y1068, the docking sites of c-Cbl and Grb2, respectively, in the controls, but phosphorylation on these sites was significantly reduced in the HER2 cells (Fig.2B). In addition, phosphorylation on Y1148, the docking site of the Shc proteins, was lower in the HER2 cells, although not as dramatic as the Grb2 and the c-Cbl sites. On the other hand, phosphorylation on Y1173, the docking site of Gab1 and PLC $\gamma$, was significantly higher in the HER2 cells and lower in the controls. We were also able to determine the state of HER2 autophosphorylation on Y1221/1222, which have no corresponding sites in EGFR, and on Y1248 that corresponds to Y1173 of EGFR. HER2 was phosphorylated even in the absence of EGF stimulation on these sites, which was further enhanced by EGF stimulation. These findings demonstrate that EGFR autophosphorylation is enhanced on the Gab1 (which recruits PI3K and SHP2) and PLC $\gamma$ binding sites, and suppressed on the c-Cbl and the Grb2 binding sites in the presence of HER2. 
The observed differential autophosphorylation pattern could have been due to activation of certain phosphotyrosyl phosphatases (PTPs) by HER2. To verify this point, cells were treated with the general PTP inhibitor orthovanadate and lysates prepared from them were analyzed in the same way as above. Inhibition of PTP activity both in the control and HER2 cells derive from the MCF-10A and BT20 lines did not lead to changes in autophosphorylation patterns (Fig.2C and data not shown). Therefore, the reduced total receptor autophosphorylation in the HER2 cells was due to altered autophosphorylation induced by HER2.

Since altered autophosphorylation might lead to differential interaction with downstream signaling proteins, immunoprecipitation experiments were conducted to test this possibility. Consistent with the autophosphorylation data, the EGF-induced interaction of c-Cbl and Grb2 proteins with EGFR was elevated in the controls and lowered in the HER2 cells (Fig.2D), while the reverse was true for Gab1, p85 (subunit of PI3K) and SHP2. Despite moderate differences in EGFR phosphorylation on Y1148 between the control and the HER2 cells, the interaction of the Shc proteins was significantly lower in the HER2 cells. This outcome might reflect additive effects directly due to reduced pY1148 and indirectly due to reduced pY1068 (Grb2-mediated); note that Shc proteins can make direct and indirect (through Grb2) interactions with activated receptors. Therefore, HER2 promotes altered autophosphorylation that leads to differential interaction with signaling and regulatory proteins. Although we have not directly tested HER2 autophosphorylation at the corresponding Grb2 (Y1139) and c-Cbl (Y1112) binding sites due to lack of specific antibodies, the low level of Grb2 and c-Cbl interaction in the HER2 cells suggests that HER2 may not be phosphorylated on these sites. 


\subsection{3) c-Cbl is unable to interact with the EGFR:HER2 heterodimer, leading to reduced}

\section{ubiquitinylation}

A previous study has shown that controlled EGFR:HER2 heterodimers tend to exclude c-Cbl interaction (121), but the mechanism was not known. The results presented in Figs.2B and C demonstrated that autophosphorylation of the EGFR and possibly HER2 on the c-Cbl and the major Grb2 binding sites is very low in the presence of HER2, eliminating the direct and indirect interaction of c-Cbl. This may be the mechanism by which HER2 excludes c-Cbl. Consistent with this notion EGFR coprecipitated with c-Cbl efficiently in the controls, and poorly in the HER2 cells

(Fig.3A and B). Furthermore, HER2 was undetectable in c-Cbl immunoprecipitates, confirming that c-Cbl does not interact with EGFR-HER2 heterodimers. Reprobing with anti-c-Cbl antibody showed that the amount of c-Cbl was comparable in all lanes. These results, together with the findings in Fig.2B and D, show that the mechanism by which HER2 excludes the interaction of c-Cbl is through suppression of autophosphorylation on the direct and indirect c-Cbl binding sites.

Based on the results in Fig.3A and B, it was reasoned that exclusion of c-Cbl interaction by HER2 could lead to inhibition of ubiquitinylation. Consistent with this assumption, EGFR in the control cells was highly ubiquitinylated, but less so in the HER2 cells despite the presence of comparable amount of receptor molecules in all lanes (Fig.3C and D). Therefore, the mechanism by

which HER2 protects itself as well as EGFR from ligand-induced ubiquitinylation and degradation is by regulating the interaction of c-Cbl through altering autophosphorylation patterns. 


\subsection{4) HER2-induced inhibition of Grb2-binding site autophosphorylation does not}

\section{perturb signaling}

Inhibition of EGFR autophosphorylation on Y1068 in the HER2 cells was unexpected as this site was previously reported to promote Ras activation by acting as the major Grb2-SOS complex docking site. We therefore tested the state of EGF-induced downstream signaling under our experimental conditions. Because HER2 is known to induce sustained signaling, these experiments were conducted in a time course fashion. ERK1/2 and Akt activation was sustained in the HER2 cells and short-lived in the controls, suggesting the existence of an inverse relationship with elevated phosphorylation of the Grb2 binding site in EGFR (Fig.4A and D). In agreement with the dynamics of EGF-induced EGFR degradation (Fig.S1 and (119)), initial ERK1/2 and Akt activation

(first 10 minutes) was comparable in both the controls and the HER2 cells, but rapidly declined in the former and sustained in the latter. It was also possible to discern a revamping pattern in ERK1/2 and Akt activation in the HER2 cells after 2 hours, reflecting receptor recycling as reported by us recently (119). As compared to the controls, the HER2 cells showed basal ERK1/2 and Akt activation, suggesting that HER2 alone can induce a low level of constitutive signaling. AntipanERK2 immunostaining showed that the amount of total protein loaded to each lane was comparable. Band density measurements (Fig.4B, C, E and F) confirmed that ERK1/2 and Akt activation in both cells were comparable at the 10 minutes time point, but rapidly declined in the controls to approximately $20 \%$ of the initial value, and never fell below $50 \%$ of the initial value in the HER2 cells. Therefore, the low level of Grb2 binding-site autophosphorylation in the presence of HER2 does not perturb signaling. 


\subsection{5) Y1068F-EGFR induces sustained signaling compared to the wild-type counterpart}

The induction of sustained signaling by HER2 while suppressing Grb2 binding required direct testing. Site-directed mutagenesis was used to replace Y1068 in EGFR and Y1139 in HER2 with Phe, which were referred to as Y1068F-EGFR and Y1139F-HER2, respectively. The vector, the wild-type and the mutant proteins (FLAG-tagged at the c-terminus) were expressed by retrovirus transduction in the mouse embryo fibroblast (MEF) cells that have very low-to-undetectable level of endogenous EGFR and HER2. Time-course EGF stimulation studies were conducted to examine the impact of the Grb2 binding-site mutation on the signaling and dynamics of ligand-induced receptor regulation. Expression of both WT-EGFR and Y1068F-EGFR led to an enhanced ERK1/2 and Akt activation initially, but it was short lived in the WT-EGFR and sustained in the Y1068FEGFR cells (Fig.5A). Anti-EGFR immunostaining revealed that Y1068F-EGFR was resistant to EGFinduced degradation, but the wild type counterpart was not. With regard to HER2, both WT-HER2 and Y1139F-HER2 were able to induce sustained ERK1/2 and Akt activation, which was slightly enhanced by EGF stimulation (Fig.5B). Reprobing for HER2 showed that both the WT-HER2 and the Y1139F-HER2 proteins were unaffected by EGF stimulation. Hence, Y1139, the putative Grb2 binding site in HER2, does not seem to play any significant role either in mediating signaling or receptor down regulation.

The above results led us determine the state of Grb2, SOS and c-Cbl interaction with the wildtype and the mutant EGFR proteins by immunoprecipitation experiments. As expected, mutation of the Grb2 binding site significantly reduced the interaction of Grb2 and SOS with EGFR (Fig.5C). The interaction of c-Cbl was very low in the Y1068F-EGFR cells regardless of the presence of intact Y1045, suggesting that the Grb2-mediated (indirect) interaction plays a major role in recruiting cCbl to EGFR. Consistent with loss of c-Cbl interaction, Y1068F-EGFR was less ubiquitinylated than 
the wild type counterpart. Reprobing for EGFR showed that comparable amount of both WT-EGFR and Y1068F-EGFR were present in all lanes. These findings suggest that Grb2 mediates receptor ubiquitinylation and degradation by acting as an adaptor to c-Cbl. In support of our conclusion, previous reports have also shown that Grb2 mediates receptor degradation by recruiting c-Cbl $(122,123)$. Therefore, HER2-induced suppression of EGFR autophosphorylation at the Grb2 binding site protects EGFR from ligand-induced degradation, and affords the EGFR with the ability to induce sustained signaling.

\subsection{6) EGF-induced Y1068F-EGFR trafficking resembles that of WT-EGFR in presence of}

\section{HER2}

The resistance of Y1068F-EGFR to ligand-induced degradation was very similar to that induced by HER2 coexpression (Fig.S1A and B, (119)). To further compare this possibility, the dynamics of ligand-induced EGFR trafficking was studied by fluorescence microscopy in MEF cells ectopically expressing WT-EGFR or Y1068F-EGFR, and in BT20 and in MCF-10A cells expressing vector alone or HER2. Cells were treated with TRITC-EGF at $4^{\circ} \mathrm{C}$ to initiate binding without inducing internalization, hence allowing a synchronized endocytosis and processing when cells are transferred back to $37^{\circ} \mathrm{C}$ after removal of unbound EGF (118). Both WT-EGFR and Y1068F-EGFR expressed in MEFs were at the plasma membrane when cells were fixed immediately, but incubation at $37^{\circ} \mathrm{C}$ led to internalization within 10 minutes (Fig.5D). Similarly, EGFR in both the control and the HER2 cells derived from the BT20 and the MCF-10A lines was at the plasma membrane at the zero time point, but was readily internalized within 10 minutes of incubation at $37^{\circ} \mathrm{C}$ as depicted by speckled cytoplasmic distribution (Fig.5E and F). Upon further incubation, 
ectopically expressed WT-EGFR in the MEFs, and endogenous EGFR in the control BT20 and MCF$10 \mathrm{~A}$ cells was sorted to the perinuclear region and decayed gradually. On the other hand, Y1068FEGFR in the MEFs behaved like WT-EGFR in the HER2 cells; in these cells, most of the EGFR signal was sorted to one side of the nucleus with gradual outward extension and minimal decline. Therefore, the dynamics of EGF-induced Y1068F-EGFR processing resembles HER2 overexpression-induced EGFR recycling.

\subsection{7) Y1068F-EGFR is more transforming when compared to the wild-type counterpart}

The results presented in Fig.5 demonstrated that the Y1068F-EGFR is more signaling competent than the wild type counterpart, while both the wild type and the mutant HER2 proteins are equally competent. The biological significance of these observations was further tested by evaluating changes in cell morphology, growth and transformation. In the absence of EGF stimulation, neither the wild-type nor the mutant EGFR protein induced any appreciable change in cell morphology, proliferation or transformation (data not shown). In the presence of EGF ( $2 \mathrm{ng} / \mathrm{ml}$ ), however, the distinctive properties of the two proteins became apparent. MEFs expressing the Y1068F-EGFR acquired an elongated and refractive morphology in 2D (Fig.S2A), an increased proliferative potential (Fig.6A, left) and ability to form colonies in soft agar (Fig.6B). On the other hand, the mere expression of both WT-HER2 and Y1139F-HER2 proteins induced morphological changes characterized by an interspersed and refractive appearance that forms foci-like structures de novo (Fig.S2B). In addition, both HER2 proteins were capable of inducing increased cell proliferation (Fig.6A, right), and robust colony formation in soft agar (Fig.6C). These results suggest that Y1068F-EGFR is superior in inducing cell growth and transformation than its wild type 
counterpart, which in turn suggests that inhibition of EGFR phosphorylation on Y1068 by HER2 confers signaling and transformation efficiency. Furthermore, these data show that mutation of Y1139 in HER2, the putative Grb2-binding autophosphorylation site, does not perturb transforming ability, suggesting that Y1139 in HER2 may not play any significant role. Overall, the superiority of HER2 in inducing enhanced cell growth and transformation suggests that it has additional effects other than altering autophosphorylation patterns.

To further corroborate the role of EGFR in HER2-induced transformation, its expression was silenced in the Skbr-3 breast cancer cell line that naturally has amplified HER2 gene and normal level of EGFR. Silencing EGFR in these cells reduced the size of HER2-induced colony growth in 3D without significantly affecting colony number (Fig.6D-F). These results confirm the ectopic HER2 overexpression data in the BT20 and the MCF-10A cells that EGFR contributes significantly to HER2-induced signaling and transformation.

\subsection{8) HER2 exploits the EGFR to enhance its tumorigenic potential}

The results so far described demonstrate that HER2 alters autophosphorylation patterns to confer resistance to ligand-induced degradation and to ultimately enhance cooperative signaling and transformational capacity. Finally, we sought to test whether or not the normally-expressed EGFR contributes significantly to HER2-iduced tumorigenesis in vivo.

The BT20 HER2 cells expressing control or anti-EGFR shRNA were injected subcutaneously into nude mice, and tumor formation was monitored by visual observation. The HER2 cells in which EGFR expression was silenced formed smaller and rounded tumors, while those that co-expressed EGFR with HER2 formed larger and irregular ones (Fig.6G). Pictures of isolated tumors further showed differences in tumor size and shape (Fig.6H). Measuring tumor weight confirmed that 
blocking EGFR expression in the HER2 cells reduced tumor burden by approximately 3.5 fold (Fig.6I). Therefore, HER2-induced tumorigenesis is dependent on EGFR, suggesting that the normally-expressed EGFR may potentiate the oncogenic property of HER2 in HER2-positive breast cancer. In other words, HER2 is capable of mustering signaling efficiency from the normally expressed family members by promoting their stability through altering autophosphorylation patterns.

\section{5) Discussion}

HER2 is an oncogenic transmembrane tyrosine kinase that induces cell transformation and tumorigenesis when it is overexpressed in tissues. Its overexpression, primarily due to gene amplification, is one of the major causes of breast cancer. HER2 is a constitutively active protein (89) with intrinsically low tyrosine kinase activity $(124,125)$. This property of HER2 may be responsible for its ability to escape activation-induced down regulation, one of the suggested mechanisms for its oncogenesis. We thus investigated the effect of HER2 overexpression on receptor autophosphorylation and its impact on protein stability, downstream signaling, and cell transformation. Furthermore, we have presented data that show that EGFR contributes significantly to HER2-induced tumorigenesis in xenograft mice models.

We have shown that HER2 overexpression leads neither to increased overall cellular tyrosine phosphorylation nor to receptor hyper-autophosphorylation. To the contrary, receptor hyperautophosphorylation occurs in the absence of HER2 (Fig.1B and C). These data were indicative of the occurrence of reduced receptor autophosphorylation when EGFR and HER2 heterodimerize. Analyzing specific autophosphorylation sites demonstrated that phosphorylation of the EGFR on Y1045 and Y1068 (the docking sites of c-Cbl and Grb2, respectively) was suppressed in the absence 
of HER2 while the opposite was true for Y1173 of EGFR and for Y1221/1222 and Y1248 of HER2 (Fig.2B). Although we have not determined phosphorylation of the putative c-Cbl and Grb2 docking sites (Y1112 and Y1139, respectively) in HER2 due to lack of specific antibodies, the modest overall autophosphorylation suggests that phosphorylation on these sites may not occur.

In agreement with the autophosphorylation data, c-Cbl and Grb2 interact strongly with EGFR homodimers, and poorly with EGFR:HER2 heterodimers (Fig.2D). The poor interaction of c-Cbl and Grb2 with HER2-containing complexes provides further evidence that HER2 may not be phosphorylated on Y1112 and Y1139. The low level of c-Cbl binding-site autophosphorylation in the presence of HER2 lends mechanistic support to the previous observation that EGFR:HER2 complexes tend exclude c-Cbl binding (121). In addition to its signaling role, Grb2 is known to mediate the interaction of c-Cbl with activated receptor molecules (123). Hence, the low level of Y1068 phosphorylation in the presence of HER2 blocks the indirect interaction of c-Cbl (Fig.2D, and Fig.3A and B), which in turn leads to the low level of receptor ubiquitinylation (Fig.3C and D). Therefore, the mechanism by which HER2 confers receptor stability is through altered autophosphorylation. However, our results cannot exclude the existence of other as yet unidentified ubiquitin ligases excluded by HER2 that may contribute to receptor resistance to ligand-induced degradation.

While blocking autophosphorylation on the c-Cbl and the Grb2 binding sites, HER2 promotes EGFR phosphorylation on Y1173 and its own phosphorylation on Y1248 and Y1221/1222 (Fig.2B). Phosphorylated Y1173 of EGFR and Y1248 of HER2 are known docking sites for the Gab1/Gab2 adaptor proteins $(126,127)$. Consistent with the phosphorylation data, the interaction of Gab1 was elevated in the HER2 cells, and lowered in the control cells (Fig.2D). Upon binding to RTKs and 
phosphorylation on multiple tyrosine residues, Gab1 serves as a docking platform for several signaling proteins, leading to formation of multiprotein complexes. Because EGFR and HER2 lack tyrosine residues that mediate direct PI3K binding, the interaction of PI3K with the EGFR:HER2 complexes is most probably through Gab1. Previous reports suggest that SHP2, the known positive effector of EGFR and HER2 signaling, interacts with EGFR and HER2 through Gab1 $(128,129)$. Therefore, the increased interaction of SHP2 with EGFR and HER2 complexes may as well be through Gab1.

HER2 does not significantly influence the state of EGFR phosphorylation on Y1148, the docking site of the Shc adaptor proteins, but relatively less Shc proteins bind to EGFR:HER2 heterodimers. The reduced Shc interaction may be related to low Grb2 binding in the HER2 cells; note that Shc proteins are known to also make indirect interaction with RTKs through Grb2(130,131). Hence, the low level of EGFR autophosphorylation on Y1068 in the presence of HER2 reduces the interaction of Grb2 directly and that of c-Cbl and Shc proteins indirectly. A previous phosphoproteomic study suggested that Y1222 in HER2 may mediate Shc binding (132), but our results demonstrate very low binding of Shc proteins regardless of enhanced HER2 phosporylation on Y1221/1222. It is possible that HER2 molecules behave differently inside (this report) and outside (132) the cell. Nonetheless, the presence of HER2 autophosphorylation on these sites suggests that they may contribute to the direct and indirect binding of other signaling proteins. It will be interesting to address these points in future studies. Overall, our results show that HER2 alters autophosphorylation patterns of the EGFR and of itself, favoring Gab1, p85 (PI3K) and SHP2, and disfavoring c-Cbl and Grb2 interactions. 
The mechanism by which HER2 alters the autophosphorylation pattern within the heterodimer is unknown at this stage. Recent structural and modeling studies on receptor tyrosine kinases suggest that the kinase domains of dimerized receptor molecules form asymmetric dimmer that has a positive allosteric effect on kinase activation $(133,134)$. This has been demonstrated for EGFR homodimers, FGFR1 homodimers, FGFR2 homodimers, and HER2 and HER4 heterodimers. The occurrence of such kinase domain asymmetry in the EGFR:HER2 heterodimer has not been demonstrated, but it is thought to be a common phenomenon (135). It is possible that subtle structural arrangements in the EGFR:HER2 heterodimer hinder phosphorylation on the direct and indirect c-Cbl interaction sites, while promoting on those sites that mediate downstream signaling. A second possibility is that the kinase domain of HER2 may have the ability to discriminate among autophosphorylation sites, leading to distinctive autophosphorylation. A third possibility might be that the intrinsically low tyrosine kinase activity of HER2 $(124,125)$ may lead to phosphorylation of some, but not all sites on EGFR and HER2 itself, leading to differential autophosphorylation patterns. Future structural studies on EGFR:HER2 heterodimers may be needed to delineate between these possibilities.

Because HER2 promotes the interaction of the SHP2 tyrosine phosphatase, the known positive effector of RTK signaling, including EGFR and HER2, one may also argue that HER2 suppresses autophosphorylation on the c-Cbl and Grb2 binding sites indirectly by facilitating SHP2-mediated dephosphorylation. However, this possibility is unlikely since SHP2 is a highly selective phosphatase, targeting only RasGAP docking sites in EGFR (49) and HER2 (25). The involvement of other PTPs also is unlikely since treatment with orthovanadate, the general PTP inhibitor, did not lead to differences in the autophosphorylation patterns (Fig.2C). In addition, the occurrence of this 
event in less than 2 minutes of EGF stimulation strengthens the notion that HER2 does not act through tyrosine phosphatases to modulate autophosphorylation patterns.

One of the most unexpected findings was the induction of sustained signaling by the EGFR mutant lacking the Grb2 binding site (Y1068F-EGFR) (Fig.5A). Analysis of EGF-induced receptor regulation has revealed that $\mathrm{Y} 1068 \mathrm{~F}-\mathrm{EGFR}$ is relatively resistant to degradation. Immunoprecipitation studies further revealed that EGFR lacking the Grb2 binding site interacts poorly with c-Cbl and is least ubiquitinylated (Fig.5C), explaining the observed resistance to ligandinduced degradation. A further layer of evidence came from fluorescent-tagged EGF stimulation studies which demonstrated that Y1068-EGFR is efficiently internalized upon EGF stimulation, but not degraded. Hence, the sustained signaling efficiency of the Y1068F-EGFR is related to its resistance to EGF-induced degradation. Comparison of ligand-induced Y1068F-EGFR sorting with HER2-induced sorting of endogenous EGFR (compare Fig.5D with E and F) showed a striking similarity, providing experimental evidence for the HER2-induced suppression of EGFR autophosphorylation on Y1068 conferring resistance to ligand-induced degradation. The increased cell proliferation and transformation induced by the Y1068F-EGFR (Fig.6A and B) provide further support for this role of HER2.

The commonly-held notion is that Grb2 mediates Ras activation by acting as an adaptor to the nucleotide exchange factor SOS to bind to activated receptor tyrosine kinases at the plasma membrane where functional Ras resides. The finding that HER2 efficiently activates the Ras-ERK signaling pathway while suppressing Grb2 interaction contrasts with this notion. The ability of the Y1068F-EGFR to induce enhanced and sustained ERK1/2 and Akt activation, while having significantly reduced Grb2-SOS binding confirms the current findings. The corresponding HER2 
mutant (Y1139F-HER2) that lacks Grb2 binding site also signals as efficiently as the wild-type counterpart. Therefore, interaction of SOS with activated RTKs through Grb2 to activate Ras may be redundant or dispensable. In fact, the current data suggest that the major role of Grb2 bound to pY1068-EGFR is to mediate a receptor regulatory loop by coordinating the interaction of c-Cbl. A recent study suggested that the ERM proteins (ezrin, radixin, moesin) may play critical role in recruiting SOS to Ras (136), which may also be functional in HER2 signaling. Future studies are needed to further elucidate how HER2 activates the Ras-ERK signaling pathway without utilizing Grb2.

Conferring receptor resistance to activation-induced down regulation through modulation of receptor autophosphorylation patterns seems to be the mechanism for HER2 in promoting sustained signaling and cell transformation. The results presented in the current report (Fig.6D-I) reveal that maximal transformation and tumorigenesis by HER2 was dependent on EGFR. Loss of EGFR expression abolished HER2-induced anchorage-independent growth by the HER2 amplified breast cancer cell line. Furthermore, the enhanced tumor growth induced by HER2 overexpression in the BT20 breast cancer cell line was significantly suppressed, suggesting that HER2 exploits the normally expressed EGFR to maximize its oncogenicity. Based on ${ }^{3} \mathrm{H}$-thymidine-incorporation experiments (137), it was previously suggested that EGFR is not required for HER2-induced cell proliferation which is in contrast to our findings. It may be necessary to determine the significance of EGFR in HER2-induced transformation using transformation and in vivo studies in the future. Nonetheless, the implication of our findings is that complementing HER2-targeted therapies with anti-EGFR drugs may be beneficial in HER2-positive breast cancer as also evidenced by recent clinical trials where the dual tyrosine kinase inhibitor lapatinib was demonstrated to provide 
clinically beneficial results when administered in combination with anti-HER2 drugs such as herceptin (138).

\section{6) Acknowledgement}

This work was supported by a grant number CA124940 from the National Cancer Institute (NCI), a component of the National Institute of Health (NIH) to YMA. The authors do not have any commercial affiliation or conflict of interest to disclose.

\section{7) Figure Legends}

Figure 1: Analysis of EGF-induced receptor autophosphorylation. The control and the HER2 cells derived from the MCF-10A and the BT20 lines were serum starved overnight and then stimulated with EGF (10 ng/ml) for 2 minutes, 5 minutes or left unstimulated. A) Input total cell lysate analysis for EGFR and HER2 in the MCF-10A breast epithelial and in the BT20 breast cancer cell lines. HER2 was expressed in both cells by retrovirus-mediated transduction. The expression of EGFR in all lanes and HER2 in the corresponding lanes was comparable. B) EGFR was immunoprecipitated from the total cell lysates shown in A, and then analyzed for total autophosphorylation by immunostaining with anti-pY antibody. Reblotting with anti-EGFR and anti-HER2 antibodies showed that EGFR was successfully precipitated, and HER2 was likewise co-precipitated in the expected lanes. C) Band density measurement of anti-pY blots of EGFR in the controls, and EGFR and HER2 heterodimers in the HER2 cells. The results shown were mean \pm S.D. (standard deviation) taken from three independent experiments.

Figure 2: Analysis of specific autophosphorylation and interaction. The control and the HER2 cells were serum starved overnight and then stimulated with EGF $(10 \mathrm{ng} / \mathrm{ml})$ for the indicated time 
points. A) Input total cell lysate analysis for EGFR, HER2, Gab1, c-Cbl, p85 (PI3K), SHP2, Shc, Grb2 and $\beta$-actin (loading control). B) Analysis of individual autophosphorylation sites in EGFR and HER2 using phospho-specific antibodies. In this study, four EGFR and three HER2 autophosphorylation sites were analyzed. C) Effect of PTP inhibition on receptor autophosphorylation patter. Cells were treated with $1 \mathrm{mM}$ orthovanadate for 30 minutes prior to EGF stimulation. Lysates prepared from these cells were analyzed for EGFR and HER2 autophosphorylation as shown. D) Immunoprecipitation and immunostaining analysis of c-Cbl, Grb2, Shc, Gab1, p85 (PI3K) and SHP2 interaction with EGFR and EGFR:HER2. Note that the interaction of Grb2 and Shc was higher in the control cells and lower in the HER2 cells, and the opposite was true for Gab1, p85 (PI3K) and SHP2.

Figure 3: Analysis of EGFR and HER2 coprecipitation with c-Cbl in the MCF-10A (A) and BT20 (B) cell lines. The control and the HER2 cells were serum starved overnight and then stimulated with EGF $(10 \mathrm{ng} / \mathrm{ml})$ for the indicated time points. Lysates prepared from these cells were subjected to anti-Cbl immunoprecipitation, and then to immunostaining with the indicated antibodies. State of receptor ubiquitinylation (top), HER2and EGFR coprecipitation (middle and bottom, respectively) were analyzed by immunostaining with specific antibodies. Data from the MCF-10A is shown in C while from the BT20 in D.

Figure 4: Impact of HER2 expression on signaling. As compared to the controls, expression of HER2 led to an enhanced and sustained ERK1/2 and Akt activation in both the MCF-10A (A) and BT20 (D) lines. Band density analysis of ERK1/2 and Akt activation in the MCF-10A- (B and C) and BT20- (E 
and F) derived lines showed short-lived signaling in the controls and sustained signaling with slight decline and revamp in the HER2 cells. Basal pERK1/2 and pAkt activation levels (no EGF stimulation) in the controls were used as reference to calculate "fold over basal". The results shown are averages of three independent experiments \pm S.D. (standard deviation).

Figure 5: Mutation of the Grb2 binding site in EGFR and HER2 does not perturb signaling. A) Vector, WT-EGFR or Y1068F-EGFR were expressed in the mouse embryo fibroblast (MEF) cells by retroviral transduction, and their impact on EGF-induced ERK1/2 and Akt activation was analyzed by immunostaining with specific antibodies. Anti-EGFR immunostaining showed that the expression of both EGFR proteins was comparable. Note also that Y1068F-EGFR is resistant to EGFinduced degradation. B) Similarly, the vector and the WT-HER2 and the Y1139F-HER2 proteins were expressed in MEFs and analyzed for ERK1/2 and Akt activation and HER2 expression. Note that both WT-HER2 and Y1139F-HER2 induce constitutive signaling, and their stability was unaffected by EGF stimulation. C) Interaction of SOS, Grb2 and c-Cbl with EGFR proteins and state EGFR ubiquitinylation were analyzed by immunoprecipitation with anti-FLAG and immunostaining with the indicated antibodies. D) Dynamics of EGF-induced WT-EGFR and Y1068F-EGFR processing in MEFs. Also shown in E and $\mathrm{F}$ are dynamics of EGF-induced EGFR (endogenous) processing in the MCF-10A and BT20 lines, respectively. For fluorescence studies, cells were serum-starved overnight, chilled at $4^{\circ} \mathrm{C}$ for 1 hour, treated with $10 \mathrm{ng} / \mathrm{ml} \mathrm{TRITC-labeled} \mathrm{EGF} \mathrm{at} 4^{\circ} \mathrm{C}$ for 1 hour, transferred to $37^{\circ} \mathrm{C}$ and incubated for the indicated time points. Preparation of coverslips and picture collection was as described in the materials and methods. 
Figure 6: A) Impact of EGFR (left) and HER2 (right) expression on cell proliferation was determined by counting cells in 10 random $4 \mathrm{x}$ objective fields and then averaging. The results shown are mean \pm S.D. (standard deviation) of three independent experiments. B) Expression of Y1068F-EGFR, but not WT-EGFR in MEFs induced colony formation in soft agar in the presence of 2 ng/ml EGF. C) Expression of both WT-HER2 and Y1139F-HER2 in MEFs induced robust colony formation in soft agar. D) Pictures of colonies, one at $10 \times$ and another at $5 \times$ objectives for each group, showing effect of EGFR silencing on colony formation by the Skbr-3 HER2-positive breast cells. Scale bar represents $500 \mu \mathrm{m}$. E) Bar graph showing differences in colony size formed by the control and EGFR-shRNA cells after 10 days of incubation in soft agar. F) Bar graph showing colony number as determined by counting 10 random fields under $4 \times$ objective and then averaging; data shown is mean \pm S.D. (standard deviation) of three independent experiments. G) Pictures of mice bearing xenograft tumors initiated by subcutaneous injection of $\sim 10^{6}$ BT20-HER2 cells expressing control or EGFR-specific shRNA. Note the differences in size and shape where the shRNA are smaller and relatively rounded while the controls are larger and irregular. $\mathrm{H}$ ) Pictures of isolated tumors also show differences in size and shape. I) Bar graph showing differences in tumor weight between the control and the shRNA cells; data shown is average weight of the three tumors in each group \pm S.D. (standard deviation).

Figure S1: Expression of HER2 in the MCF-10A (A) and BT20 (B) cell lines did not lead to increased total protein tyrosine phosphorylation. In fact, HER2 expression led to reduced tyrosine phosphorylation as at the level of EGFR and HER2 which suggests reduced receptor 
autophosphorylation.. Note that EGFR in the HER2 cells is resistant to EGF-induced degradation, and HER2 itself was unaffected by EGF stimulation in both the MCF-10A and BT20 derived cells.

Figure S2: A) Expression WT-EGFR in MEF cells and addition of $2 \mathrm{ng} / \mathrm{ml} \mathrm{EGF}$ in the growth medium did not lead to any appreciable morphological changes, while expression of Y1068F-EGFR under same conditions resulted in morphological changes reminiscent of cell transformation. Note that the Y1068F-EGFR cells form foci-like cellular mass, suggesting loss of contact-induced growth inhibition. B) Expression of both WT-HER2 and Y1139F-HER2 led to transformation of MEF cells as evidenced by refractive appearance and formation of foci-like cellular mass. The foci-like structures are suggestive of absence of contact-induced growth inhibition. 
Figure 1. Analysis of EGF-induced receptor autophosphorylation

a Input total cell lysate
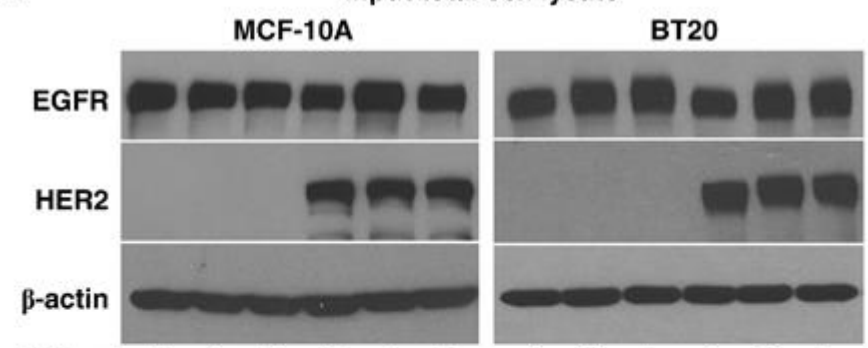

EGF, $\min \frac{025}{\mathrm{CON}} \frac{0}{\mathrm{HER} 2} \quad \frac{2}{\mathrm{CON}} \frac{0}{\text { HER2 }}$

b

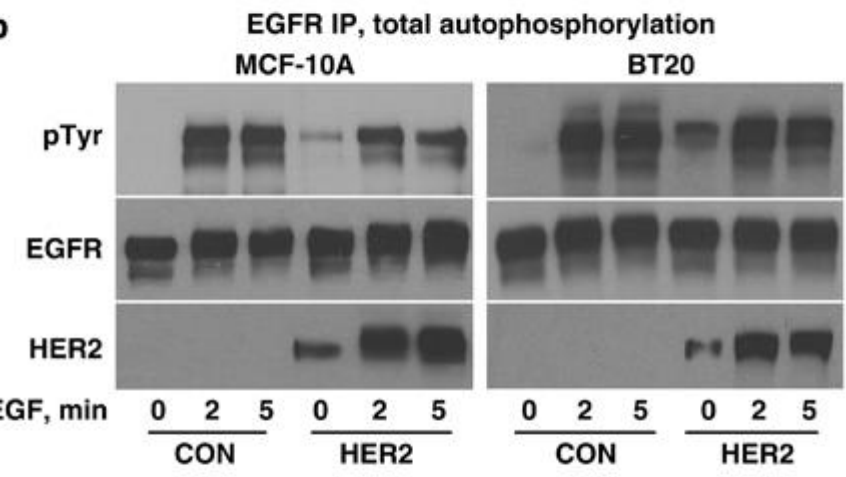

c Band density
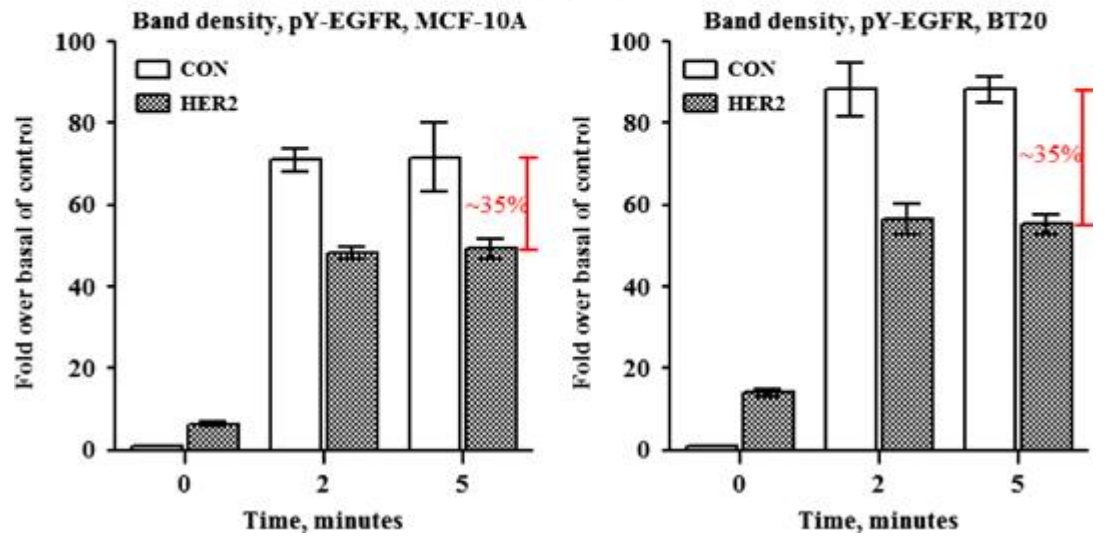
Figure 2. Analysis of specific autophosphorylation and interaction.

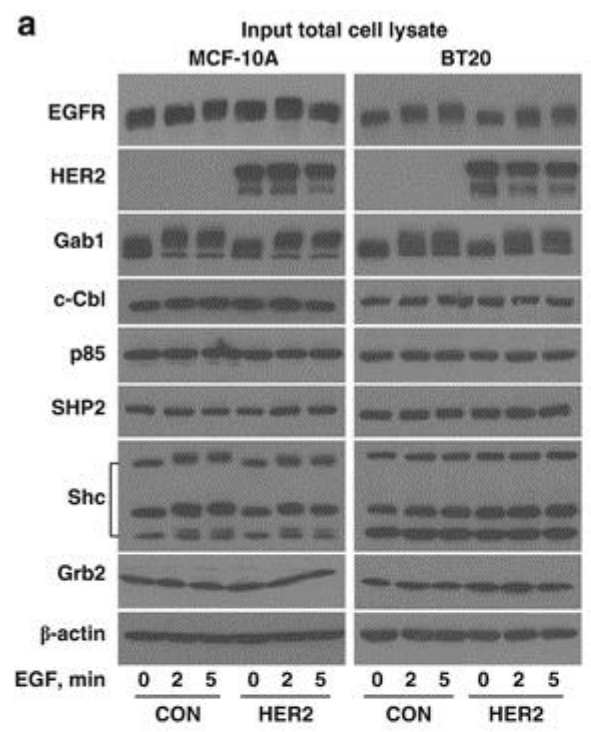

b HER2 promotes selective autophosphorylation
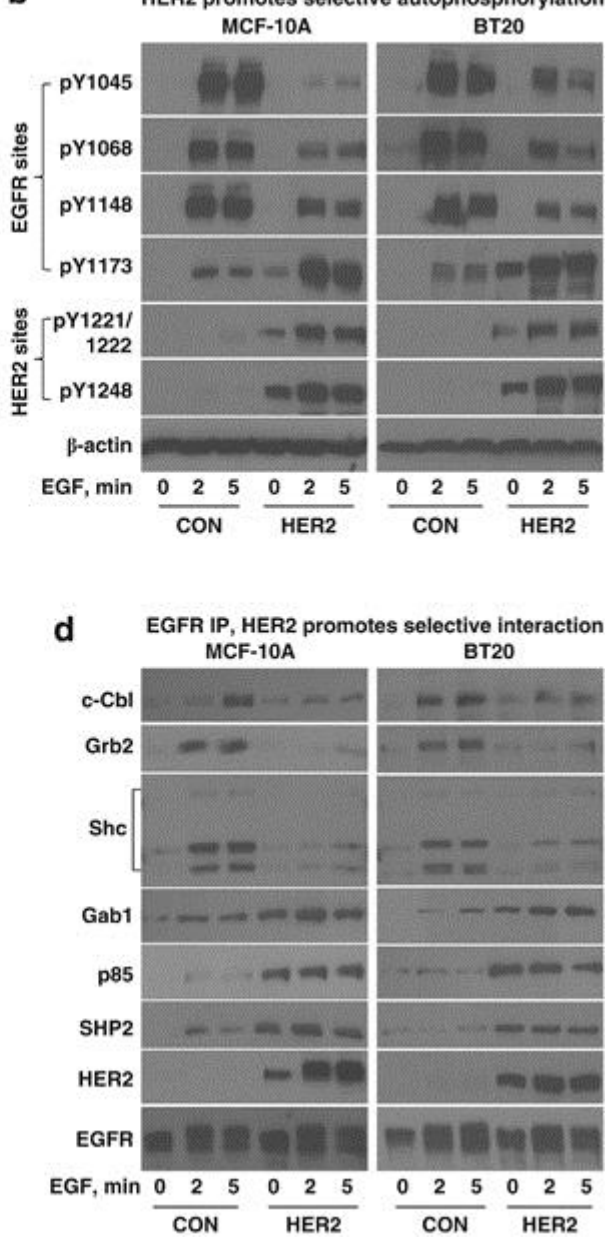
Figure 3. Analysis of EGFR and HER2 co-precipitation with c-Cbl
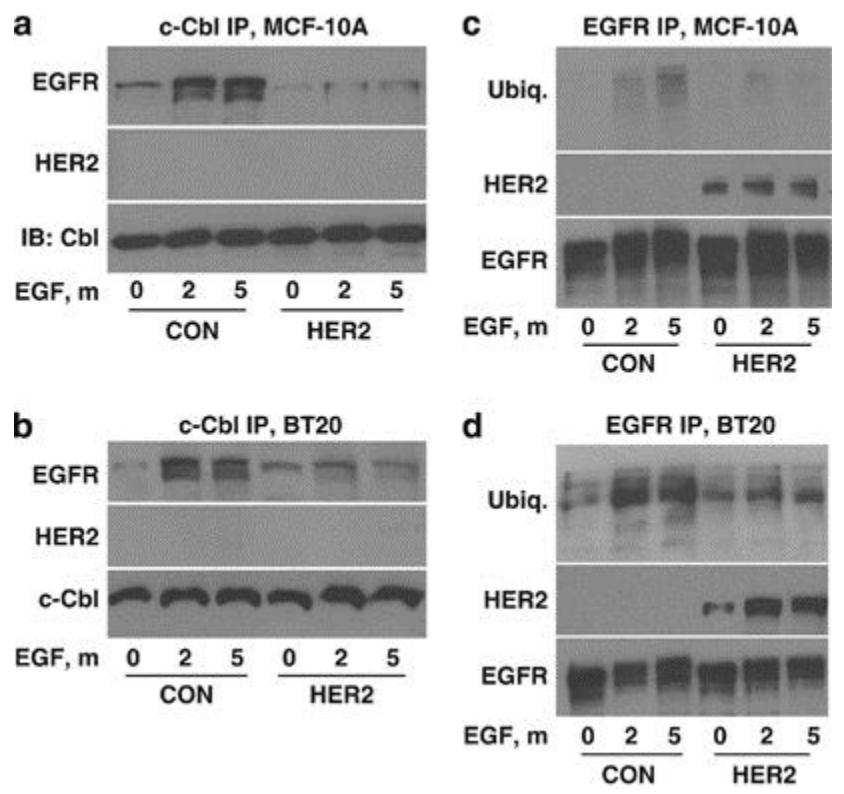
Figure 4. Impact of HER2 expression on signaling

a
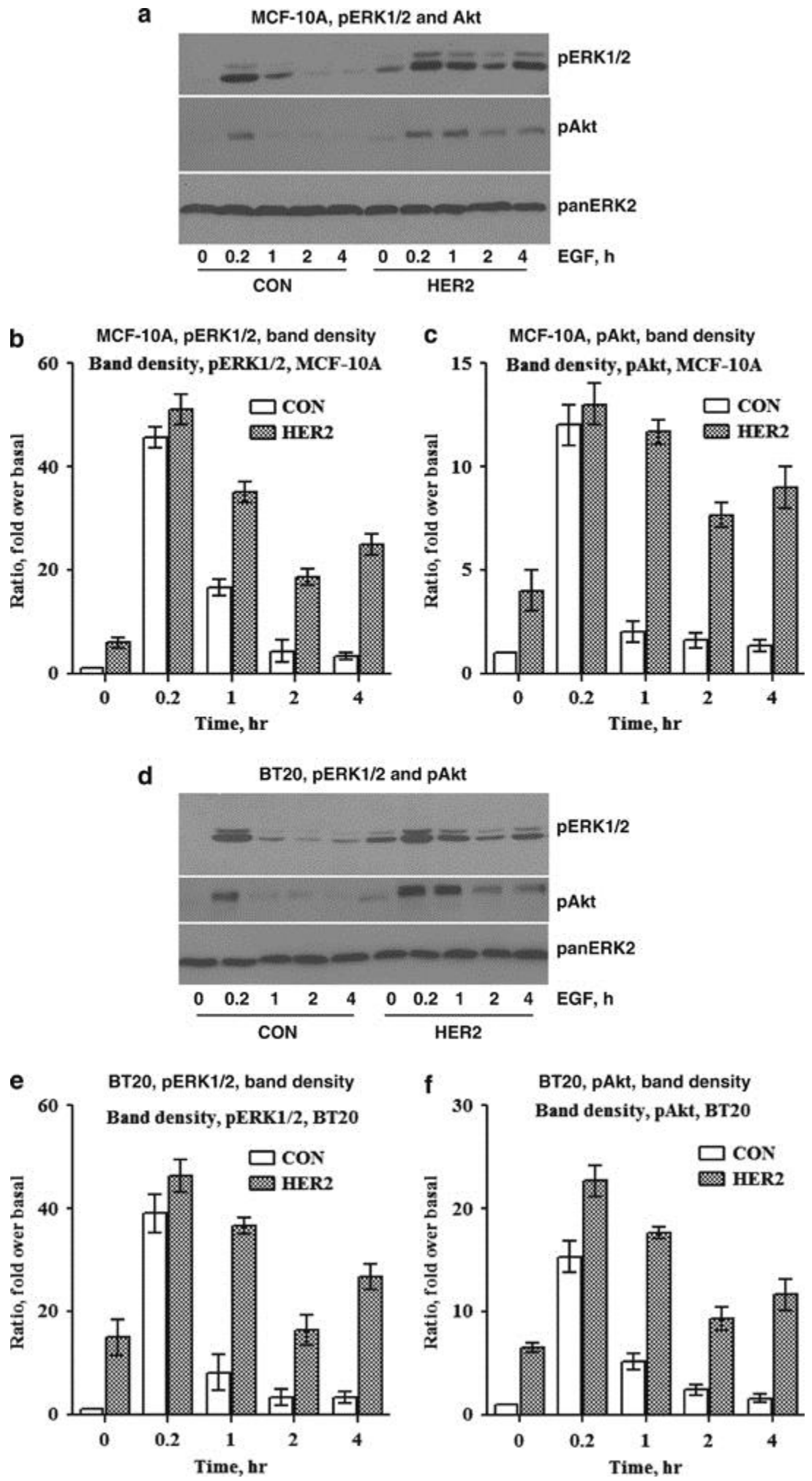
Figure 5. Mutation of the Grb2-binding site in EGFR and HER2 does not perturb signaling

a

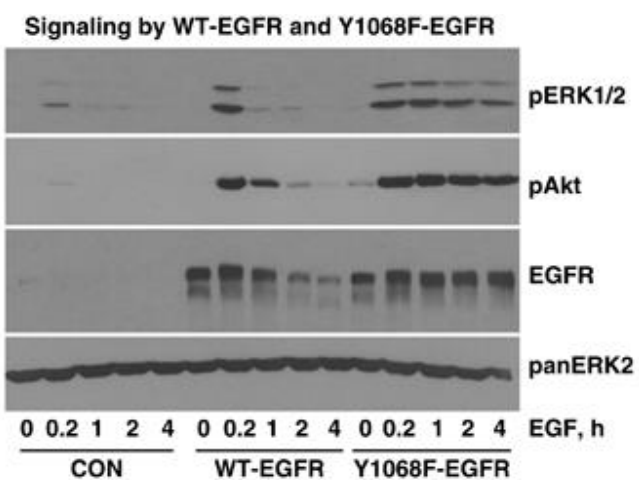

b

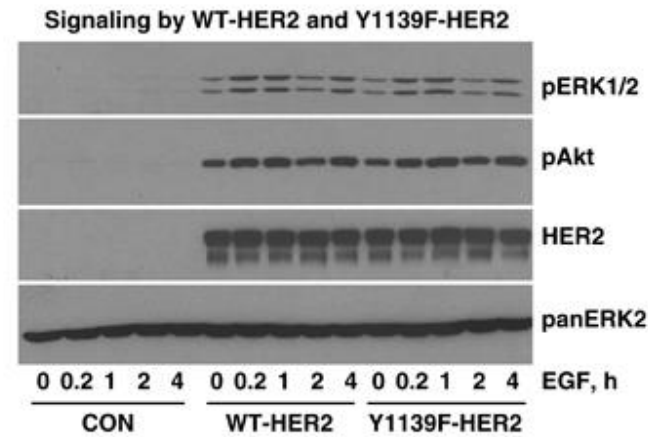

c

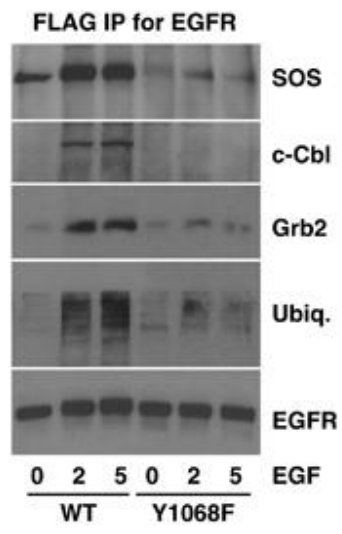

e MCF-10A, TRITC-EGF fluorescence, $40 x$ objective
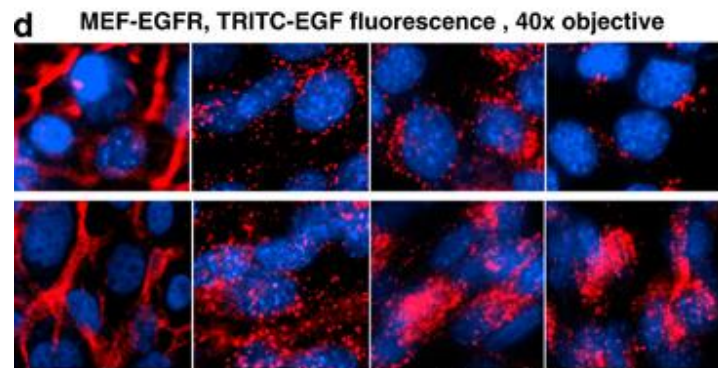

WTEGFR

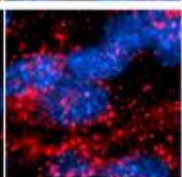

$10 \mathrm{~min}$
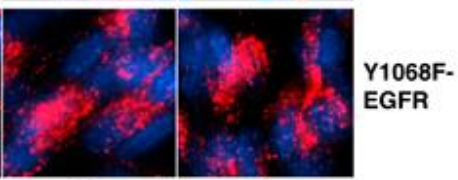

1h

$2 \mathrm{~h}$

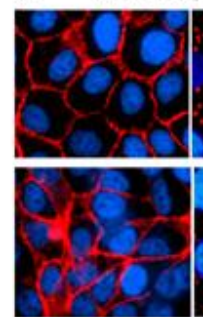

0

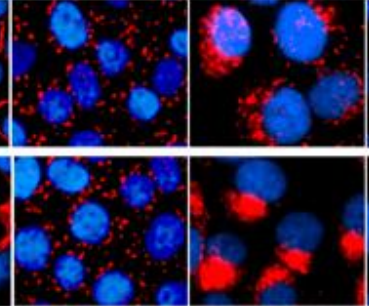

$10 \mathrm{~min}$

$1 \mathrm{~h}$

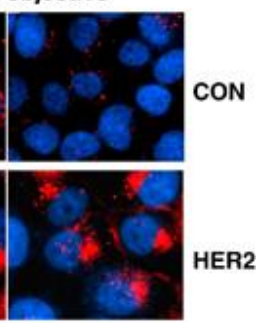

$2 \mathrm{~h}$

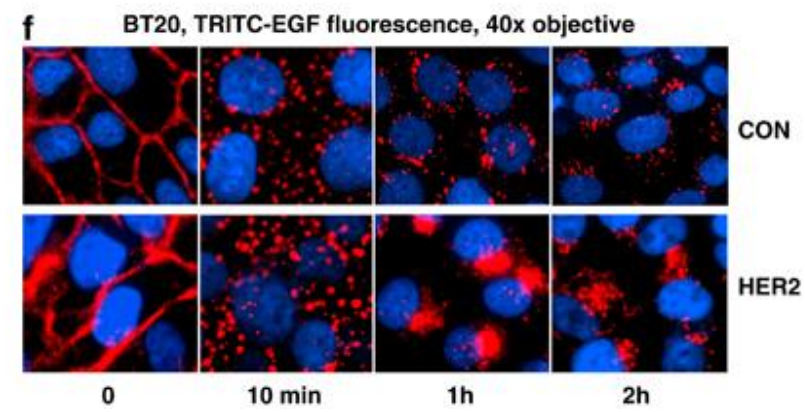


Figure 6. Impact of EGFR mutation on HER2-induced transformation and tumorigenesis
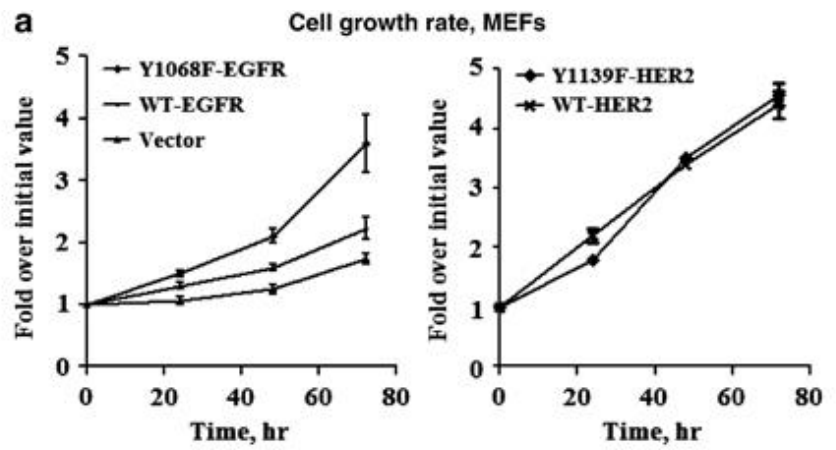

b

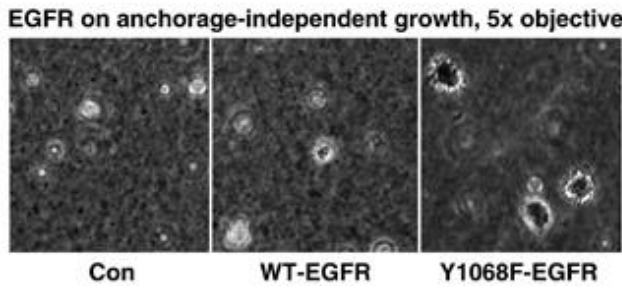

c

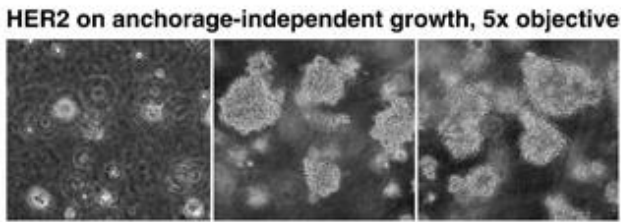

Con d
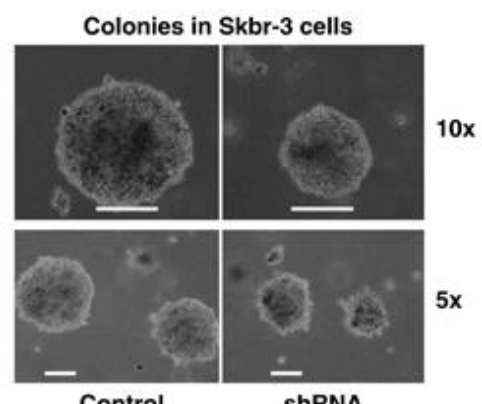

Control

Xenograft tumor growth in nude mice

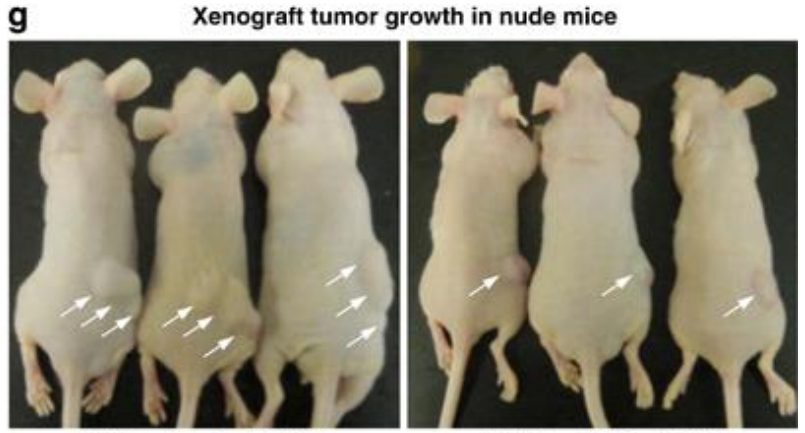

HER2 + EGFR ShRNA h

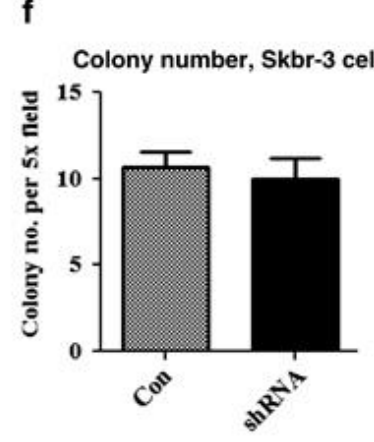

(1)

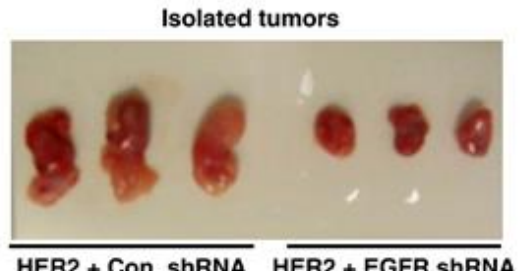

HER2 + Control ShRNA

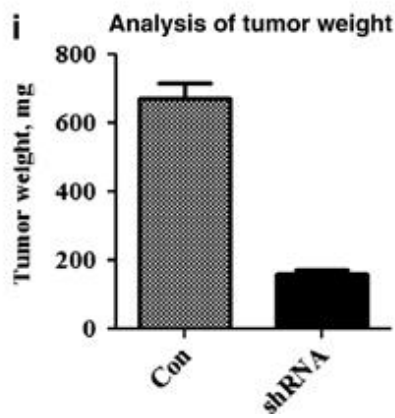

Figure S1A. 
A) MCF-10A cells

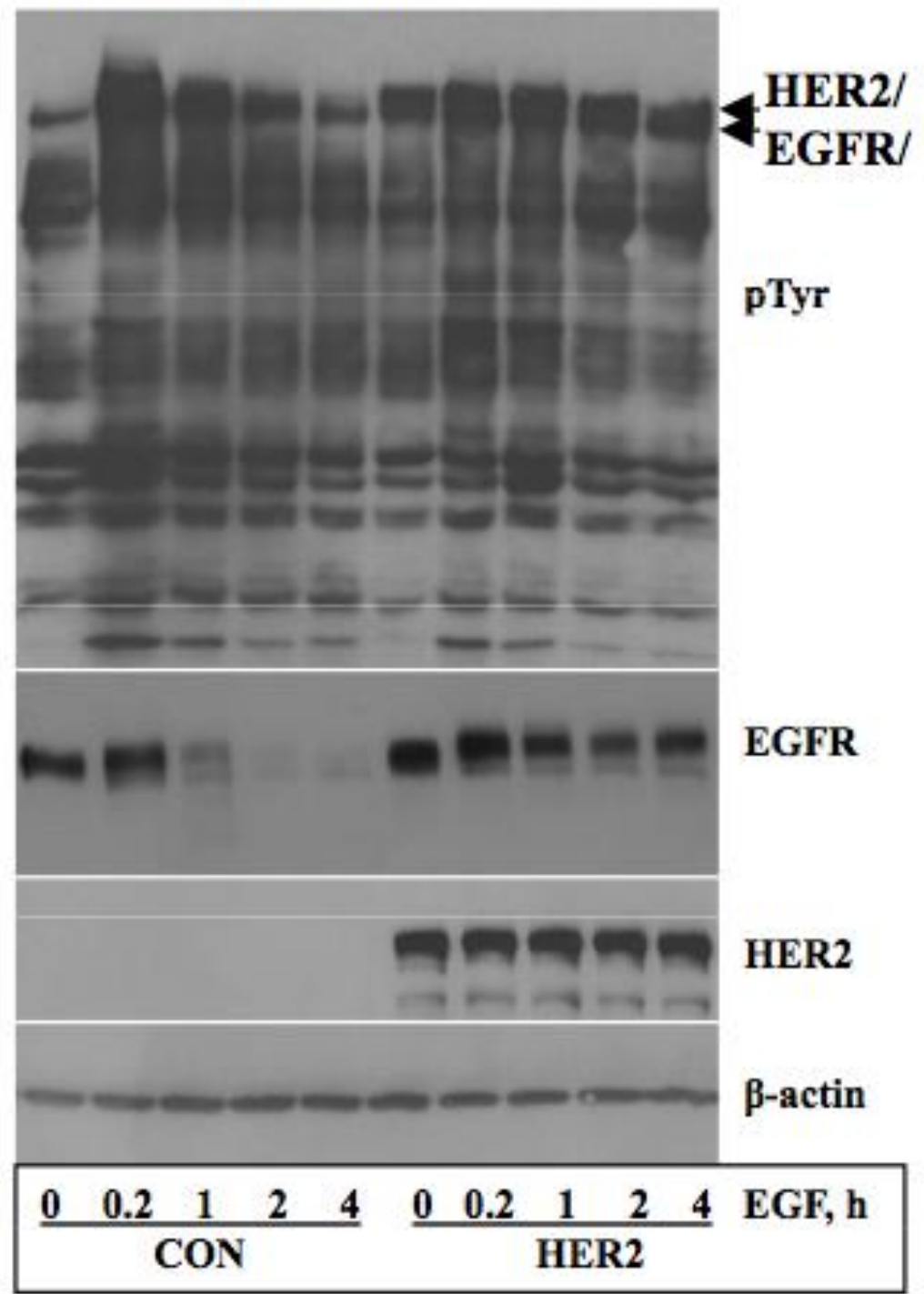


Figure S1B.

B) BT20 cells

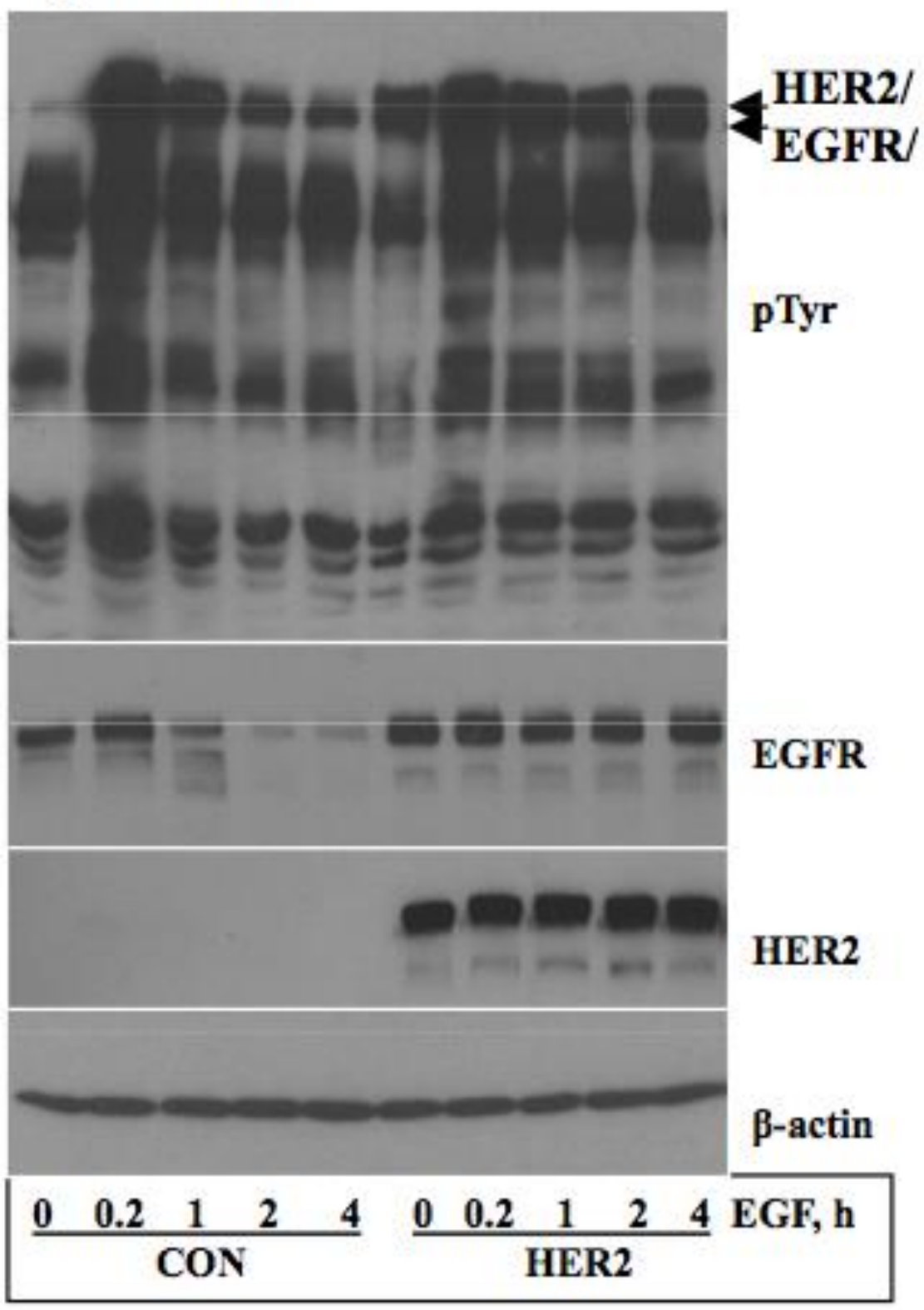


Figure S2.

A) Transformation by EGFR, $5 X$ objective

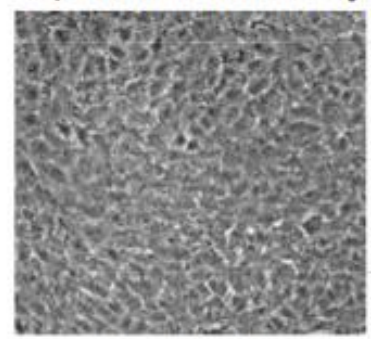

Con+EGF

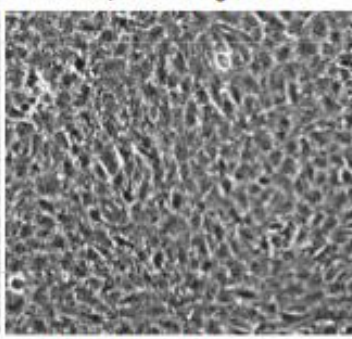

WT-EGFR+EGF

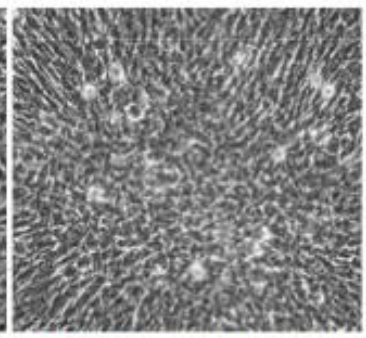

Y1068F-EGFR+EGF

B) Transformation by HER2, $5 X$ objective

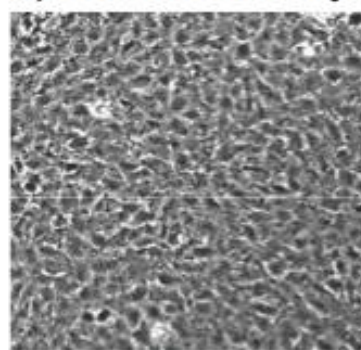

Con

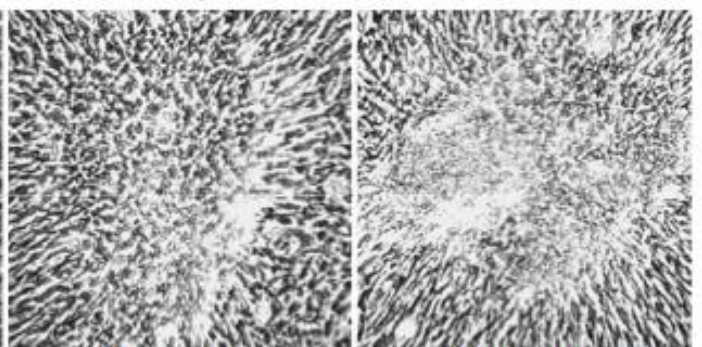

WT-HER2

Y1139F-HER2 


\section{4) The tyrosine phosphatase SHP2 regulates focal adhesion kinase to promote EGF-induced lamellipodia persistence and cell migration}

Zachary R. Hartman ${ }^{1}$, Michael D. Schaller ${ }^{1}$ and Yehenew M. Agazie ${ }^{1,2}$, Department of

Biochemistry ${ }^{1}$ and The Mary Babb Randolph Cancer Center ${ }^{2}$, School of Medicine, West Virginia University, Morgantown, WV 26506 


\section{1) Abstract}

The Src homology 2-containing protein tyrosine phosphatase (SHP2) is very well known for its positive role in cell growth and survival signaling induced by receptor tyrosine kinases (RTKs), including the epidermal growth factor family of RTKs. Furthermore, SHP2 is known to promote cell migration and invasiveness, key steps in cancer metastasis, in a variety of cell types, including breast cancer cells. To date, however, the mechanism by which this phosphatase regulates cell movement has not been fully elucidated. In the current report, a new role for SHP2 in controlling cell migration has been suggested. We show that SHP2 mediates lamellipodia persistence and cell polarity to promote directional cell migration in the MDAMB-231 and the MDA-MB-468 basal-like and triple-negative breast cancer cell lines. We further show that SHP2 modulates the activity of focal adhesion kinase (FAK) by dephosphorylating pTyr397, the autophosphorylation site that primes FAK function. Because hyperactivation of FAK is known to weaken nascent focal complexes and hence negatively regulate their maturation to focal adhesions, we propose that one of the mechanisms by which SHP2 promotes lamellipodia persistence is by downregulating FAK activity through dephosphorylation of pTyr397. The finding that inhibition of FAK activity partially restores epidermal growth factor-induced cell migration under conditions of SHP2 inhibition supports our proposition SHP2 promotes growth-factor-induced cell movement by acting, at least in part, on FAK. FAK inhibition did not rescue migration defects in SHP2-silenced cells under nonstimulated conditions, however, suggesting that SHP2 also acts through other mechanisms. 


\section{2) Introduction}

The Src homology 2-containing phosphotyrosylphosphatase 2 (SHP2) is a cytosolic enzyme that catalyzes the hydrolysis of phosphate from tyrosine residues(139,140). As its name indicates, SHP2 contains two tandemSH2 domains in the N-terminal region and a phosphatase domain in the C-terminal region. In addition, SHP2 contains sites of tyrosine phosphorylation in the C-terminal tail region (140). Structural studies have shown that the N-terminal SH2 domain interacts with the catalytic site, resulting in autoinhibition by obstructing access to substrates. Engagement of this domain with phosphotyrosine results in conformational changes that relieve the active site (39). Therefore, factors that can upregulate cellular phosphotyrosine such as exposure of cells to receptor tyrosine kinase (RTK) ligands (141), overexpression or activating mutations of receptor tyrosine kinases, expression of the CagA protein in H. pylori infection (142), activation of integrins during matrix adhesion (143), and cytokine stimulation (144) can lead activation of SHP2. In addition, mutations within the inhibitory SH2 domains of SHP2 can abolish autoinhibition, leading to constitutive activation $(70,145)$. In fact, such mutations do occur in human diseases and are demonstrated to be the causes for disorders such as Noonan syndrome and associated cardiac disorders and hematopoietic malignancies (146).

SHP2 typically acts as a positive mediator in a variety of signaling pathways (147). For example, SHP2 mediates EGF-induced signals to the Ras-extracellular signal-regulated kinases 1 and $2($ ERK1/2) and the PI3K (phosphatidylinositol-3) - Akt (protein kinase B) pathways by counteracting RasGAP (Ras GTPase activating protein), an inducer of Ras inactivation. It does so, at least in part, by dephosphorylating RasGAP-docking sites on membrane proteins such as the epidermal growth factor receptor (EGFR) (49) and the human EGFR2 (HER2 also known as 
ErbB2) (25) thereby blocking recruitment the plasma membrane where functional Ras resides. In addition, SHP2 has been shown to promote RTK signaling by inactivating Sprouty proteins which are responsible for receptor downregulation (148), and to enhance Src activation by dephosphorylating PAG (phosphoprotein associated with glycosphingolipid-enriched microdomains) which is known to act as a docking site for Csk, an inhibitor of Src (149). This positive effect on signaling has been shown to promote cell transformation and tumorigenesis. For instance, SHP2 is essential for cell transformation induced by the constitutively active fibroblast growth factor receptor 3 (150),the oncogenic form of Src (v-Src) (151) and the HER2oncogene (25). Recently, SHP2 was shown to be important for xenograft tumor growth of breast cancer cells (152).The discovery that the SHP2 protein is overexpressed in breast cancer (28) further strengthened the notion that SHP2 plays critical roles in breast cancer and possibly in other cancers where tyrosine kinase signaling is the driving force.

SHP2 has been shown to regulate cell migration in a variety of ways $(63,153,154)$, a process that is known to promote cancer metastasis. For instance, SHP2 modulates focal adhesion dynamics $(64,67)$, RhoA activity (155), and integrin signaling (63) which are all implicated in regulating cell migration. To date, however, the mechanism by which SHP2 controls cell migration has not been fully understood. Because SHP2 overexpression is strongly correlated to breast cancer metastasis (28), we sought to explore its role in cell migration in basal-like and triple-negative breast cancer (BTBC) cell lines, which are known to be highly invasive. In this report we show that SHP2 promotes cell migration by mediating directional cell polarization and lamellipodia persistence, which are key steps in this process. We further show that SHP2 regulates the activity of focal adhesion kinase (FAK) through 
dephosphorylating pTyr397 (pY397), the autophosphorylation site that controls the kinase activity, to promote cell migration.

\section{3) Materials and Methods}

\subsection{1) Cells and reagents}

Cells used in this study included wild type and focal adhesion kinase-null murine embryonic fibroblasts (MEFs) kindly provided by Dr. Steven Frisch (West Virginia University) and the MDA-MB-231 and the MDA-MB-468 BTBC cell lines purchased from ATCC. All cells were grown in Dulbecco's modified eagle medium supplemented with $10 \%$ fetal bovine serum at 37 ${ }^{\circ} \mathrm{C}$ and $5 \%$ carbon dioxide. The polyclonal (cat.\# SC-280) and the monoclonal (cat.\# SC-7384) anti-SHP2 antibodies were from Santa Cruz, the FLAG-tag (cat. \#F1504) and anti- $\beta$-actin antibodies (cat \#A5441) were from Sigma-Aldrich, focal adhesion kinase (cat \#F15020), phospho-Y397-FAK (cat \#F25420) and anti-GM130 (cat. \#610823) were from BD biosciences, anti-cortactin antibody (clone 4F11) was a kind gift from Dr. Scott Weed (West Virginia University), and anti-paxillin antibody (cat \#05-417) was from Upstate Biotechnology. The FAK inhibitor (PF-573228) was purchased from Pfizer.

\subsection{2) Silencing SHP2 expression with shRNA}

Double-stranded deoxyoligonucleotides that code for short hairpin ribonucleotides that target SHP2 mRNA were custom- synthesized (Integrated DNA Technology) and ligated into the BamHI and EcoRI sites of a lentivirus backbone termed pLSL-Puro (a gift from Dr. Peter Chumskoy, Cleveland Clinic Foundation) in a manner similar to Ivanov, et al (156). We used the targeting sequences 5'-GTATTACATGGAACATCAC-3' and 5'-GAAGAATCCTATGGTGGAA-3' which were previously shown to be SHP2 specific (157). The shRNA construct and supporting 
plasmids (pCMV-SVV-G and sPax2) were transected into 293T packaging cells using the turbofect transfection reagent (Fermentas). After $48 \mathrm{~h}$, supernatants containing viral particles were collected, filtered using $0.22 \mu \mathrm{m}$ syringe filter and used for infecting the BTBC cell lines. Polybrene at concentration of $3 \mu \mathrm{g} / \mathrm{ml}$ was added to enhance the infection efficiency. After 48 hours, cells were treated with $4 \mu \mathrm{g} / \mathrm{ml}$ puromycin to remove non-infected ones. A short hairpin against luciferase was used as a control in these studies.

\subsection{3) Site-directed mutagenesis, subcloning and expression}

FAK cDNA in a pCMV-SPORT6 vector, purchased from ATCC, was used as the template for sitedirected mutagenesis using a Stratagene kit (cat.\# 600250). The Y397F sense and anti-sense primers were 5'-TCAGAAACAGATTTTGCTGAGAT and 5'-ATCTATAATCTCAGCAAAATCATCTG, and the Y407F sense and anti-sense primers were 5'-GATTATAGATGAAGAAGATACTTTCA and 5'-CTGGTTGAGGGCATGGTGAAAGTATC. The wild-type and the mutant FAK cDNA were then subcloned into the viral vector reported previously (115) at XhoI and NotI sites using the forward primer containing a Sall site (5'-TTTGTCGACCGCCACCATCGCAGCT-3'), and a reverse primer containing a NotI site (5'-TTTTGCGGCCGCCTATTTATCATCATCAT-3').Subcloning FLAGtagged SHP2 into a retroviral vector and expression in target cells was described previously (25). Retroviral particle production, infection of target cells, selection and production of population of cells stably expressing FAK proteins were also performed as described for SHP2 proteins previously (25). Populations of cells stably expressing SHP2 or FAK proteins were used for the various experiments described in the relevant sections. 


\subsection{4) Preparation of cell lysates, immunoprecipitation and immunostaining}

Cells were lysed in a buffer containing $20 \mathrm{mM}$ Tris- $\mathrm{HCl}$ (pH 7.4), $150 \mathrm{mM} \mathrm{NaCl}, 1 \mathrm{mM}$ EDTA, 1\% Triton-X-100, 10\% glycerol, and $50 \mathrm{mM} \mathrm{NaF}$ supplemented with $10 \mu \mathrm{g} / \mathrm{ml}$ each of aprotinin, leupeptin, and PMSF for protease inhibition, and 10mM sodium orthovanadate for inhibition of phosphatases. For immunoprecipitation, the lysates were cleared by centrifugation at 12,000 rotations per minute, incubated overnight with a primary antibody, and then precipitated using protein $\mathrm{G}$ sepharose beads. The beads were washed 3 times with cell lysis buffer and denatured by boiling with Laemmli sample buffer. The same denaturation procedure was used for analyzing total cell lysates. Denatured proteins were separated on $8 \%$ or $10 \%$ polyacrylamide gel by electrophoresis, immobilized onto a nitrocellulose membrane, blocked with 3\% bovine serum albumin in Tris-buffered saline containing 1\% Tween-20 (TBST), and stained with a primary antibody overnight at $4{ }^{\circ} \mathrm{C}$. Next, membranes were washed 3 times with TBST and then incubated with a horseradish peroxidase-conjugated secondary antibody in 5\% milk. The immunostains were visualized by the enhanced chemiluminescence method (Pierce Inc.).

\subsection{5) Monolayer wound-healing assay}

The MDA-MB-231 and the MDA-MB-468 cells (expressing control shRNA or SHP2 shRNA) were grown to confluency in $6 \mathrm{~cm}$ dishes. A scratch was made using a P-200 pipette tip, and cell migration was monitored and pictures collected under an Olympus IX-71 microscope equipped with a CCD camera. For EGF-stimulated migration, cells nearing confluence were serum starved overnight, wounds made in the same manner as above, re-fed with serum-free media, and then treated with $100 \mathrm{ng} / \mathrm{ml}$ EGF for 2 hours or left untreated. The Olympus Microsuite software was used to estimate the cell-free area of the wounds. The change in the 
wound width was estimated by dividing the area with the constant length of the images taken at $4 \mathrm{x}$ objective similar to that reported by Tao, et al (158).

\subsection{6) Immunofluorescence}

Cells were seeded on fibronectin-coated coverslips and grown for the indicated time points described in the respective figure legends. They were then washed twice with roomtemperature PBS, fixed in 3.5\% paraformaldehyde in PBS for 20 minutes, permeabilized with 0.6\% Triton X-100 in PBS for 30 minutes, incubated in 3\% bovine serum albumin (BSA) in PBS for 1 hour at room temperature, and incubated with primary antibodies overnight at $4{ }^{\circ} \mathrm{C}$. Alexafluor 488-conjugated rabbit and Alexafluor 568-conjugated mouse secondary antibodies were used to detect antigens. Images were captured using an Olympus IX71 microscope with attached CCD camera and Microsuite Basic Edition software.

\subsection{7) Assay for golgi orientation}

Cells were seeded on fibronectin-coated coverslips and grown to confluency after which monolayer wounds were made by scratching with P-200 pipette tip. The cells were allowed to close the wound for approximately 6 hours and were then processed for immunofluorescence staining with anti-GM-130 antibody (golgi marker) as described above. 10 fields of fluorescent pictures per slide were obtained at 10x magnification and were analyzed for golgi orientation as described previously (159). At least 1000 cells per slide were counted in this way.

\subsection{8) Live-cell imaging and kymographic analysis}

Cells expressing either control shRNA or SHP2 shRNA were transiently transfected with $3 \mu \mathrm{g}$ of mCherry-actin (Clontech) using the FuGene 6 transfection reagent (Roche), incubated overnight, and transferred onto fibronectin-coated Bioptechs DeltaT dishes. Once adhered, the 
cells were starved overnight, stimulated with $100 \mathrm{ng} / \mathrm{ml} \mathrm{EGF}$, and imaged using the Nikon TE2000S inverted microscope with attached Photometric Cool snap HQ CCD camera. Images were collected every 5 seconds for 20 minutes using the TRITC filter to visualize actin. The movies taken were saved as TIFF image sequences for import into ImageJ. Kymographic analysis was performed as described previously (160). Briefly, the multiple kymograph plugins were used to draw plots of protrusion dynamics. Persistence was the time from the initiation of protrusion until the initiation of retraction.

\subsection{9) Substrate-trapping, affinity precipitation and far western analysis}

Substrate trapping with the double-mutant (D425A and C459S) SHP2 (DM-SHP2) and affinity precipitation with the GST fusion of the PTP domain of DM-SHP2 (DM-PTP) were performed as described previously $(25,49)$. For Far Western analysis, FLAG-tagged FAK constructs (wild type, Y397F, and Y407F) were immunoprecipitated after plating cells on fibronectin to induce phosphorylation, and this enriched FAK was separated on SDS-PAGE before transfer onto nitrocellulose membranes. The membranes were then incubated overnight with $100 \mathrm{ng} / \mathrm{ml}$ of DM-PTP in TBST plus 5\% BSA overnight at $4{ }^{\circ} \mathrm{C}$, probed with anti-GST antibody for 2 hours at room temperature, and detected as described in the immunostaining section.

\subsubsection{0) In vitro phosphatase assay}

FAK-null MEFs expressing WT FLAG-FAK were seeded on fibronectin to activate FAK. The cells were lysed using iodoacetic acid to permanently inhibit endogenous phosphatases. 10 mM DTT was then added to quench the remaining iodoacetic acid. Equal amounts of protein (100 $\mu \mathrm{g}$ as assessed by the BCA assay) was added to a phosphatase reaction buffer at $\mathrm{pH} 7.2$ (25 mM HEPES, $150 \mathrm{mM} \mathrm{NaCl}$, and $1 \mathrm{mM}$ EDTA) along with $1 \mu \mathrm{g}$ of purified, recombinant SHP2 
lacking the $\mathrm{N}$-terminal SH2 domain. The reaction was allowed to proceed for the indicated time periods at $37^{\circ} \mathrm{C}$ before terminating with Laemmli sample buffer and boiling. The assay was run in parallel with no added PTP to control for possible residual endogenous PTP activity.

\subsubsection{1) Statistical analyses}

GraphPad Prism was used to make the graphs as well as to determine confidence intervals and run statistical tests. To gauge significance of data, unpaired, two-tailed t-tests were run with 95\% confidence intervals.

\section{4) Results}

\subsection{1) SHP2 is required for basal as well as EGF-stimulated BTBC cell motility}

Past work has shown SHP2 to be a critical factor in fibroblast migration through transwell assays (153). In addition, the importance of SHP2 in breast cancer cell migration has been suggested based on wound healing assays (161). Here, we wished to explore the impact of SHP2 inhibition on the rate of cell motility. To this end, we inhibited SHP2 protein levels in two prototypical BTBC cell lines, the MDA-MB231 and the MDA-MB468, using two shRNA that were previously demonstrated to be specific for silencing SHP2 expression (157). As shown in Fig.1A, both shRNA constructs silenced SHP2 protein expression by greater than $90 \%$. We then subjected these cells to a wound-healing assay under normal growing conditions. Inhibition of SHP2 with both shRNA-1 (data not shown) and shRNA-2 (Fig.1B) resulted in a clear loss of wound healing ability. Since we noted similar defects in wound healing using both shRNA-1 and 2 , we opted to continue further experiments with shRNA-2 due to its slightly better inhibition of protein expression, especially in MDA-MB468 cells. 
To obtain further insights on the impact of SHP2 inhibition, the rate of wound closure was estimated by calculating changes in wound dimension over 8 hours following scratching. The 8 hour time point was chosen because it represents a time when cells were still linearly migrating before contacting each other from opposite directions, especially in the controls. Multiple pictures of the monolayer wound were taken at the 0 and the 8 hour time points, and the cell-free area of each photograph was measured in microns using the Olympus Microsuite Basic Edition software. The average area was divided by 2190 microns, the image length of each photograph taken at $4 \mathrm{x}$ objective, to arrive at average wound width. Changes in wound width between the 0 and the 8 hour time points were used for comparison of the control and the shRNA cells. While the control MDA-MB231 and MDA-MB468 cells had reduced the wound width by an average of 325 and 461 microns in 8 hours, respectively, the corresponding shRNA cells had reduced by approximately 220 and 306 microns, respectively. In other words, while the control cells of MDA-MB231 and MDA-MB468 cells were closing the wound at a rate of 40 and 57 microns per hour, respectively, the corresponding shRNA cells were closing at a rate of 27 and 38 microns per hour, respectively (Table 1 and 2). Therefore, depletion of SHP2 retards wound healing.

SHP2 is well-known for its positive role in growth factor-related signaling, especially that of epidermal growth factor (EGF) (49). EGF is known to stimulate cell motility in both the MDA-MB-231 (162) and the MDA-MB-468 (163) cells, and EGF receptor is known to be overexpressed in a large number of triple-negative breast cancers. Therefore, we wished to determine whether SHP2 is required for EGF-stimulated cell migration. We performed similar measurements to estimate wound closure rate over 2 hours in the presence of EGF (100 $\mathrm{ng} / \mathrm{mL}$ ). This time point was chosen based on our previous studies where we demonstrated 
that EGF-induced signaling drops significantly within 2 hours due to degradation of the receptor (164). EGF stimulation induced a modest motility response in the controls, resulting in a $20-30 \%$ increase in wound healing rate, but this response was not observed in the shRNA cells (Fig.1D and E). These results suggest that SHP2 can exert control over EGF-induced cell movement, which is unsurprising given its known role as a promoter of growth factor receptor signaling.

\subsection{2) SHP2 promotes proper cell directionality during wound healing}

Previous studies have shown that inhibition of SHP2 induces marked changes in cell morphology (165), indicating that SHP2 may play a role in cell polarization. To test this hypothesis, we subjected BTBC cells to wound-healing on fibronectin-coated glass coverslips and stained the golgi body, since this organelle becomes oriented toward the wound in response to cell polarization (159). In both the MDA-MB231 and the MDA-MB468 cell lines, inhibition of SHP2 expression resulted in a significant loss of the population of cells with golgi oriented toward the wound (Fig.2A and B). Control cells tended to orient toward the wound approximately $60 \%$ of the time, while SHP2-depleted cells oriented about $40 \%$ of the time (Fig.2C), indicating that SHP2 is indeed critical for the establishment of cell polarity during migration in BTBC cells.

\subsection{3) SHP2 is recruited to the leading edge of BTBC cells}

The impaired polarization and migration of SHP2-depleted cells suggested that SHP2 may function at the cell membrane to promote motility, which is not surprising sinceSHP2 is primarily known to act at the plasma membrane (166). Using immunofluorescence, we analyzed for SHP2 localization under steady-state conditions (Fig.3).We co-stained 
endogenous SHP2 along with cortactin, a marker of the leading edge, in the MDA-MB231

(Fig.3A) and the MDA-MB468 (Fig.3B) cells.SHP2 was found prominently colocalized with cortactin at the leading edges of the control cells, and, as expected, this signal was not readily detectable in SHP2-depleted cells, in the meantime serving as a specificity control for the antiSHP2 antibody. These results strongly indicate that SHP2 is recruited to the leading edge of cells.

\subsection{4) SHP2 depletion alters EGF-stimulated membrane protrusions in BTBC cells}

The presence of SHP2 at the cell periphery along with the effects of SHP2 depletion on EGFstimulated motility led us to hypothesize that SHP2activity may exert control over membrane protrusions, specifically the dynamics of lamellipodia. We transfected the MDA-MB231 and the MDA-MB468 cells with mCherry-actin (Clontech) to visualize actin-rich lamellipodia in realtime. These cells were seeded on fibronectin-coated DeltaT (Bioptechs) dishes prior to stimulation with EGF, and kymographs of individual membrane protrusions were generated using ImageJ (Fig.4A). In general, SHP2 depletion resulted in protrusions showing more sharp peaks, suggesting a defect in persistence. Lamellipodia persistence was further quantified as described by Bryce, et al (167) to better characterize the result of SHP2 depletion. Persistence was measured as the time from initiation of the protrusion to initiation of retraction. While persistence in the control MDA-MB231 cells was about 60 seconds, it was only about 30 seconds in the corresponding shRNA cells, an approximately 50\% drop caused by SHP2 inhibition (Fig.4B). The drop in lamellipodia persistence in the MDA-MB468 cells caused by SHP2 silencing was even more drastic. While the control cells showed an approximately 220 seconds persistence, the corresponding shRNA cells exhibited an average of 70 seconds, an approximately 70\% drop in persistence (Fig.4C). Importantly, in both MDA-MB231 and MDA- 
MB468 cells, depletion of SHP2 resulted in lamellipodial persistence induced by EGF that was indistinguishable from non-stimulated cells, mirroring the defects in EGF response shown in

Fig. 1D and 1E. These data suggest that SHP2 is especially important for control of protrusion persistence.

\subsection{5) pY397 in FAK is a target for SHP2 PTPase activity}

To obtain insights on the mechanistic of SHP2 regulation of cell migration, substrate-trapping studies were conducted in mouse embryonic fibroblasts (MEFs) in which SHP2 was previously shown to regulate the activity of focal adhesion proteins (165).FLAG-tagged wild type SHP2 (WT-SHP2), substrate-trapping SHP2, also referred to as DM-SHP2 (DM for D425A and C459S double mutant that cannot catalyze hydrolysis but retains substrate binding capability) or vector alone was expressed in MEFs as described in the materials and methods and in previous reports (25). Cells were seeded on fibronectin-coated plates to stimulate tyrosine phosphorylation of adhesion proteins, and lysates prepared from them were subjected to immunoprecipitation with anti-FLAG antibody. Immunostaining with anti-pTyr antibody showed coprecipitation of an approximately $120 \mathrm{kDa}$ protein with the DM-SHP2, but not with vector or the WT-SHP2 (Fig.5A, top), suggesting that the co-precipitation was due to substrate trapping as reported previously. Based on previous suggestion that FAK might be an SHP2 substrate $(64,67)$ the same preparations were probed with anti-FAK antibody. Indeed, the 120 kDa tyrosine-phosphorylated protein trapped by the DM-SHP2 was FAK (Fig.5A, middle). Reprobing with anti-FLAG antibody showed that both WT-SHP2 and DM-SHP2 proteins were effectively precipitated although the WT-SHP2 protein level was higher than the DM-SHP2 (Fig.5A, bottom). These results confirm previous studies which suggested that FAK is an SHP2 substrate. 
Previously identified SHP2 substrates possess acidic amino acids N-terminal to the target phosphotyrosine $(25,49)$. We thus suspected that Tyr397 andTyr407 (Y397 and Y407), which have such residues in the immediate $\mathrm{N}$-terminal region, might be substrates. To test this hypothesis, FLAG-tagged wild type or mutant FAK (Y397F and Y407F) were expressed in FAKnull $\left(\mathrm{FAK}^{\circ} / \mathrm{)}\right) \mathrm{MEFs}$, and lysates prepared from these cells were subjected to affinity precipitation with GST-DM-PTP. As shown in Fig.5B, precipitation of FAK required pY397, since its mutation abrogated binding. On the other hand, mutation of Y407 did not affect binding, suggesting that it was not a target for SHP2. Reprobing with anti-GST antibody confirmed that the amount of GST-DM-PTP was comparable in all lanes. Analysis of input total cell lysates showed that the three FAK proteins were expressed comparably (Fig.5C). In order to eliminate the possibility of indirect interaction, we performed Far Western analysis (Fig.5D). In this assay, FLAG-tagged FAK proteins described above were immunoprecipitated from MEFs seeded on fibronectin, resolved by polyacrylamide gel electrophoresis, transferred onto a nitrocellulose membrane, blocked with BSA and probed with the GST-DM-PTP as described in the materials and methods. Anti-GST was then used to detect SHP2 binding. The results closely mimicked the affinity precipitation showing that pY397 was required for the binging of DM-PTP. Reprobing with anti-FLAG antibody showed that the amount of the different FAK proteins recovered from the immunoprecipitation reactions was comparable. These binding results suggest that SHP2 directly regulates FAK by acting on pY397.

In order to assess the ability of wild type SHP2 to directly dephosphorylate FAK in vitro, we subjected MEF cell lysates expressing FLAG-tagged FAK to incubation with purified GSTWT-PTP lacking the regulatory SH2 domains. Immunostaining with anti-pY397 and anti-pY576 showed that SHP2 preferentially dephosphorylated pY397, but not pY576 (Fig.5E). Reprobing 
with anti-FLAG antibody showed the presence of comparable amount of FAK protein in all lanes. These results clearly show that pY397 in FAK is a substrate for SHP2 which in turn suggest that SHP2 regulates FAK activity by acting directly on the autophosphorylation site, pY397.Our results partially disagree with a previous report that suggested that both pY397 and pY576 are SHP2 substrates (168). The observed discrepancies might have been caused by differences in experimental approaches. In the current work, purified PTP was used as an enzyme as opposed to immunoprecipitated SHP2, a step that cannot exclude coprecipitation of other PTPs. The use of purified PTP also allows accurate control of enzyme concentration. Furthermore, we did employ iodoacetate treatment of total cell lysates containing FAK to irreversibly inhibit endogenous PTPs before PTPase reaction, a step not taken by the other report (168).

We next wished to confirm in the BTBC cell lines whether FAK pY397 could be mediated by SHP2. To this end, we seeded cells on fibronectin coverslips and gave them sufficient time to attach and spread. Immunofluorescence against pY397 showed a differential localization when SHP2 was inhibited. Focal adhesions containing pY397 appeared larger and more numerous than in controls, suggesting a role for SHP2 in the regulation of these complexes and the level of pY397 during adhesion and spreading (Figs 5F and 5G).

\subsection{6) SHP2 promotes FAK inactivation to promote EGF-stimulated wound healing}

The results described in Fig.1D and E, demonstrated that SHP2 was required for EGF-induced wound healing, while those presented in Fig. 5 showed that SHP2 modulates FAK tyrosine phosphorylation by acting on pY397, the autophosphorylation site known to regulate FAK activity. It was thus reasoned that SHP2 mediates EGF-stimulated cell migration by 
inactivating FAK and the phenotype induced by silencing SHP2 is a result of FAK hyperphosphorylation. We first examined the role of SHP2 in the regulation of pY397 levels during EGF stimulation. Confluent monolayers of MDA-MB231 (Fig. 6A) and MDA-MB468 (Fig. 6B) cells were serum-starved overnight before stimulation with EGF. In the MDA-MB231 control cells, EGF induced no significant change in the pY397 signal, while the MDA-MB468 cells exhibited a modest increase of approximately $20 \%$. In both cell lines, depletion of SHP2 caused no significant change to pY397 under serum-starved conditions, but stimulation by EGF in both cases induced a marked activation of FAK. In MDA-MB231 shRNA cells, EGF stimulated resulted in an approximately 50\% increase of pY397, whereas in the MDA-MB468 cells the increase was nearly $80 \%$ (Fig. 6A and 6B, lower panels). These results clearly indicate SHP2 is essential for preventing FAK hyperactivation under EGF stimulation.

SHP2 appeared to be playing an antagonistic role in FAK activation during EGF stimulation. Therefore, PF-573228, a FAK inhibitor (169), was used to inhibit kinase activity. This compound is known to completely inhibit focal adhesion kinase at $10 \mu \mathrm{M}$ concentration and above, but we hypothesized that if the role of SHP2 was to fine-tune the dynamic regulation of FAK phosphorylation, then adding a lesser amount of the inhibitor should rescue some of the defects we observed in SHP2-depleted cells. To this end, we applied 10n M PF573228 to BTBC cells in the EGF-induced assays.

First, we noted that treatment with PF-573228 had no effect on the pY397 levels in control MDA-MB231 cells. In MDA-MB468 cells, we noted a slight decrease equating to approximately 20\%. Despite the low to nonexistent inhibition of pY397 at these concentrations, we found that FAK inhibition was able to completely prevent the 
hyperactivation induced by EGF treatment in the shRNA cells. Thus, we assessed EGFstimulated wound healing experiments in a manner similar to Figs. 1D and 1E. We observed that a concentration of $10 \mathrm{nM} \mathrm{FAK}$ inhibitor had no significant effect on the rate of wound healing in control and shRNA BTBC cell lines under basal growing conditions; however, when the FAK inhibitor was applied to shRNA cells followed by EGF stimulation, we noted an increase in wound healing rate of approximately $20-30 \%$ in the shRNA cells (Fig.7A and B), a difference similar to the EGF-induced increase seen in control cells (compare with Fig.1D and E).

Since aberrant FAK activation has been linked to decreased nascent adhesion strength $(64,67)$, we reasoned that the FAK inhibitor could rescue the defects in EGF-stimulated lamellipodia persistence observed upon SHP2 depletion. The 10 nM PF-573228 FAK inhibitor was able to reverse the loss of persistence as evidenced by the occurrence of long-lasting protrusions (Fig.7C and D) similar to those seen in the control cells (Fig.4). We also wished to determine whether FAK inhibition could rescue the defects observed in cell polarity. We repeated the experiments presented in Fig. 2 in the presence and absence of $10 \mathrm{nM} \mathrm{PF-573228}$ (Fig. 7E). The inhibitor showed no alterations in the percentage of cells that were properly oriented during wound healing. Taken together, these results indicated that SHP2 was involved in attenuating FAK phosphorylation during EGF treatment. This fine-tuning of FAK activation resulted in increased wound healing after treatment with EGF, presumably through the promotion of lamellipodia persistence. 


\section{5) Discussion}

Cell migration is one of the mechanisms utilized by a tumor cell to locally invade and eventually metastasize to distant tissues and organs to initiate secondary tumors, which is often the cause of death from cancer. SHP2 has been known to influence cell migration for more than a decade $(63,153,165)$, and much of the study into this phenomenon has focused on its putative regulation of either focal adhesion dynamics $(64,67)$ or RhoA activity $(155,170,171)$. In this report, we have shown for the first time that SHP2 regulates lamellipodia dynamics and golgi orientation during motility.

In order to assess the significance of SHP2 in BTBC, the highly metastatic form of breast cancer, we investigated whether or not it plays a role in cell migration. In line with this goal, we have demonstrated that SHP2 is required for BTBC cell migration (Fig. 1). These findings agree with our own and other previous reports where a positive role for SHP2 in cell migration was demonstrated $(25,67,150)$, although those reports utilized different cell contexts. Furthermore, the current data provide additional insight by showing that SHP2 promotes cell migration by enhancing cell polarization, the pathfinding step in cell migration.

SHP2 is a well-known effector of EGFR signaling, a receptor tyrosine kinase overexpressed in approximately $70 \%$ of BTBC $(172,173)$, including the cell lines used in this study, the MDA-MB231 and the MDA-MB468. It was thus logical to test if SHP2 is required for EGF-induced cell migration under these contexts. We have found that both cells were unresponsive to EGF-induced wound healing when SHP2 was depleted (Fig. 1D), suggesting that SHP2 plays essential roles in EGFR-induced migration of BTBC cells in addition to its regulation of basal cell migration (Fig.1B). This regulation of EGF-dependent migration by 
SHP2 is not trivial since EGFR is regarded as a contributing factor for the aggressive manifestations of BTBC $(174,175)$. Our findings imply that SHP2 may prove to be an effective therapeutic target in triple-negative breast cancers.

Data presented in Fig.2 demonstrate that cells in which SHP2 is depleted show defects in their ability to orient themselves toward the space created by the monolayer wounding. These results clearly demonstrate that one of the mechanisms by which SHP2 promotes cells migration is by mediating proper cell polarization. Recruitment of SHP2 to the leading edge of a migrating cell (Fig. 3) supports this possibility and suggests a direct role for SHP2 in cellular path finding. This is not surprising given the evidence that SHP2's cellular function is enhanced by its interaction with tyrosine-phosphorylated proteins at the plasma membrane (176).

SHP2 appears to be important in EGF-induced lamellipodia persistence (Fig. 4), an event that allows effective lamellipodia extension and forward cellular movement. Lamellipodia persistence requires temporal strengthening of nascent adhesions or maturation of nascent adhesion complexes to focal adhesions. The recruitment of SHP2 to the leading edge of a migrating cell (Fig.3) suggests its regulation of lamellipodia persistence by acting on adhesion proteins like paxillin and focal adhesion kinase (FAK) which were previously suggested to be SHP2 substrates $(168,177)$. In support of this possibility, a previous work in SHP2-null fibroblasts showed instability of focal adhesion proteins at ECM-cell contacts which could be rescued either with dominant negative FAK or with reintroduction of wildtype SHP2 (67). Under these conditions, FAK has been shown to be hyper-activated, suggesting that it counteracts maturation of nascent complexes to focal adhesions. Indeed, hyper-activated FAK has been shown to sequester paxillin away from nascent adhesion, leading to weakening of 
these adhesions and presumably inhibiting lamellipodia persistence (168). These findings lend support to the possibility that SHP2 regulation of FAK tyrosine phosphorylation plays a key role in lamellipodia persistence.

By employing intracellular substrate-trapping studies and in vitro phosphatase assays, we have demonstrated that FAK is an SHP2 substrate (Fig.5), which is in agreement with previous suggestions (168). We have further shown that the preferred site of SHP2 action in FAK is pY397 as opposed to the closely-related pY576 and pY407. This preference for pY397 suggests that SHP2 not only regulates FAK autophosphorylation, but also subsequent recruitment of Src, preventing further activation. Therefore, our findings are consistent with SHP2 promoting cell migration by enhancing lamellipodia persistence through down regulation of FAK activity. Cell migration is a dynamic process that involves adhesion and deadhesion to the ECM. At this stage, it is not known whether or not SHP2 is also involved in the deadhesion process, and if yes, what substrates it acts on to promote deadhesion. It will be interesting to address these questions in future studies.

The observation that mild FAK inhibition is able to rescue the EGF responsiveness in SHP2-depleted BTBC cells (Figs. 6 and 7) provides further support to the idea that SHP2 acts to down regulate FAK to promote nascent adhesion strengthening. Strengthening of focal adhesions by antagonizing FAK signaling was also noted in the initial characterization of the inhibitor (169). In that study, concentrations of the drug that inhibited the majority of FAK in cells were shown to strongly inhibit cell motility in part due to the lost focal adhesion dynamics. Taken together, excessive FAK activation will result in increased focal adhesion dynamics and low traction, leading to poor motility, while a low level of FAK activity promotes 
the anchorage of cells through the aberrant formation of non-dissociable focal adhesions. Therefore, fine tuning FAK activity seems to be essential for EGF-induced lamellipodia persistence and strengthening of focal complexes to focal adhesions. Our data suggest that SHP2 attenuates FAK activity to promote EGF-induced cell migration. Interestingly, FAK inhibition did not rescue the wound healing defects under normal growing conditions observed upon SHP2 inhibition, suggesting the existence of other FAK-independent mechanisms for control of cell migration by SHP2. This observation warrants further investigation in future studies.

\section{6) Acknowledgements}

We thank NIH-NCI and DOD Breast cancer Research Program for Financial support. Imaging experiments and image analysis were performed in the West Virginia University Microscope Imaging Facility, which has been supported by the Mary Babb Randolph Cancer Center and NIH grants P20 RR016440, P30 RR032138/GM103488 and P20 RR016477.

This work was supported by grant number CA124940 from the National Cancer Institute (NCI), a component of the National Institute of Health (NIH) to YMA.ZRH is supported by the Department of Defense Breast Cancer Research Program fellowship award number W81XWH-11-1-0133.

\section{7) Figure Legends}

Figure 1.

A, silencing SHP2 protein expression in the MDA-MB231 and the MDA-MB468 cells using 2 specific shRNA constructs designated as shRNA-1 and shRNA-2. Anti- $\beta$-actin immunostaining was used as a loading control. Control and shRNA cells derived from the MDA-MB231 (B) and 
the MDA-MB468 (C) were grown to confluence followed by wounding with a P200 pipette tip. Images of wounds were taken at 0- and 24-hour time points. Rate of wound closure per hour in 8 hours in the MDA-MB231 (D) and the MDA-MB468 (E) was estimated by dividing the overall change in wound space similar to that described in Table 1 . Data presented are mean \pm SEM. ***, $P<0.001$ and ${ }^{* *}, P<0.01$. Representative images of control and shRNA cells derived from the MDA-MB231 (F) and MDA-MB468 (G) stained with 4',6-diamidino-2-phenylindole (DAPI) and GM130 after 6 hours of wound healing are shown. Arrowheads represent cells that were considered as oriented to the wound. $\mathrm{H}$, Golgi orientation was calculated as a percentage of cells at the wound edge with stained Golgi facing the wound space. Ten fields of cells were gathered per independent experiment, totaling more than 500 cells for each cell line. Data are presented as mean \pm SEM.

\section{Figure 2.}

SHP2 localizes to the leading edge of BTBC cells. The control and the shRNA cells derived from the MDA-MB231 (A) and the MDA-MB468 (B) lines were seeded on fibronectin-coated coverslips and were allowed to attach overnight. Cells were then fixed, permeablized, and stained with anti-cortactin and anti-SHP2 antibodies. Exposure times to acquire images of SHP2 staining were held constant between control and shRNA slides. Arrows indicate leading edges of cells.

\section{Figure 3.}

Bar graphs showing the impact of SHP2 silencing on EGF-induced wound healing over a period of 2 hours in the MDA-MB231 (A) and the MDA-MB468 (B) cells. SHP2 depletion alters EGFstimulated membrane protrusions in BTBC cells. C, kymographs of movies collected from livecell imaging experiments for both the MDA-MB231 and MDA-MB468 cells are shown. 
Kymographs were used to calculate the persistence of the lamellipodia in the MDA-MB231 (D) and the MDA-MB468 (E) cells in a manner described by Kelley and colleagues (160). Data presented are mean \pm SEM of protrusion persistence time in seconds collected from at least 10 cells per cell line. ns: nonsignificant. ${ }^{*}, P<0.05$.

\section{Figure 4.}

SHP2 regulates FAK activity in BTBC cells. A, substrate-trapping studies in MEFs expressing vector alone, FLAG-WT-SHP2, or FLAG-DM-SHP2. Cells were seeded on fibronectin for 30 minutes, and lysates prepared from these cells were subjected to anti-FLAG immunoprecipitation followed by immunostaining analysis with anti-pTyr, anti-FAK, and antiFLAG (for SHP2) antibodies. B, in vitro affinity precipitation with GST-DM-PTP of cell lysates prepared from FAK-null MEFs expressing vector alone or FLAG-tagged FAK proteins. The precipitates were analyzed by immunostaining with ant-FLAG antibody for FAK proteins and with anti-GST for GST-DM-PTP. C, input total cell lysates used for affinity precipitation were stained with anti-FLAG, anti-pY397, and anti- $\beta$-actin antibodies. D, far Western blot analysis with GST-DM-PTP to show that the presence of pY397 is needed for binding. E, in vitro phosphatase assay using WT FAK as a substrate and purified WT SHP2 as an enzyme. Immunostaining results for pY397, pY576, and FAK (FLAG-FAK) are shown. NP, no PTP.

\section{Figure 5.}

Analysis of impact of SHP2 silencing on the pY397-FAK levels. The control and the shRNA cells derived from the MDA-MB231 (A) and MDA-MB468 (B) were grown to about $90 \%$ confluence, serum-starved for approximately 4 hours, and then left unstimulated or stimulated with 100 ng/mL EGF for 20 minutes. Lysates prepared from these cells were analyzed by 
immunostaining with anti-pY397 antibody. Bar graphs show band density measurements of the anti-pY397 staining from 3 independent experiments. The MDA-MB231 (C) and the MDAMB468 (D) cells grown on coverslips were stimulated with the same concentration of EGF and then analyzed by immunofluorescence microscopy after staining with anti-pY397 antibody. The 4',6-diamidino-2-phenylindole (DAPI) staining also was conducted to show the nucleus.

\section{Figure 6.}

Effect of FAK inhibition on EGF-induced wound healing, lamellipodia persistence, and Golgi orientation. SHP2-depleted MDA-MB231 (A) and MDA-MB468 (B) cells were grown to confluence before scratching with a pipette tip. Cells were then fed with serum-free medium and left untreated or treated with $10 \mathrm{ng} / \mathrm{mL}$ FAK inhibitor (PF) and $100 \mathrm{ng} / \mathrm{mL}$ EGF. Rate of wound healing was assessed in the same manner as in Fig. 3A and B. Live-cell imaging was used to assess EGF-induced lamellipodia persistence in the presence and absence of PF (10 nmol/L) in theMDA-MB231 (C) and the MDA-MB468 (D) cells. Data presented are mean \pm SEM of protrusion persistence time in seconds collected from at least 10 cells per cell line. E, effect of the FAK inhibitor on Golgi orientation. Analysis of pY397-FAK levels in the MDA-MB231 (F) and MDA-MB468 (G) cells in the presence and absence of FAK inhibitor and EGF. ${ }^{*}, P<0.05$ 
Figure 1.

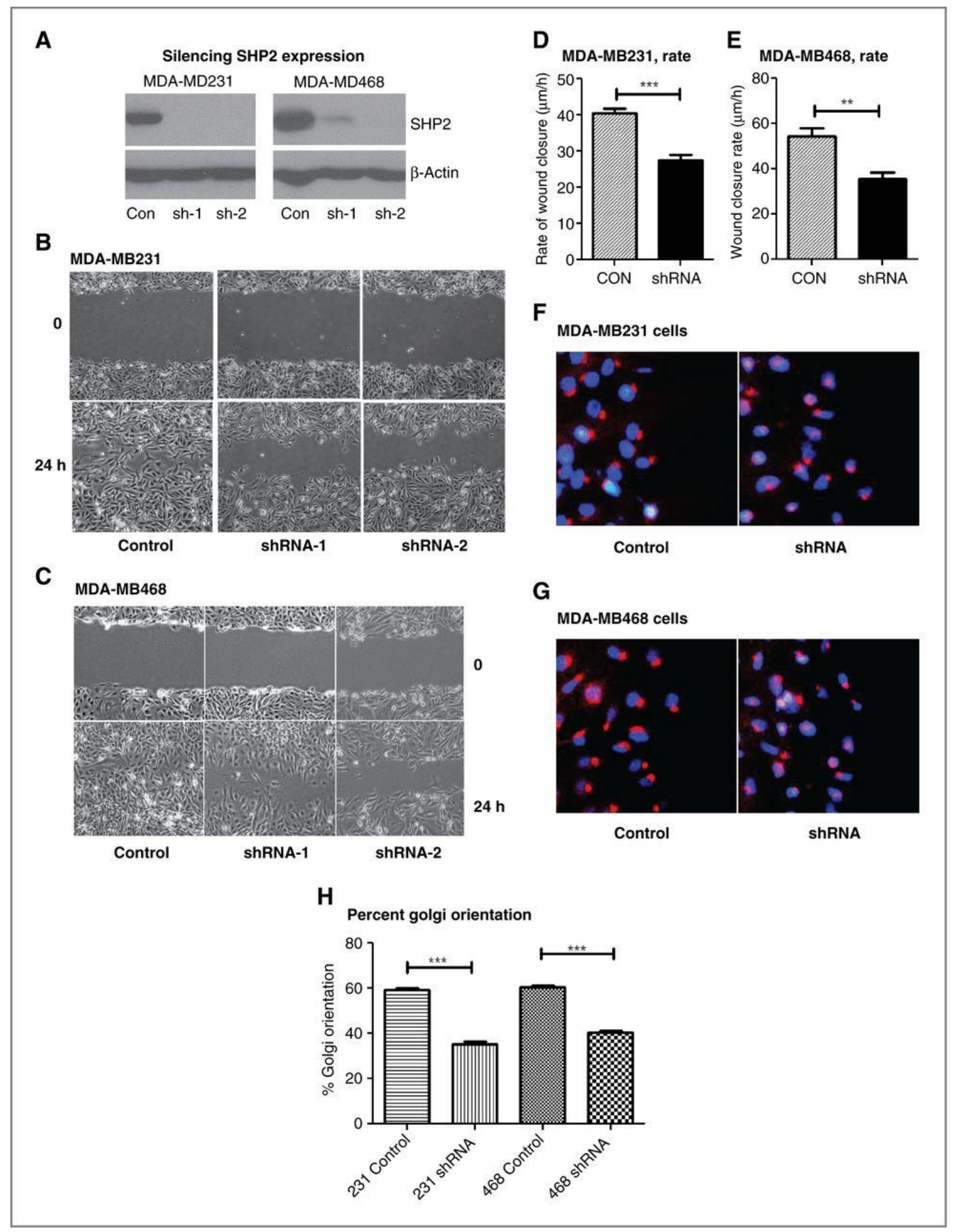


Figure 2.

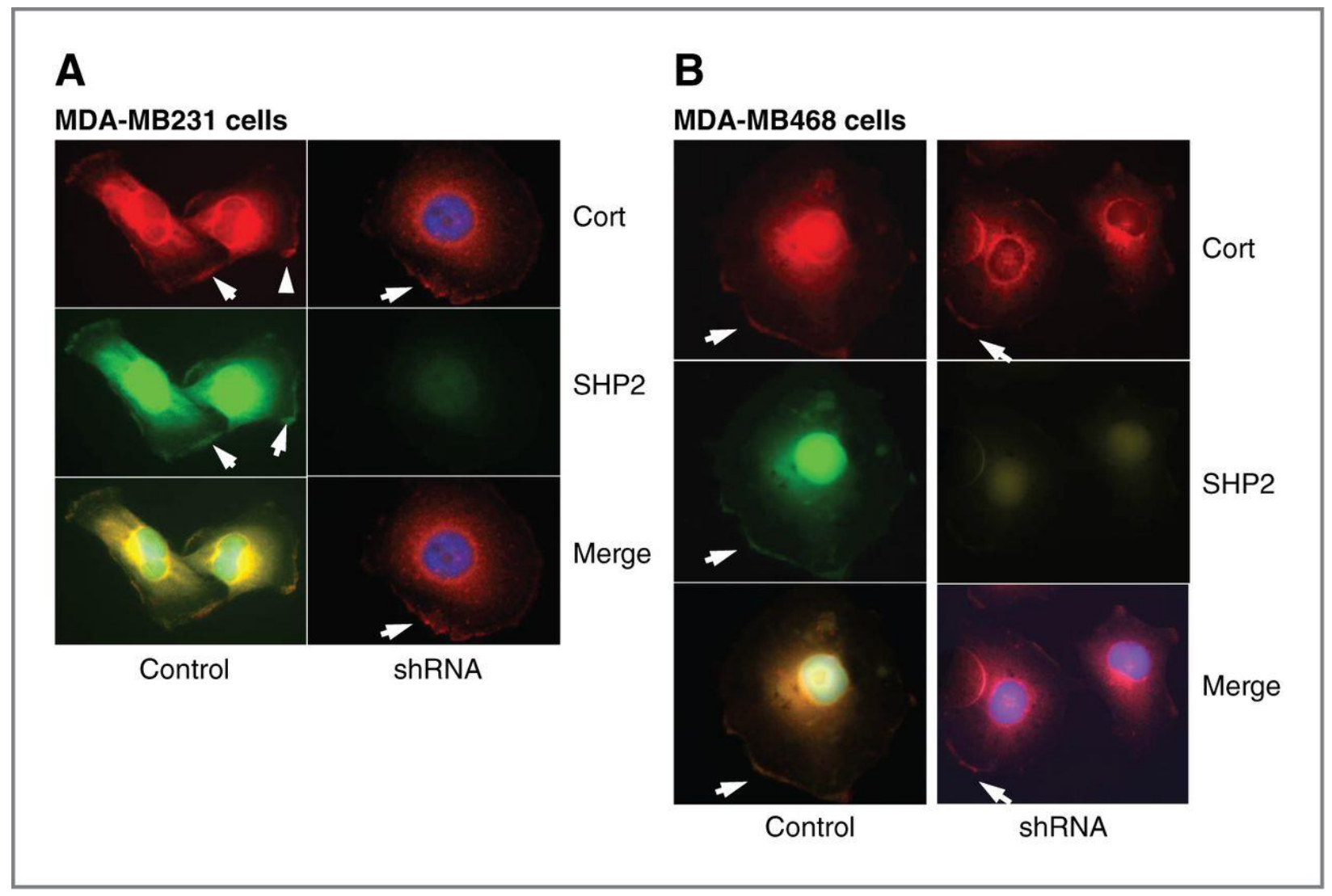


Figure 3.
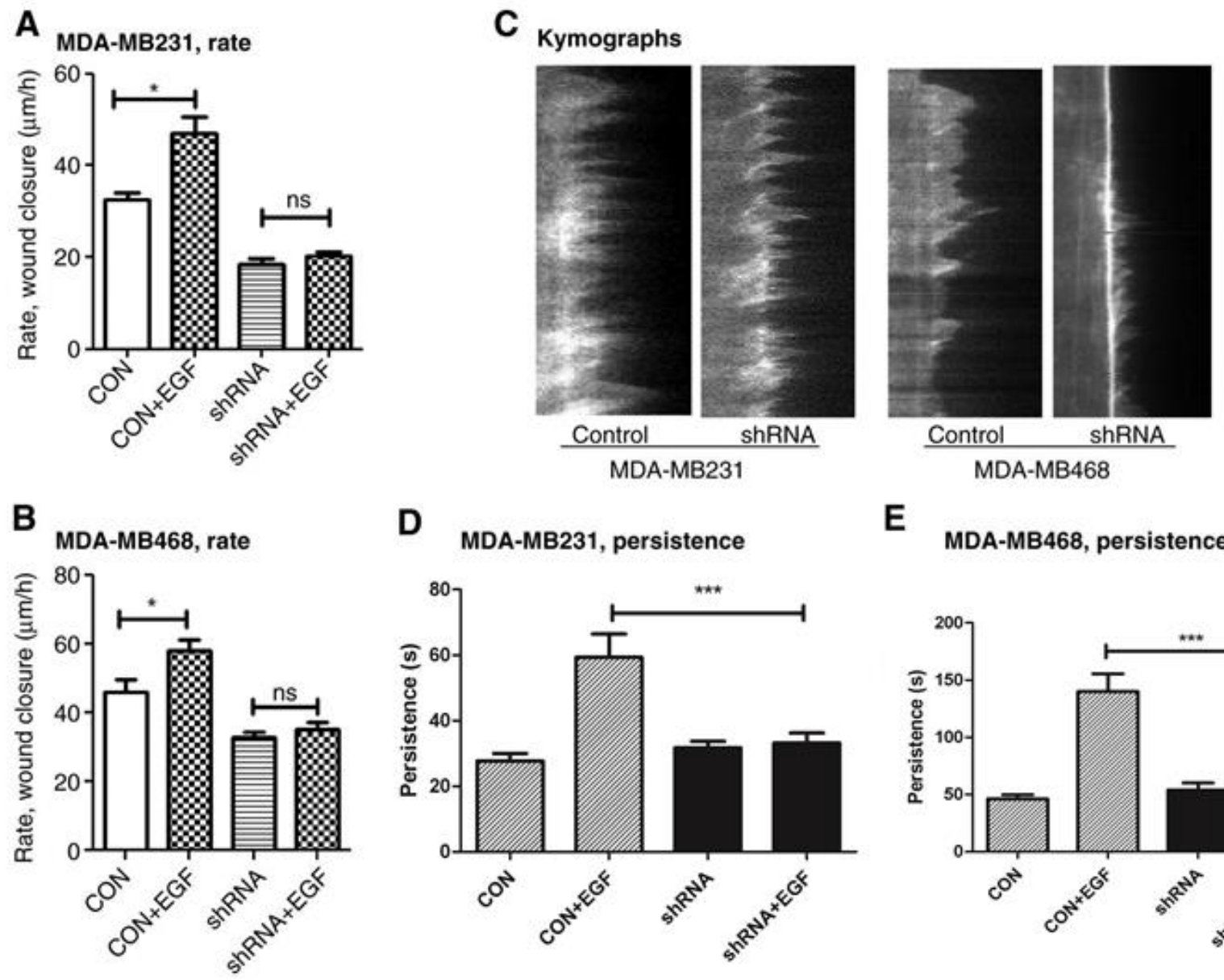

D MDA-MB231, persistence
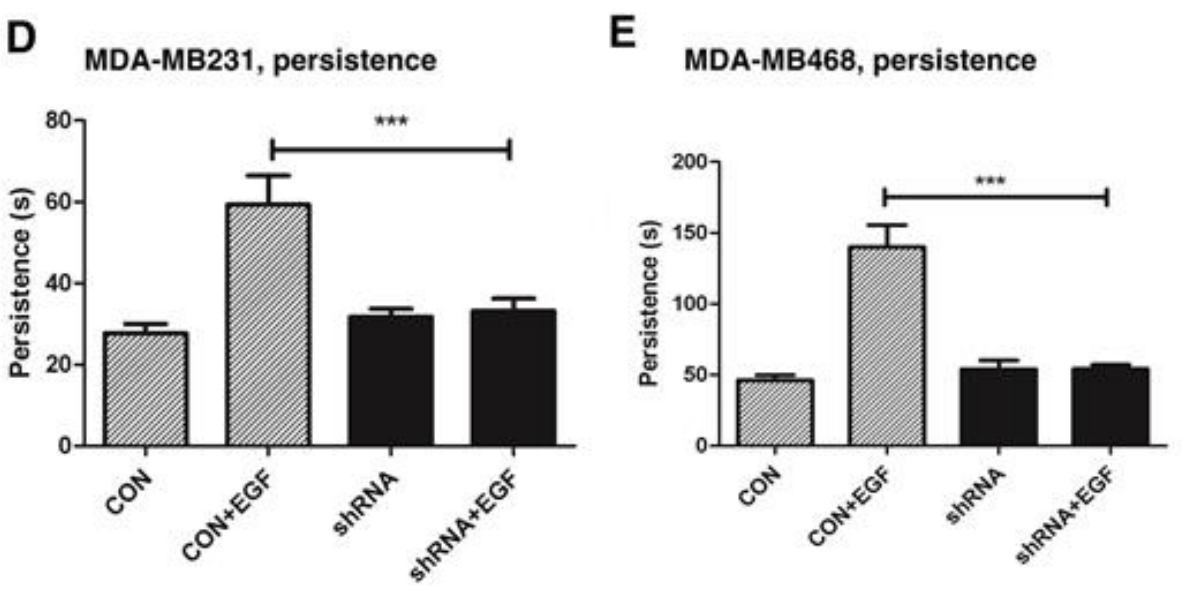
Figure 4.

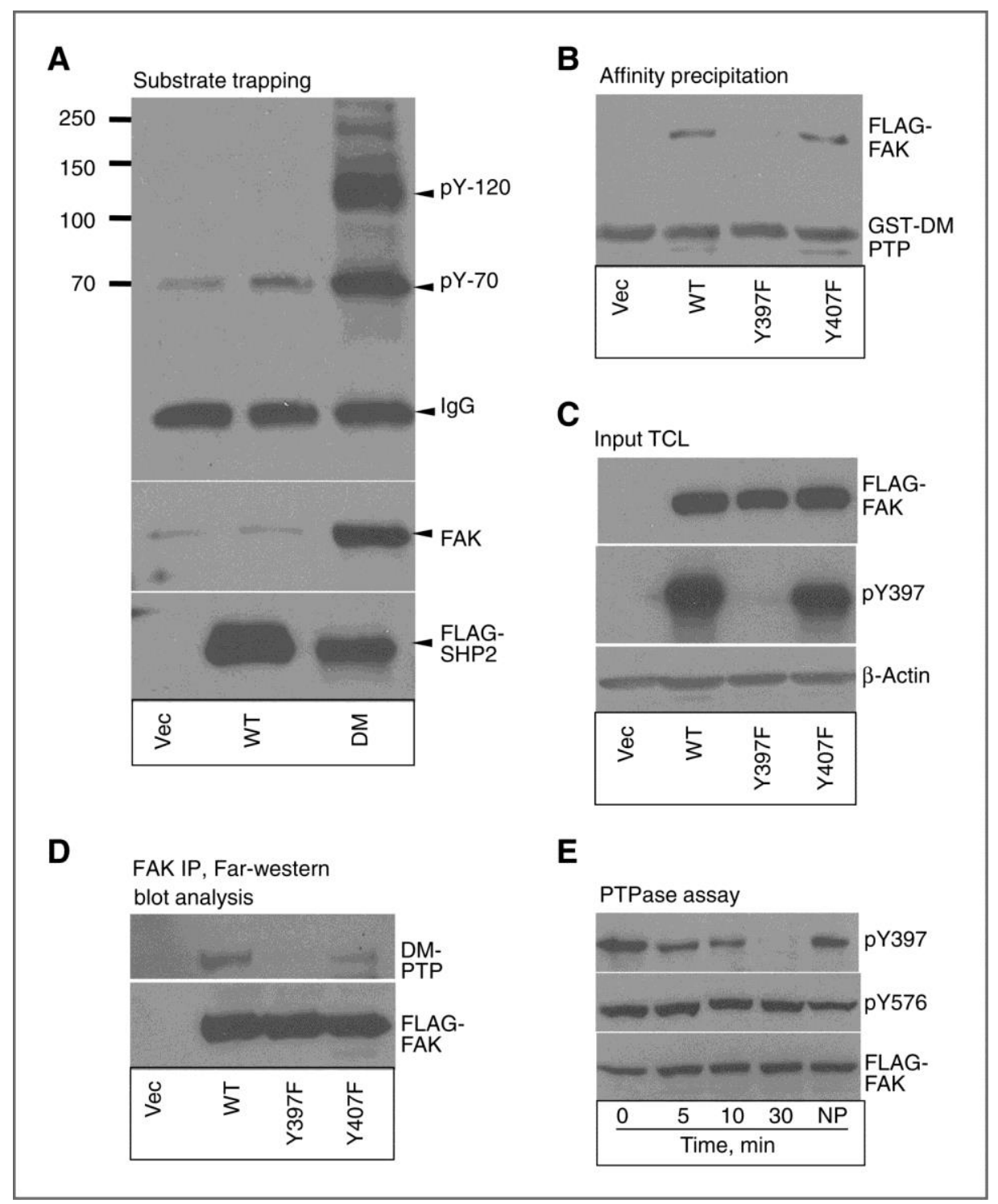


Figure 5.

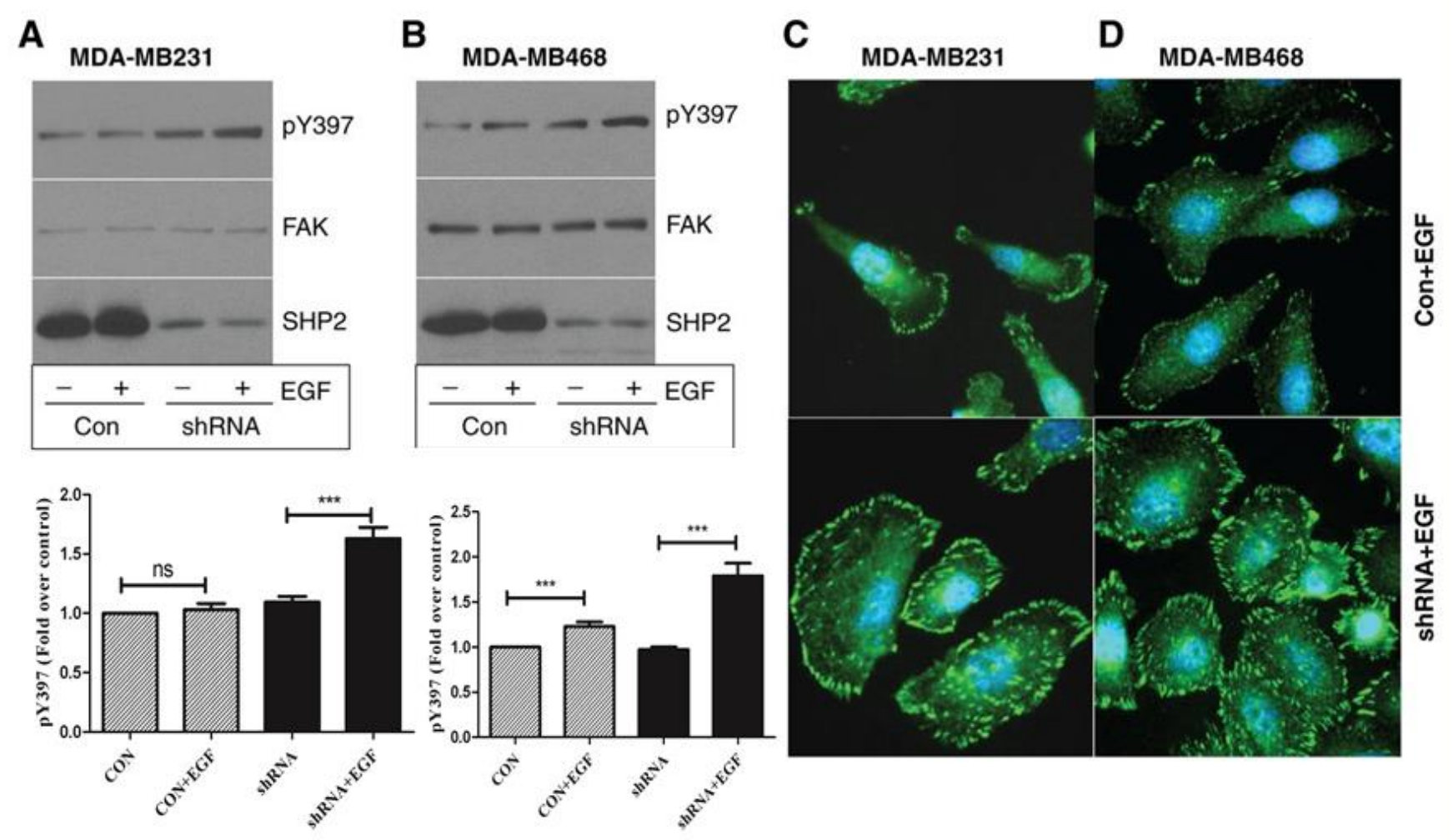


Figure 6.
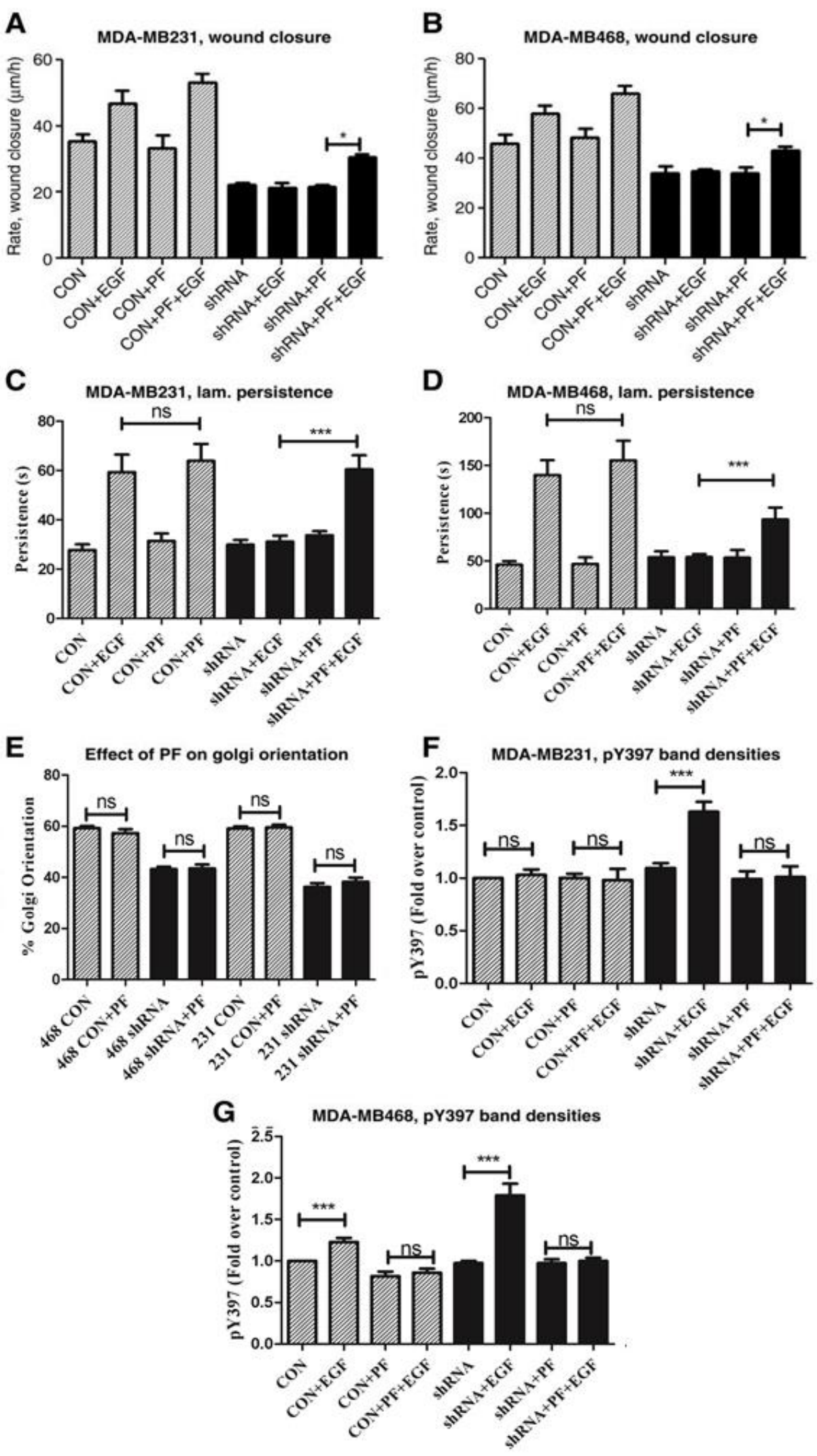


\section{5) Substrate discrimination by the Src homology 2-containing protein tyrosine phosphatase SHP2 predicted by molecular docking}

\section{1) Abstract}

The tyrosine phosphatase SHP2 is a positive regulator of mitogenic and cell survival signaling. In the signaling of receptor tyrosine kinases, especially EGFR and HER2, SHP2 is known to act on binding sites for RasGAP. However, it is not clear exactly how SHP2 recognizes these sequences selectively. In this report, a substrate-based peptide is shown to be a selective inhibitor for SHP2 over its homologue SHP1. The proto-oncogene HER2 was expressed in MCF-10A cells, and a mutation upstream of the phosphotyrosine 1023 abolished association with the SHP2 active site. This dissociation of the SHP2-HER2 interaction correlated with impaired EGF-induced signaling and transformation normally induced by HER2. Computational docking of the substrate-derived peptide identified SHP2 arginine 362 and lysine 364 as key mediators of binding. These data suggest key substrate-protein interactions that allow for selective binding.

\section{2) Introduction}

The Src homology 2-containing protein tyrosine phosphatase 2 (SHP2) is a cytosolic PTP that mediates many important signaling networks (178). It contains two tandemly-arranged SH2 domains that generate intramolecular autoinhibition of the catalytic active site. Binding to phosphotyrosine induces a conformational change that relieves this inhibition (39). When active, SHP2 functions to promote signaling related to proliferation $(179)$, survival $(180,181)$, and motility (153). This positive functionality of SHP2 is critical for promotion of different forms of cancer (52). In cells, SHP2 is required for maintenance of the transformed phenotype 
of fibroblasts expressing an oncogene (115). It is also required to promote the mesenchymal state and anchorage-independent survival in breast cancer cell lines (161). Thus, targeting SHP2 with small molecules appears to be an attractive means of combating cancer, but development of these inhibitors has proved challenging.

To date, numerous studies have been published characterizing inhibitors based on highthroughput chemical and in silico drug screens (182-186). A few common challenges have appeared through this work. First, moieties used to mimic phosphotyrosine tend to be charged, rendering the compound impermeable to the cell membrane. Selectivity of these compounds is another significant issue. The active sites of PTPs are well-conserved in sequence and overall charge distribution (187). Thus, many drugs that target SHP2 tend to target other PTPs, especially its homolog SHP1. This is an especially-important issue to overcome since SHP1 is a negative mediator of signaling (188). The most selective inhibitors for SHP2 have been shown to achieve approximately 20 -fold selectivity for SHP2 over SHP1 (184), which leaves significant room for improvement.

SHP2 is known to selectively bind substrates in vitro (189). This observation suggests that the issue of drug selectivity can be surmounted. Unfortunately, the intermolecular determinants of selective substrate binding to SHP2 have not been elucidated. A study exploring the peptide sequence best-recognized by SHP2 has been published (190). The specific molecular interactions required for selective binding remain to be elucidated, however. Numerous crystal structures of unbound (39) and small molecule inhibitor-bound SHP2 (186) have been published, but to date no study has been undertaken to elucidate the molecular determinants of a substrate binding to SHP2. To this end we have performed docking experiments along 
with biochemical validation to test how a substrate-based inhibitory peptide is able to selectively inhibit SHP2 over SHP1. The results presented herein suggest the presence of key

electrostatic and steric interactions that allow the peptide to bind SHP2 but not SHP1 and help pave the way toward selective SHP2 inhibitor design.

\section{3) Materials and Methods}

\subsection{1) Cells, cell culture, and reagents}

The immortalized mammary epithelial cell line MCF-10A were purchased from American Tissue Culture Collection (ATCC). These cells were cultured in Dulbecco's modified Eagle's medium supplemented with $10 \mu \mathrm{g} / \mathrm{ml}$ recombinant human insulin, $20 \mathrm{ng} / \mathrm{ml}$ EGF (PeproTech), $0.5 \mu \mathrm{g} / \mathrm{ml}$ hydrocortisone, $100 \mathrm{ng} / \mathrm{ml}$ cholera toxin (Sigma), and 5\% horse serum. Other reagents used included the DiFMUP kit (Invitrogen) and glutathione-sepharose beads (GE Healthcare); peptides corresponding to the SHP2 target site of EGFR (DADEpYL) were synthesized and purified to greater than or equal to $95 \%$ by NeoPeptide, Inc.

The anti-HER2 antibody was from Sigma. Anti-phospho-ERK 1/2 and phospho-Akt were from Cell Signaling, Inc. The anti-RasGAP antibody was from BD Biosciences.

\subsection{2) Purification of recombinant GST fusion proteins}

The method used for expressing and purifying the GST fusion of the PTP domain of SHP1 and SHP2 has been described previously (25). To summarize, E. coli expressing the plasmid pGEX with either SHP2 or SHP1 were grown overnight in $100 \mathrm{~mL}$ luria bertani (LB) broth. Then the culture was diluted 1:5 with LB and was stimulated with $1 \mathrm{mM} \mathrm{IPTG} \mathrm{for} \mathrm{approximately} 3 \mathrm{~h}$. The bacteria were harvested by centrifugation before lysis in a buffer containing $20 \mathrm{mM}$ 
HEPES, $120 \mathrm{mM} \mathrm{NaCl}, 10 \%$ glycerol, $0.5 \% \mathrm{NP}-40$, and 2 mM EDTA. Sonication was used to assist the lysis, and then the lysate was incubated for 30 minutes with Triton X-100 before being cleared by centrifugation. The supernatant was applied to a slurry of glutathione sepharose beads overnight at $4^{\circ} \mathrm{C}$. The beads were washed with lysis buffer three times, and the protein was eluted using reduced glutathione $(10 \mathrm{mM})$ in tris buffer. The eluted product was collected and stored in $-80 \mathrm{oC}$ with $10 \mathrm{mM}$ dithiothreitol. The Bradford assay (Biorad, City, State) was used to quantify the product.

\subsection{3) In vitro phosphatase activity assay}

Purified SHP2 or SHP1 lacking the regulatory SH2 domains was diluted to a concentration of 5 nM with phosphatase reaction buffer (50mM HEPES, $100 \mathrm{mM} \mathrm{NaCl}$, and $2 \mathrm{mM}$ EDTA, pH=7.2). Difluoromethylumbelliferryl phosphate was used as an artificial substrate at a concentration of $20 \mathrm{uM}$ or $35 \mathrm{uM}$, corresponding to the Km for SHP2 and SHP1, respectively. Varying concentrations of peptide (sequence DADEpYL corresponding to the substrate phospho-Y992 of EGFR) were used to assess which concentration resulted in 50\% inhibition. Graphpad Prism 5 was used to calculate this IC50 value. Ki was calculated based on the Cheng-Prusoff equation:

$$
\mathrm{IC} 50=\mathrm{Ki}(1+[\mathrm{S}] / \mathrm{Km})
$$

\subsection{4) Site-directed mutagenesis, subcloning, and expression of HER2}

HER2 cDNA in a pCMV-SPORT6 vector, purchased from ATCC, was used as the template for site-directed mutagenesis using a Stratagene kit (600250). The sense and the antisense primers for the E1021A substitution were 5'- 
GGACCTGGTGGATGCTGCGGAGTATCTGGTACCCCAGCAG-3' and 5'-

CTGCTGGGGTACCAGATACTCCGCAGCATCCACCAGGTCC-3'. Both the wildtype and mutant cDNAs were then ligated into the REBNA/IRES/GFP viral vector reported previously at XhoI and NotI sites using the forward primer containing a Sall site (5'-

TTTGTCGACCGCCACCATCGCAGCT-3') and a reverse primer containing a NotI site (5'TTTTGCGGCCGCCTATTTATCATCATCAT-3') (25). Retroviruses were generated, and MCF-10A cells were infected to stably express the wildtype and HER2 mutant as described previously using blasticidine selection (1 $\mu \mathrm{g} / \mathrm{mL})(25)$.

\subsection{5) Preparation of cell lysates, immunoprecipitation, and immunostaining}

Cells were lysed in a buffer containing $20 \mathrm{mM}$ Tris-HCl (pH 7.4), $150 \mathrm{mM} \mathrm{NaCl}, 1 \mathrm{mM}$ EDTA, 1\% Triton-X-100, 10\% glycerol, and $50 \mathrm{mM} \mathrm{NaF}$ supplemented with $10 \mu \mathrm{g} / \mathrm{mL}$ each of aprotinin, leupeptin, and phenylmethylsulfonylfluoride (PMSF) for inhibition of proteases, and $10 \mathrm{mM}$ sodium orthovanadate for inhibition of phosphatases. For immunoprecipitation, the lysates were cleared by centrifugation at $12,000 \mathrm{rpm}$, incubated overnight with a primary antibody, and then precipitated using protein G sepharose beads. The beads were washed 3 times with cell lysis buffer and denatured by boiling with Laemmli sample buffer. The same denaturation procedure was used for analyzing total cell lysates. Denatured proteins were separated on $8 \%$ or $10 \%$ PAGE, immobilized onto a nitrocellulose membrane, blocked with $3 \%$ bovine serum albumin (BSA) in tris-buffered saline containing 1\% Tween-20 (TBST), and stained with a primary antibody overnight at $4^{\circ} \mathrm{C}$. Next, membranes were washed three times with TBST, incubated with horseradish peroxidase-conjugated secondary antibodies in $5 \%$ milk, washed three times with TBST, and visualized by the chemiluminescence method (Pierce Inc.). 


\subsection{6) Affinity precipitation}

The GST fusion of the PTP domain of doubly-mutated SHP2 (D425A and C459S, DM-SHP2) was used to affinity precipitate the wildtype and mutant HER2 as described previously (25). Briefly, cells at $80 \%$ confluence were incubated with $1 \mathrm{mM}$ vanadate for 30 minutes before stimulating with EGF (100 ng/mL) for 10 minutes. Lysates were then incubated with purified GST-DM-SHP2 overnight at $+4^{\circ} \mathrm{C}$, and the resulting SHP2-substrate complexes were captured by incubating the mixture with glutathione sepharose beads for two hours at $+4^{\circ} \mathrm{C}$. The beads were then washed four times with lysis buffer before adding laemmli sample buffer and boiling. The denatured samples were then subjected to separation by SDS-PAGE and analysis by immunostaining.

\subsection{7) Anchorage-independent growth assay}

The soft agar assay was performed as described previously (84). Briefly, $6 \mathrm{~cm}$ cell culture plates were coated with $0.5 \%$ agar in growth medium. Approximately $1 \times 10^{5}$ cells were suspended in growth medium that was mixed with melted agar to a final concentration of $0.5 \%$

and were then poured onto the agar bed. After four weeks of incubation a minimum of 10 fields at a $4 \mathrm{x}$ objective per plate were analyzed. Phase contrast pictures were taken using an Olympus IX71 microscope equipped with Olympus DP30BW digital camera, and colony size was estimated as a circular area using the measurement tools in Microsuite Basic Edition. Error bars represent the standard error of mean (SEM) of replicates. 


\subsection{8) Molecular docking}

was built in InsightII (Accelrys, Inc.) and saved as a pdb. The docking pre-processing and data analysis were performed in Autodock Tools (191) and the grid and docking calculations were carried out by Autogrid4 and Autodock4 (192). Nonpolar hydrogens were removed from the peptide, and Kollman charges were added. The relaxed enzyme pdb was then loaded, and an 80x80x80 grid box with 0.375 angstrom grid spacing was placed around the point defined by the sulfur of the catalytic cysteine offset in the $\mathrm{x}$ direction by 16 angstroms. Electrostatic maps for the ligand were calculated based on this grid, and then the genetic algorithm was applied to search for the best predicted binding structure of the peptide. In each case a minimum of 2000 runs were attempted. Default parameters were used for the genetic algorithm with a few exceptions. First, population size was increased to 400 , and the number of energy evaluations was set at a high number to allow for the maximum number of generations, which was set at 27000. Autodock Tools was then used to identify the best predicted binding pose, and pictures and hydrogen bonding information were gathered.

\section{4) Results}

\subsection{1) A substrate-derived peptide is a selective SHP2 inhibitor}

Previous reports showing discrimination by SHP2 of specific phosphotyrosine sites within the same protein (i.e., EGFR (49), HER2 (25), and FAK (168)) suggest that selectivity for substrates can be mediated by elements of the sequence surrounding the phosphotyrosine, as has been examined using library methods (193). Interestingly, when the primary sequences of the substrate and non-substrate phosphotyrosines were aligned, the presence of acidic amino 
acids seemed to be the most common trend. This was especially noted for the -2 position, which is the only residue where the acidic amino acid is fully conserved and exclusive to substrates (Figure 1A).

These observations led us to speculate that a phosphopeptide derived from the substrate sequence may act as a competitor to phosphatase activity. Since the endogenous substrate is thought to be selective for SHP2 as opposed to SHP1 $(194,195)$, we hypothesized that the competition using this molecule would be similarly selective. A hexapeptide was synthesized to include the four amino acids $\mathrm{N}$-terminal to the phosphotyrosine, since this region contained the highest amount of conserved acidic amino acids. The GST fusion of the PTP domains of SHP2 and SHP1 were purified as described in the materials and methods and used for in vitro phosphatase assays (196). The enzymatic activity of SHP2 and SHP1 toward the artificial substrate difluoromethylumbelliferryl phosphate (DiFMUP) was determined in the presence of different peptide concentrations. We determined the inhibitory constant, Ki, for the peptide against both enzymes, resulting in more competition for SHP2 compared with SHP1 (approximately $11.97 \mu \mathrm{M}$ SHP2 vs $630.0 \mu \mathrm{M}$ for SHP1) (Figure 1B). These data support the hypothesis that a substrate-derived inhibitor of SHP2 does not readily inhibit SHP1, and selectivity for this inhibitor is based on the amino acids surrounding the phosphotyrosine.

Since the acidic amino acid located at the -2 position in the substrate sequence was well-conserved and exclusive to substrate phosphotyrosine sequences (Figure 1A), a modified peptide was synthesized substituting the -2 position aspartate for alanine, removing the associated negative charge. This substitution effectively eliminated the inhibitory ability of the peptide when subjected to phosphatase assays against SHP2 (Ki = 856.8 $\mu \mathrm{M}$, Figure 1B). These 
results suggest that the conserved -2 position amino acid of the substrate is especially important for binding to SHP2.

\subsection{2) Mutation of HER2 abolishes interaction with SHP2}

Intracellular association of SHP2 with its substrates has been well established using the substrate-trapping, phosphatase-dead SHP2 mutant (DM-SHP2) $(25,49,197)$. Since we observed that mutation in the substrate-derived peptide sequence was able to abrogate inhibition of the enzyme, we wanted to assess whether similar mutations in a full-length substrate could prevent its association with SHP2.

Using site-directed mutagenesis, we introduced an aspartate-to-alanine substitution in the HER2 protein at position 1021, corresponding to the -2 position aspartate in the substratederived peptide. Using a retroviral vector, the mutant or wildtype HER2 was expressed in the immortalized non-transformed breast epithelial cell line MCF-10A. Next, the cells were grown to near confluence before overnight starvation followed by vanadate treatment and EGF stimulation to enhance phosphorylation of HER2. Lysates were incubated overnight with a GST fusion of the DM-SHP2 PTP domain, and the resulting complexes were captured using glutathione sepharose beads. Wildtype HER2 was able to bind SHP2 in this context, whereas the Y1023F mutant HER2 was unable to form a complex with SHP2, as described previously (25). However, the D1021A mutant HER2 was unable to form a complex with SHP2 (Figure 2A). This suggested either the D1021A mutant of HER2 was poorly phosphorylated due to altered recognition by a kinase domain, or the mutation abolished the binding capacity for SHP2. 
Antibodies selective for phosphorylated Y1023 have yet to be developed, so direct testing of the phosphorylation status of this residue would prove challenging. It has been demonstrated, however, that phosphotyrosine 1023 acts as a major docking site for the SH2 domain of p120 RasGAP (25), and if the phosphorylation status is intact, binding to RasGAP should be preserved. To this end, we immunoprecipitated RasGAP out of EGF-stimulated MCF10A cells expressing the HER2 proteins and probed for HER2 (Figure 2B). Both the wildtype and D1021A HER2 immunoprecipitated with RasGAP, while the Y1023F mutant did not to a significant extent. This suggests that the phosphorylation of D1021A HER2 at Y1023 was unaffected by the amino acid substitution, leading to the conclusion that the defective association with SHP2 is due to the removal of the acidic amino acid N-terminal to the phosphotyrosine.

\subsection{3) Disruption of HER2-SHP2 interaction reduces transformation by HER2}

It has been suggested that wildtype SHP2 promotes mitogenic signaling through HER2 by dephosphorylating tyrosine 1023 and preventing the association of RasGAP (25). We sought to determine the consequence of disrupting the SHP2-HER2 interaction while leaving the HER2RasGAP interaction in place. To test this, we assessed EGF-induced signaling in cells expressing wildtype and D1021A HER2. Overexpression of wildtype HER2 resulted in prolonged Ras/MAPK and Akt signaling compared with control cells (25) (Figure 3A). However, when D1021A HER2 is overexpressed instead, the signal was still prolonged compared with controls, but it was not as robust as in cells overexpressing the wildtype HER2.

If the MAPK and Akt signaling cascades downstream of the HER2-SHP2 interaction were disrupted by mutation of HER2, then it should be expected that transformation by HER2 
would be affected, as well. HER2 has been previously shown to promote transformation of MCF-10A cells as assessed by the soft agar assay, a measure of anchorage-independent growth (25). Therefore, we determined the effect of overexpressing mutant HER2 on formation of colonies in soft agar. As expected, wildtype HER2 formed significantly larger colonies compared with controls. However, when the D1021A mutant was overexpressed, the colonies were nearly 50\% smaller on average compared with WT HER2-overexpressing cells (Figure 3B). These colonies were significantly larger than control, suggesting that the mutant HER2 maintained partial transformative capability. These observations in soft agar corresponded with those seen in the EGF-induced signaling cascades, where D1021A attenuated but did not eliminate the advantage conferred by overexpressing wildtype HER2 (Figure 3A). It should be noted that the MCF-10A cells are grown in cell media specifically supplemented with EGF, and this media was changed once every two days. Overall, these results support the hypothesis that SHP2 promotes HER2 signaling by dephosphorylating pY1023 following EGF stimulation, preventing recruitment of RasGAP and subsequent attenuation of the MAPK signaling cascade.

\subsection{4) Molecular modeling predicts binding determinants of SHP2 and substrate}

The results described so far suggested that binding of substrate by SHP2 is supported by negatively-charged amino acids upstream of the phosphotyrosine. Indeed, the region surrounding the active site of SHP2 is highly-positively charged, moreso in general than its homologue SHP1 (198). In order to assess how the substrate-based peptide binds to SHP2 selectively, we performed molecular docking studies using Autodock to predict binding of the peptide used in Figure 1 to the SHP1 and SHP2 active sites. The DADEpYL peptide was docked into the SHP2 active site of 2SHP.pdb (with SH2 domains stripped from the structure) using the genetic algorithm. At least 2000 runs were performed, and the most favorable 
conformation was selected to predict the interaction (Figure 4). We ensured that the phosphotyrosine was situated inside the active site near the nucleophilic cysteine, an essential step of the PTP catalytic mechanism (78). In these data, actual amino acid numbers in the SHP2 protein were used to refer to residues in the active site, while the position in reference to the pY was used to assign numbers to the residues in the peptide. Accordingly, residues $\mathrm{N}$-terminal to the pY were assigned with negative numbers while those positioned C-terminal to the $\mathrm{pY}$ were given positive numbers.

Electrostatic interactions were computed in Autodock tools to estimate binding energies. Strong ionic contacts were found between arginine $(R)$ and lysine $(K)$ residues of the SHP2 active site and the acidic residues of the substrate peptide (Table 1), with the most prominent being between $\mathrm{K} 364$ and the aspartate residue at the -2 position $(-6.737 \mathrm{kcal} / \mathrm{mol})$. Other strong predicted interactions included two contacts between R362 and the aspartate in the -4 position of the peptide $(-2.827$ and $-3.653 \mathrm{kcal} / \mathrm{mol})$. The -2 aspartate also interacted with $\mathrm{K} 366(-0.113 \mathrm{kcal} / \mathrm{mol})$. Finally, the -1 glutamate residue of the peptide was predicted to hydrogen bond with Y279 of SHP2 $(-2.903 \mathrm{kcal} / \mathrm{mol})$. The total intermolecular interaction energy was found to be $-14.3 \mathrm{kcal} / \mathrm{mol}$ with $-8.50 \mathrm{kcal} / \mathrm{mol}$ originating from Van der Waals interactions and hydrogen bonds and $-5.80 \mathrm{kcal} / \mathrm{mol}$ provided by electrostatic interactions. We also calculated the predicted Ki of the peptide, which was found to be $9.72 \mu \mathrm{M}$.

To obtain further insight into the active site differences, the same peptide was docked into the SHP1 active site (Figure 5). Out of more than 2000 runs, the best structure predicted a Ki of $641 \mu \mathrm{M}$, a rather dramatic loss of predicted inhibition compared to SHP2 (9.72 $\mu \mathrm{M})$. Analysis of the structure revealed many electrostatic contacts between the peptide and the 
active site (Table 2). R358, which is analogous to K364 of SHP2, was predicted to form an ionic bond with the -2 position aspartate of the peptide $(-4.518 \mathrm{kcal} / \mathrm{mol})$. Another electrostatic interaction was found between $\mathrm{H} 420$ and the -1 glutamate of the peptide $(-3.67 \mathrm{kcal} / \mathrm{mol})$. The geometry of the predicted interactions was influenced by steric clashes. The orientation and size of R358 appeared to create a pocket that required deformation of the peptide and atom contacts with the active site, resulting in a lowered overall binding energy.

\section{5) Discussion}

This work demonstrates for the first time that a PTP substrate-based inhibitor can act as a selective antagonist of SHP2 activity compared to SHP1, a closely-related PTP family member. Here we have shown that critical interactions occur between active site basic residues and acidic residues of the peptide substrate.

Our initial observation that the substrate-derived peptide acted as a selective antagonist of SHP2 activity led to the idea that negatively-charged sidechains must play a critical role in SHP2 recognition of substrates, an idea that has been suggested by numerous studies $(185,186,190,199)$. We have demonstrated for the first time, however, the critical importance of acidic amino acids to binding, since removal of even one of these residues (the -2 position aspartate) abolishes competition for substrate in the in vitro experiments (Figure 1B). We took this observation one step further, expressing the substitution in a cellular context with a bona fide SHP2 substrate, HER2. This mutant HER2 failed to associate with SHP2 via affinity purification (Figure 2A), but its ability to coprecipitate with RasGAP indicates that the Y1023 residue is still phosphorylated despite the mutation (Figure 2B). This suggests that recognition of negatively-charged sidechains is important for SHP2 substrate determination in vivo as well as in vitro. 
Interestingly, the lost association correlated with a loss of signaling longevity in response to EGF (Figure 3A), presumably due to preserved binding of the inhibitor RasGAP. This is in agreement with the model for SHP2 mediation of HER2 signaling that was proposed previously (25). This signaling defect carried over to a defect in transformative capacity of HER2 in an anchorage-independent growth assay, further indicating a survival promoting role of the SHP2-HER2 interaction (Figure 3B).

Molecular docking was used as a tool to predict how selective binding is achieved. Specifically, electrostatic interactions of substrate acidic sidechain-containing amino acids with active site arginine 362 as well as lysine 364 (substituted to lysine and arginine in SHP1, respectively) were found to strongly mediate the binding. In addition, the difference in size of these amino acids resulted in unfavorable steric interactions when docked to SHP1 but not SHP2. We found that interactions with K364 of SHP2 conferred a significant amount of electrostatic stability, especially to the -2 position aspartate residue of the DADEpYL peptide inhibitor. We experimentally confirmed that this -2 position acidic amino acid was important for binding in vitro, in support of our computational docking results. The importance of the substrate's negatively-charged sidechains binding to the region containing R362 and K364 suggested that selectivity for SHP2 is derived primarily from distinct characteristics of this region. Interestingly, the first known SHP2 inhibitor, NSC-87877, was shown to be nonselective between SHP2 and SHP1, and docking in that study revealed that the positivelycharged region of the active site was not engaged by this compound at all, which may help to explain its lack of selectivity for SHP2 over SHP1 (183).

The differences in the predicted energies of polar contacts revealed special importance of sidechain interactions to the binding of acidic residue-containing peptides to SHP2, but not 
necessarily SHP1. Side chain interactions between the peptide's acidic residues and the active site resulted in a total energetic stabilization twice as large for SHP2 $(-16.238 \mathrm{kcal} / \mathrm{mol})$ as for SHP1 (-8.756 kcal $/ \mathrm{mol})$. This may explain why SHP2 is able to bind and selectively dephosphorylate EGFR pY992, while SHP1 does not. It also supports the idea that SHP1 and SHP2 have divergent active site selectivity determinants, and this selectivity is not due to trafficking by their unique SH2 domains (200).

In conclusion, we have presented data elucidating specific substrate-SHP2 intermolecular interactions that are present during binding of a substrate-derived phosphopeptide inhibitor of SHP2. Interactions with a positively-charged region of the active site that are conserved in sequence, but not structure between SHP2 and SHP1, are found to be present and appear to be critical for inhibition as determined by in vitro assays. This substrate-derived peptide shows substantial selectivity for SHP2 over SHP1, an improvement over currently-published inhibitors. While we do not expect this peptide to be an acceptable therapeutic agent in itself, we expect that the knowledge of selectivity determination will be useful in the development of improved small molecule inhibitors.

\section{6) Figure Legends}

Figure 1. a) The major phosphotyrosine sites in EGFR and HER2 were overlaid for comparison; common features to SHP2 substrate sequences were analyzed, and a substrate-derived peptide was synthesized. b) Activity of the substrate-derived peptide was assessed in competition with the fluorogenic difluoromethylumbelliferryl phosphate in increasing concentrations to arrive at an IC50 as described in Materials and Methods. The modified substrate, with the -2 position aspartate substituted, showed no activity toward SHP2. 
Figure 2. a) Whole cell extracts of MCF-10A cells expressing either vector control, WT-HER2, 1023F-HER2, or 1021A-HER2 following EGF stimulation (25).

b) MCF-10A cells expressing wildtype or mutant HER2 were stimulated with EGF for 10 minutes, and lysates were subjected to immunoprecipitation with RasGAP to determine phosphorylation status of Y1023.

c) Whole-cell extracts of MCF-10A cells described in "a" were incubated overnight in the presence of GST-DM-SHP2 and glutathione-sepharose beads. Captured complexes were washed four times and then were immunoblotted for HER2.

Figure 3. a) MCF-10A cells overexpressing wildtype or mutant HER2 were stimulated with EGF $(100 \mathrm{ng} / \mathrm{mL})$ over the given time points. Lysates were subjected to SDS-PAGE and immunoblotting.

b) MCF-10A cells were subjected to an anchorage-independent growth assay as described previously (84). Briefly, $1 \times 10^{\wedge} 5$ cells were suspended in a $0.5 \%$ agar and were allowed to grow, refreshing media every two days. Colony size was estimated as a circular area based on 10 different fields of colonies per replicate. Data are presented as mean \pm SEM. ${ }^{* * *}, \mathrm{p}<0.001$

Figure 4. a) EGFR Y992-derived peptide was docked into the SHP2 active site using Autodock4, and the binding mode is presented with SHP2 residues represented using the molecular surface model.

b) Interactions between the peptide and the SHP2 active sites. Peptide residues are labeled based on their sequence position relative to the phosphotyrosine. Polar contacts are indicated by green dots. 
Figure 5. a) EGFR Y992-derived peptide was docked into the SHP1 active site using Autodock4, and the binding mode is presented with SHP1 residues represented using the molecular surface model.

b) Interactions between the peptide and the SHP1 active sites. Peptide residues are labeled based on their sequence position relative to the phosphotyrosine. Polar contacts are indicated by green dots. 
Figure 1. Derivation of a substrate-derived peptide that selectively inhibits SHP2
A)
EGFR
DVVDADEY ${ }_{992}$ LIPQQG
TFLPVPEY 1068 INQSVP
GSVQNPVY 1086 HNQPLN
ISLDNPDY $_{1148}$ QQDFFP
STAENAEY 1173 LRVAPQ
HER2
DLVDAEEY $_{1023}$ LVPQQG
HDPSPLQY 1112 SEDPTV
TCSPQPEY $_{1139}$ VNQPDV
GAVENPEY 1196 LTPQGG
PAFDNLYY 1222 WDQDPP
AVAENPEY $_{1248}$ LGLDVP

B )

\begin{tabular}{|lc|}
\hline \multicolumn{2}{|l|}{ Testing wildtype peptide } \\
\hline DADEpYL Experimental Ki $(\mu \mathrm{M})$ \\
\\
\hline SHP1 \\
SHP2 & 630.0 \\
\hline Testing mutant peptide \\
\hline DAAEpYL \\
\hline SHP2 & 11.97 \\
\hline
\end{tabular}


Figure 2. Mutation of HER2 abolishes interaction with SHP2

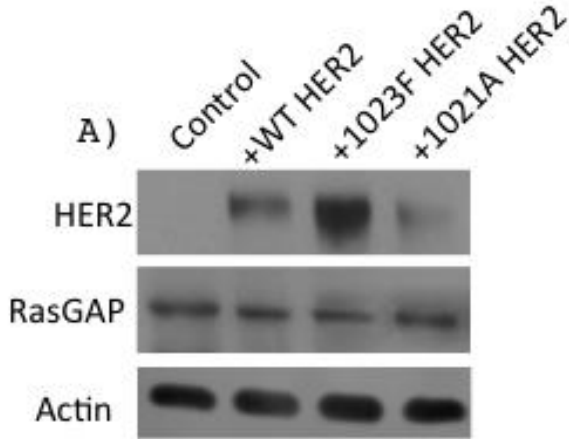

Input TCL

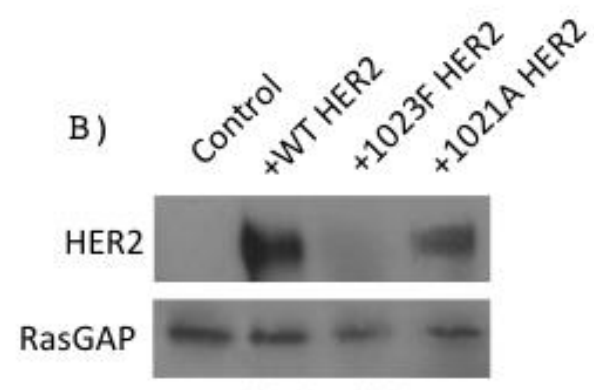

IP: RasGAP

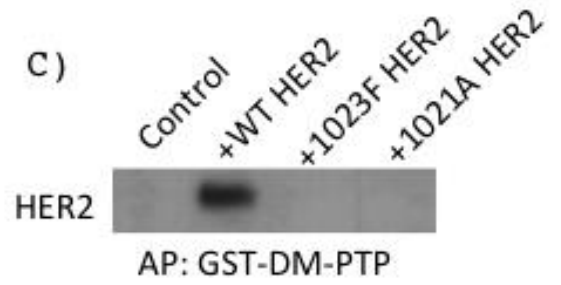


Figure 3. Mutation of HER2 results in attenuated response to EGF stimulation and inhibits transformation

A)

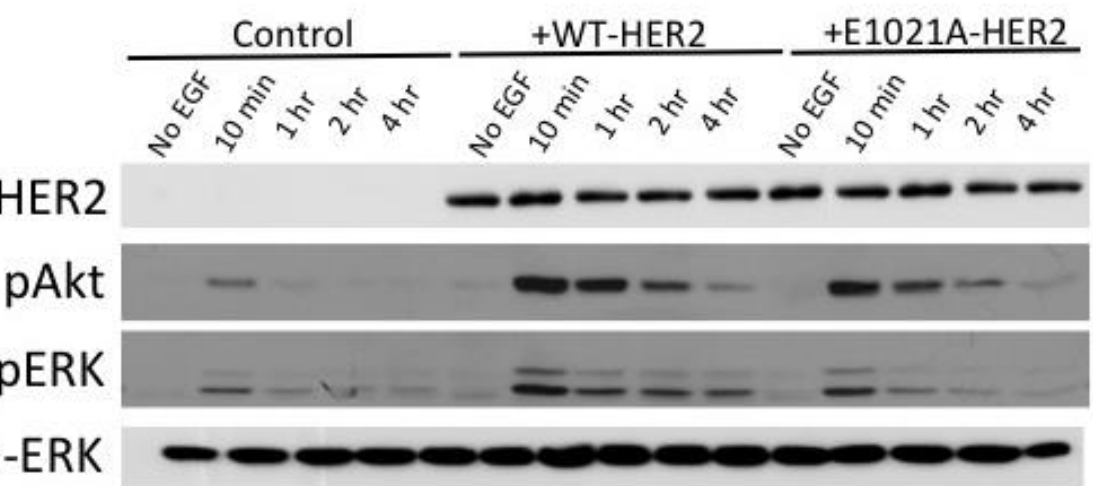

B)

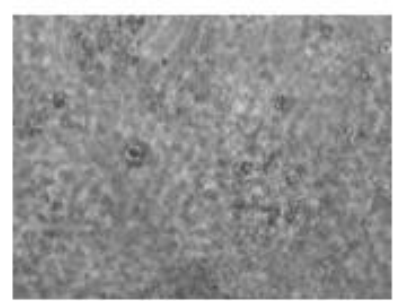

Control

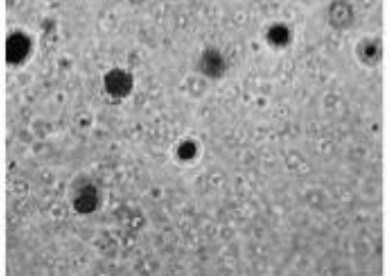

+WT HER2

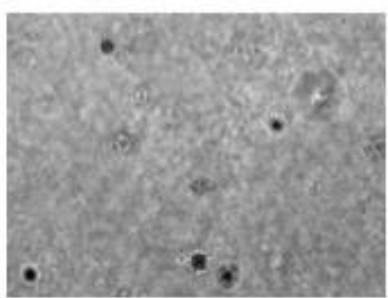

$+1021 \mathrm{~A}$ HER2

Soft Agar Assay, MCF-10A

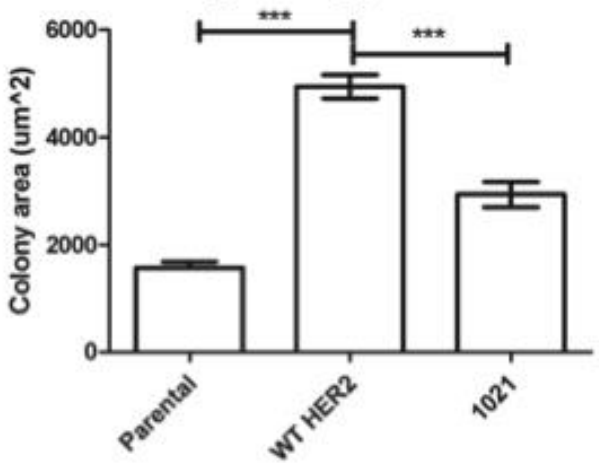


Figure 4. Predicted binding mode of substrate-derived peptide to the SHP2 active site

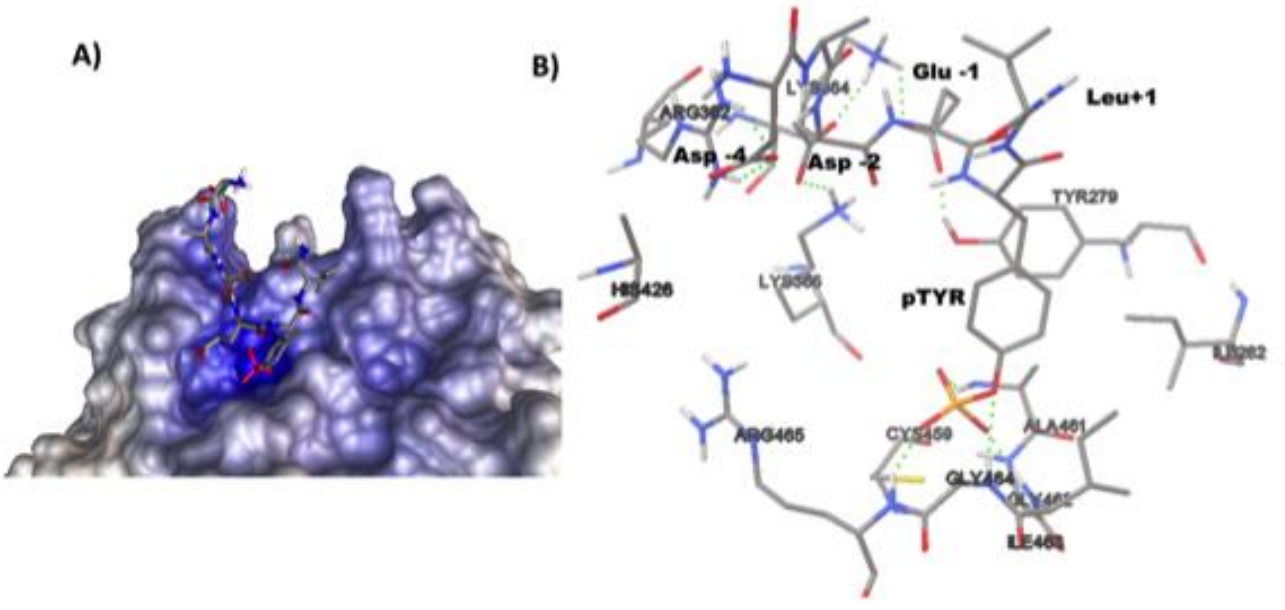


Figure 5. Predicted binding mode of substrate-derived peptide to the SHP1 active site

A)

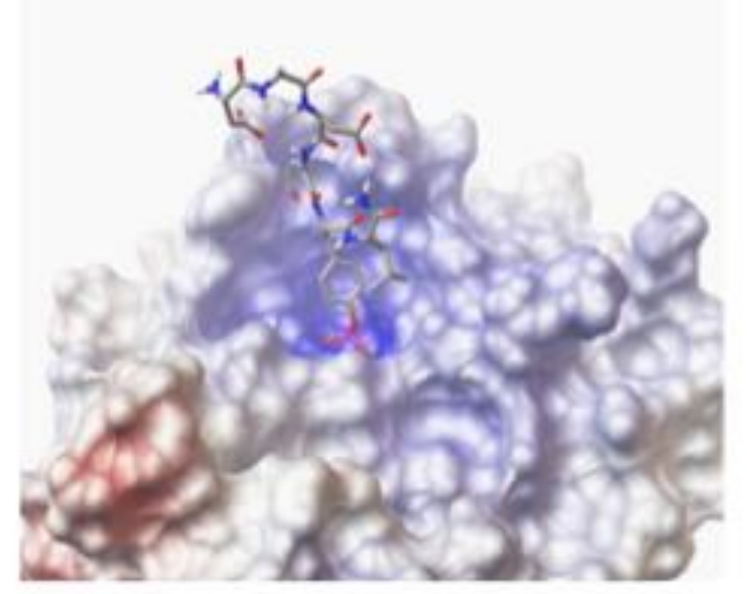

B)

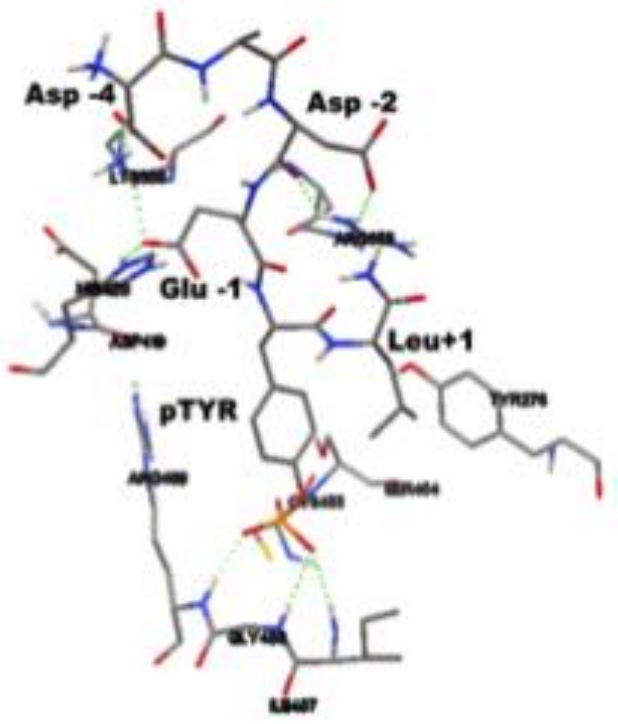


Table 1. Predicted electrostatic interactions between the substrate-derived peptide and the SHP2 active site

\begin{tabular}{|ll|}
\hline Peptide to SHP2 & Energy* \\
\hline -1 Glu to Y279 & -2.903 \\
-2 Asp toK364 & -6.737 \\
-2 Asp to K366 & -0.113 \\
-4 Asp to R362 & -2.827 \\
-4 Asp to R362 & -3.653 \\
\hline
\end{tabular}

*Energy given in $\mathrm{kcal} / \mathrm{mol}$ 
Table 2. Predicted electrostatic interactions between the substrate-derived peptide and the SHP1 active site

\begin{tabular}{|ll|}
\hline Peptide to SHP1 & Energy* \\
\hline -1 Glu to H420 & -3.67 \\
-1 Glu to K356 & -0.301 \\
-2 Asp to R358 & -4.518 \\
-2 Asp to R358 & -0.26 \\
-4 Asp to K356 & -0.007 \\
\hline
\end{tabular}

*Energy given in $\mathrm{kcal} / \mathrm{mol}$ 


\section{6) Discussion}

The overall goal of the work presented in this report is to add to the growing body of evidence supporting SHP2 as a therapeutic target in breast cancer. At this time, more than half of all oncology drug development focuses on dissecting and targeting tyrosine kinases (30). Many of the seminal discoveries in targeted therapy have been made with respect to tyrosine kinases, including the development of imatinib (targeting Bcr/Abl) and trastuzumab (targeting HER2), among others. Unfortunately, these research efforts, while representing a paradigm shift in cancer therapy, have yet to achieve the ultimate goal: eradicating cancer. Therefore, the search continues for novel drug targets.

Our study into protection of EGFR by HER2 illustrates an important trait of breast cancer, flexibility. Targeting of one pathway-in this case, HER2 signaling-- via upstream inhibition has been problematic since the introduction of trastuzumab. Up to half the patients with HER2-positive disease fail to respond to this therapy at all (201). A combined approach to breast cancer therapy is clearly needed. These combination therapies, however, have been slow to evolve. Many questions arise with this strategy. First, does combination therapy improve outcome? Several large studies have examined targeting receptor tyrosine kinases at multiple points. For example, combination lapatinib (a small molecule HER2 kinase inhibitor) plus trastuzumab led to an overall improved response to therapy when compared with either agent alone (202). Second, is combination therapy more toxic? Additive toxicity has been a significant challenge so far in combination therapy. Lapatinib plus trastuzumab leads to severe diarrhea and liver enzyme changes, but these effects were manageable in the trial (202). 
Toxicity profile changes can become so severe, however, that the trial must be terminated, as was the case with combination bevacizumab (an antibody that scavenges vascular endothelial growth factor, VEGF) and sorafenib (a small molecule VEGFR kinase inhibitor) (203). In all, it is clear that multi-agent therapy creates new challenges. If these initiatives fail, scientists lose years of effort.

As of today, targeted breast cancer therapy focuses mainly on the upstream mediators of signaling. Many agents act either at the level of the extracellular domain or the kinase domain of receptor tyrosine kinases. While these drugs have improved the lives of many patients, disease relapse is a common outcome. One reason for this phenomenon is the ability of cancer cells to recruit redundant pathways to promote survival and growth. Indeed, the Cancer Genome Atlas project results illustrate just how complex breast cancers can become (22). Many tumors with HER2-positive or triple-negative phenotypes show activation of seemingly-unrelated receptor tyrosine kinases (i.e., fibroblast growth factor receptor). Thus, even with multiple agents, targeting the upstream mediators of signaling is challenging.

To date, cancer drug trials have not targeted downstream integrators of signaling. Previous work has demonstrated the importance of SHP2 in the proliferation and survival of cancer cells, helping to define SHP2 as a bona fide proto-oncogene (204). Increasing its activity can contribute to breast cancer tumorigenesis and progression $(27,28,84)$. SHP2 promotes the activity of every known breast cancer-related receptor tyrosine kinase pathway, including EGFR and FGFR family members, to name a few. Transgenic mouse models and xenografts (27) have established key pre-clinical data showing that SHP2 is a critical mediator of breast tumorigenesis and maintenance. 
Further support for targeting SHP2 comes from multiple studies analyzing breast cancer patient samples. SHP2 overexpression correlates with higher tumor grade and worse outcome $(27,28,85)$. The first paper in this line demonstrated an association between SHP2positivity and lymph node metastasis, a feature of advancing carcinoma that often portends the development of incurable, metastatic cancer (28).

Targeting of SHP2 has the added advantage of being impervious to many of the resistance mechanisms that challenge inhibition of receptor tyrosine kinases. For example, HER2-positive disease can overcome trastuzumab therapy via ectodomain shedding or through activation of growth factor pathways. Small molecule inhibitors of the HER2 kinase domain can be resisted through mutations that prevent binding of the small molecule while retaining ATP-binding capacity. An inhibitor targeting SHP2 would face neither of these challenges, since 1) it possesses no targeted domain it can shed to avoid binding an inhibitor and 2) any mutation that would abolish binding to a substrate-mimicking inhibitor would likely inhibit binding to endogenous substrates, as well, suppressing SHP2's function.

The other two studies presented in this paper address two differing aspects of SHP2 biology and the hypothesis that SHP2 is a useful therapeutic target in breast cancer. From these studies, we can make two major conclusions: 1) SHP2 controls an important component of metastasis in breast cancer cells, and 2) Design of selective SHP2 inhibitors is possible.

\section{1) SHP2 controls one component of metastasis in breast cancer cells}

In line with the correlation between overexpressed SHP2 and metastasis, we have published results indicating the importance of SHP2 in wound healing of triple-negative breast cancer 
cells. Wound healing is analogous to tumor cell movement along tracks of tissue or bone in vivo, a component of metastasis (205). SHP2 is an important regulator of adhesion and motility signaling (64), but a clear picture of the mechanisms for this control has remained elusive. We presented live-cell data demonstrating that SHP2 controls growth factor-stimulated lamellipodial dynamics. Specifically, inhibition of SHP2 hinders persistence of these structures, which may account for the some of the overall defect of these cells to move in response to EGF stimulation.

The formation and maturation of nascent adhesions following protrusion is one important constituent of lamellipodial persistence (206). SHP2 was previously shown to control adhesion strengthening of cells in other contexts (67). In agreement with von Wichert, et al., we found that inhibition of SHP2 upregulated FAK activation, which enhances focal adhesion dynamics and lower adhesion strengthening (67). In our study, the mechanism of persistence and a role for FAK activity downregulation were not clear from our data alone. However, other lines of evidence point to several mediators. One of these is $\alpha$-actinin, an actinbinding protein that becomes phosphorylated by FAK and stabilizes stress fibers. When SHP2 is inhibited, the phosphorylation of $\alpha$-actinin rises, as well, leading to aberrant stress fiber formation and enhanced cell contractility, weakening adhesions (67).

A more speculative hypothesis of nascent adhesion control involves the phosphoprotein Nudel, which has never been experimentally established to function alongside SHP2. Despite this lack of evidence, Nudel possesses interesting functions that relate to our study. First, it is trafficked specifically to nascent adhesions, where it helps to promote their strength. It accomplishes this feat, at least in part, by binding paxillin, though the full mechanism is not 
understood (207). When FAK is in its open conformation (e.g., following phosphorylation of Y397 and subsequent phosphorylation of the activation loop via recruitment of Src), it binds and sequesters paxillin, preventing binding of Nudel. In this context, SHP2, through dephosphorylation of FAK pY397, could prevent this sequestration, thus promoting nascent adhesion strength.

Regardless of the mechanism, SHP2 control of triple-negative breast cancer cell motility supports the hypothesis that this enzyme as a viable target in breast cancer. In particular, control of growth factor-related motility by SHP2 is useful in triple-negative cancer for two reasons. First, this subtype of cancer results in higher cancer-related mortality despite its relatively-low incidence (10). This is primarily due to the predisposition of triple-negative cancers to metastasize. The lack of hormone receptors and HER2 removes clinicians' only approved targeted therapies, so options are more limited for these patients. Second, triplenegative cancers often overexpress EGFR (172), so control by SHP2 presents an enticing focus for future therapy.

\section{2) Design of selective SHP2 inhibitors is possible}

Development of inhibitors against SHP2 has gained momentum in the last decade (208). The first compound developed to target SHP2 ran into the two major problems the field has encountered so far: cell permeability and selectivity (209). This compound, NSC-87877, was found using a high-throughput screen of the NCI diversity set. It demonstrated the highest potency against SHP2 of any compound to date, with an IC50 of approximately $300 \mathrm{nM}$. Unfortunately, activity against the homologue SHP1 was almost identical, demonstrating that NSC-87877 was not a selective compound. Inhibition of SHP1 in combination with SHP2 is an 
undesirable effect of therapy, since SHP1 generally plays a negative role in signaling within its milieu, the hematopoietic system. Owing to the tumor suppressor function of SHP1 (210), coinhibition with SHP2 may lead to malignancy. In addition, NSC-87877 required concentrations as high as $50 \mu \mathrm{M}$ to inhibit the MAPK pathway downstream of EGF stimulation, since the phosphomimetic moiety utilized (a sulfonate) was negatively charged at physiologic $\mathrm{pH}$, rendering the compound cell impermeable.

One of the compounds that has given the most insight and promise for targeting SHP2 is also one of the most recent. The salicylic acid-containing scaffolds yield effective SHP2 inhibitors while maintaining drug-like properties (211). The major lead compound from this work, II-B08, exhibits somewhat selective inhibition of SHP2 over SHP1 and other phosphatases (approximately 3-fold selectivity). Interestingly, this publication presented the first real structural clues about the binding mode of small molecule inhibitors to SHP2. II-B08 crystallized with the SHP2 active site and gave the first picture of inhibitor binding, with strong interactions being made to the phosphotyrosine binding pocket as well as surrounding amino acids. This crystal structure is novel due to the challenge of crystallizing any molecule with SHP2, substrate or inhibitor, to the extent that the quality of the structure presented with II-B08 is disputed (Dr. Nicholas Lawrence, personal communication).

II-B08 has had the most success in moving through pre-clinical experiments. It has been used in two different animal-based models of malignancy, including mutant c-Kit-induced myeloproliferative disorders when combined in a regimen with a PI3K inhibitor (212). II-B08 was also tested in a mouse model of non-small cell lung carcinoma (NSCLC), the most common form of lung cancer (213). Inhibition of SHP2 via a small molecule resulted in reduced EGF- 
stimulated MAPK and Akt induction; in addition, the growth of NSCLC cell xenografts was significantly suppressed in animals treated with II-B08. The cells grafted into the animals harbored a genetic mutation in the EGFR gene, conferring the classic T790M substitution that renders the protein insensitive to small molecule inhibition by kinase inhibitors such as gefitinib. While promising, the rate of metastasis of the xenografts was not explored, and the relative lack of selectivity for II-B08 still leaves a potential issue: inhibition of SHP1 in the hematopoietic compartment and possible resultant malignancy. This question was not explored in the studies published so far, and the issue of drug selectivity remains.

Many insights regarding SHP2 selectivity have been inferred by SHP2-drug interactions $(185,186,190,199) ;$ however, no lab has yet taken a substrate-based approach to deduce selective binding determinants. Our mutagenesis work represents the first demonstration of a mutated upstream recognition sequence in a substrate ablating binding of SHP2. The result was a HER2 molecule with intact RasGAP binding that was less able to promote signaling than wildtype. This HER2 mutant still promoted signaling and transformation, but it was to a lower extent compared with wildtype HER2. These results help to answer two questions that have lingered since our lab's first exploration of HER2 and SHP2 in 2009 (25):

1) Are upstream acidic amino acids important for recognition of substrates by SHP2?

At the in vitro and cellular level, we were able to clearly confirm the importance of an acidic amino acids at the -2 position of a SHP2 substrate. This observation should not be taken to mean, however, that only the -2 position is key in decision making for SHP2. The modeling data suggested that removal of any of the three upstream negatively-charged sidechains would significantly impair binding, but these other positions-- the - 1 and -4 positions of the DADEpYL 
peptide-- were not explored heavily in the study. The reason was that the -2 position was the only acidic amino acid in all of the known EGFR/HER2 autophosphorylation sites that was present in only the Y992/Y1023 sequences. Other phosphotyrosines had acidic amino acids at the -1 and/or -4 positions, making a definitive conclusion of importance to binding more difficult.

The result of this study was the discovery of a model inhibitory compound with improved selectivity for SHP2 over SHP1 compared with previously-developed small molecule inhibitors. In our data, this selectivity was above 50 -fold. If these observations can be translated into a peptidomimetic compound, then we hypothesize that selectivity of anti-SHP2 molecules can be improved dramatically.

2) Is RasGAP inhibition the major route of SHP2 enhancement of HER2 signaling?

Our lab's study of SHP2 regulation of HER2 signaling and transformation demonstrated several important findings (25). First, SHP2 recognized only the pY1023 site of the protein. Mutation of other candidate phosphotyrosines had little to no effect on co-precipitation with SHP2. Second, mutation of Y1023 to phenylalanine ablated the binding of both SHP2 and RasGAP, strongly promoting downstream signaling. This suggests a role for SHP2 as the inhibitor of the inhibitor in this context; however, SHP2 has been shown to control receptor tyrosine kinase signaling through other pathways, as well (78). In addition, total ablation of RasGAP binding to HER2 alone does not prove that inhibition of its recruitment by SHP2 is the major mechanism for promoting transformation. Introduction of a D1021A mutation appeared to have no effect on the recruitment of RasGAP. This was expected since the binding of SH2 domains is known to proceed primarily through sequences $\mathrm{N}$-terminal to phosphotyrosines. The resulting inhibited 
signaling and transformation are consistent with the hypothesis that the major role of SHP2 in promoting HER2-induced signaling is dephosphorylation of pY1023 and impaired recruitment of inhibitory factors such as RasGAP.

Discrimination of substrates by SHP2 has remained a mystery to the field since SHP2 was discovered. Early studies using peptides to determine SHP2 selectivity gave some indication about residues that might be important for SHP2 to recognize and act upon a substrate, but these were not conclusive (214). A more systematic and comprehensive approach was later taken to determine selectivity using a peptide library and a novel bead assay (190). Unfortunately, this report was only able to arrive at a few conclusions. First, SHP2 preferred acidic amino acids at any position $\mathrm{N}$-terminal to the phosphotyrosine. Second, SHP2 was highly unlikely to act on a substrate peptide with a lysine or an arginine residue. Interestingly, the -2 position was most strongly predicted to be an aspartate residue, as is the case with our model substrate-based peptide. While enticing, this report did not solve the unanswered questions regarding atomic interactions needed for SHP2 binding.

Ideally, determination of substrate-active site interactions would be determined by crystallography. However, no such structure has been determined. Thus, computational modeling was a valuable predictive tool to determine the binding mode of a substrate. The finding that electrostatic interactions with R362 and K364 of the SHP2 active site would create the most stable binding is a notion that has been forwarded by other modeling studies of small molecules $(185,186,199)$. An interesting corresponding observation is the predicted binding mode of the original SHP2 inhibitor, NSC-87877 (209). When the researchers in this study docked the compound to the active site, the sulfonate interacted with the phosphotyrosine 
binding pocket as expected, but the basic region containing arginine 362 and lysine 364 were neglected entirely in favor of a hydrophilic region across from it. I speculate that the lack of selectivity of this molecule may be related to this lack of interaction with the basic region, though this has yet to be tested. Another interesting observation was noted in the paper describing the most selective SHP2 inhibitor to date, PHPS1 (199). The only residue in the basic region this molecule was predicted to engage was the arginine 362 . This was demonstrated to be important through mutation of R362, which ablated the inhibition by PHPS1. Combining these observations with our own, it seems that the most optimal selective molecule would bind the phosphotyrosine binding pocket as well as both the R362 and K364 residues in order to generate the most selectivity.

\section{3) Proposed Experiments}

The future of targeting SHP2 hinges on several unknowns. Drug design is a potentially significant hurdle. If a selective molecule cannot be developed, then the risk for unexpected adverse events becomes worrisome. This is especially true with respect to SHP2 versus SHP1 selectivity, since there is the potential for developing hematopoietic malignancy if SHP1 is inhibited (215-218). The data provided in the selectivity study may be useful in the design of an ideal inhibitor, one that can achieve many-fold selectivity over today's best small molecules. Rational design is an important factor to consider here, since drug screens conducted to find SHP2 inhibitors cannot be negatively selected at the same time for SHP1. This has resulted in troublesome compounds being discovered as leads. I envision that the data provided through molecular modeling can help the drug designers in the SHP2 field to pursue design of a peptidomimetic that is both drug-like and selective. 
Drug design issues are dwarfed by a larger unanswered question in the field: Will inhibition of SHP2 impact breast tumors? As of today, this question has not been tested directly. The most immediate experiment that needs to be performed is pharmacologic inhibition in mouse models of breast cancer. II-B08 has proof of principle established in lung cancer xenografts, suggesting this molecule is indeed bioavailable. There are many appropriate breast cancer models that can be tested in this line, including transgenic, spontaneous HER2positive tumors (driven by MMTV-Neu) and xenograft transplants of cell lines. The efficacy against metastasis by SHP2 inhibitors has yet to be evaluated in mice, as well. This should be a relatively trivial experiment, however, since tumors grown in mice are often dissected for analysis by pathological or immunohistochemical staining. Primary target sites of metastasis (e.g., lung and liver) can be excised at the same time. More to point, the tail-vein injection can be used as a direct metastasis experiment, bypassing the need to first establish a primary tumor.

Will inhibition of SHP2 impact cancer metastasis? Previous studies have suggested a correlation between metastasis to lymph nodes and overexpression of SHP2 (28). In addition, our studies into the role of SHP2 in cell motility imply that pharmacologic inhibition should suppress metastasis. This motility experiment can be expanded, however, to accommodate other stimuli of protrusions. EGF signaling is known to be important for a large subset of breast cancer, but is the defect of lamellipodial persistence we observed carried through into stimulation by other growth factors? This facet of SHP2 signaling should be explored further, since our study was the first to demonstrate a role for SHP2 in regulating dynamics of lamellipodia; however, the lack of response to EGF following SHP2 inhibition fails to account for all of the wound healing defects we observed. Regulation of FAK by SHP2 is clearly not the 
only player in this process, either, since our rescue experiment using the FAK inhibitor was unable to correct the less motile phenotype observed under normal serum conditions. Further exploration into this matter would be particularly valuable. Other experiments controlling prometastatic phenotypes controlled by SHP2 (e.g., proteolysis, 3D cell morphology) have yet to be performed in depth. If SHP2 is indeed important for metastasis, it will be critical to determine the mechanisms that promote these phenotypes.

\section{4) Conclusions}

In total, the studies presented in this thesis add incremental evidence to three facets of SHP2 in breast cancer:

1) EGFR-HER2 crosstalk is a complicating factor in therapy, and alternative strategies may be valuable for treating HER2-positive breast cancer.

2) SHP2 can promote motility of breast cancer cells by controlling FAK, providing one more line of evidence supporting the use of an anti-SHP2 approach in therapy.

3) Better selectivity can be achieved in future anti-SHP2 inhibitors, and the observations of substrate-active site interactions may lead to rational design of improved molecules.

\section{7) References}

1. Breast Cancer Key Statistics.

2. Allen, J. M. (2010) The American journal of managed care 16, 697-704

3. Wan, Y., Gao, X., Mehta, S., Wang, Z., Faria, C., and Schwartzberg, L. (2013) Journal of medical economics 16, 1169-1178

4. Pasqualini, J. (2008) Breast Cancer: Prognosis, Treatment, and Prevention, 2 ed., Informa Healthcare, New York

5. Hanahan, D., and Weinberg, R. A. (2011) Cell 144, 646-674

6. Beatson, G. (1896) The Lancet 148, 162-165 
7. Cleator, S., Heller, W., and Coombes, R. C. (2007) The lancet oncology 8, 235-244

8. Perou, C. M., Sorlie, T., Eisen, M. B., van de Rijn, M., Jeffrey, S. S., Rees, C. A., Pollack, J. R., Ross, D. T., Johnsen, H., Akslen, L. A., Fluge, O., Pergamenschikov, A., Williams, C., Zhu, S. X., Lonning, P. E., Borresen-Dale, A. L., Brown, P. O., and Botstein, D. (2000) Nature 406, 747-752

9. Parkin, D. M. (2009) European journal of cancer (Oxford, England : 1990) 45, 1649-1653

10. Gluz, O., Liedtke, C., Gottschalk, N., Pusztai, L., Nitz, U., and Harbeck, N. (2009) Annals of oncology : official journal of the European Society for Medical Oncology / ESMO 20, 1913-1927

11. Jordan, V. C. (1984) Pharmacol Rev 36, 245-276

12. Shih, C., Padhy, L. C., Murray, M., and Weinberg, R. A. (1981) Nature 290, 261-264

13. Padhy, L. C., Shih, C., Cowing, D., Finkelstein, R., and Weinberg, R. A. (1982) Cell 28, 865-871

14. Schechter, A. L., Stern, D. F., Vaidyanathan, L., Decker, S. J., Drebin, J. A., Greene, M. I., and Weinberg, R. A. (1984) Nature 312, 513-516

15. Coussens, L., Yang-Feng, T. L., Liao, Y. C., Chen, E., Gray, A., McGrath, J., Seeburg, P. H., Libermann, T. A., Schlessinger, J., Francke, U., and et al. (1985) Science 230, 1132-1139

16. Slamon, D. J., Clark, G. M., Wong, S. G., Levin, W. J., Ullrich, A., and McGuire, W. L. (1987) Science 235, 177-182

17. Cho, H. S., Mason, K., Ramyar, K. X., Stanley, A. M., Gabelli, S. B., Denney, D. W., Jr., and Leahy, D. J. (2003) Nature 421, 756-760

18. Slamon, D. J., Godolphin, W., Jones, L. A., Holt, J. A., Wong, S. G., Keith, D. E., Levin, W. J., Stuart, S. G., Udove, J., Ullrich, A., and et al. (1989) Science 244, 707-712

19. Drebin, J. A., Link, V. C., Weinberg, R. A., and Greene, M. I. (1986) Proceedings of the National Academy of Sciences of the United States of America 83, 9129-9133

20. Slamon D, L.-J. B., Shak S, et al. (1998) Proc Am Soc Clin Oncol 17

21. Markert, A., Thierry, V., Kleber, M., Behrens, M., and Engelhardt, M. (2009) International journal of cancer. Journal international du cancer 124, 722-728

22. (2012) Nature 490, 61-70

23. Liu, P. C., Liu, X., Li, Y., Covington, M., Wynn, R., Huber, R., Hillman, M., Yang, G., Ellis, D., Marando, C., Katiyar, K., Bradley, J., Abremski, K., Stow, M., Rupar, M., Zhuo, J., Li, Y. L., Lin, Q., Burns, D., Xu, M., Zhang, C., Qian, D. Q., He, C., Sharief, V., Weng, L., Agrios, C., Shi, E., Metcalf, B., Newton, R., Friedman, S., Yao, W., Scherle, P., Hollis, G., and Burn, T. C. (2006) Cancer biology \& therapy 5, 657664

24. Nahta, R., and Esteva, F. J. (2006) Cancer letters 232, 123-138

25. Zhou, X., and Agazie, Y. M. (2009) The Journal of biological chemistry 284, 12226-12234

26. Grossmann, K. S., Rosario, M., Birchmeier, C., and Birchmeier, W. (2010) Advances in cancer research $106,53-89$

27. Aceto, N., Sausgruber, N., Brinkhaus, H., Gaidatzis, D., Martiny-Baron, G., Mazzarol, G., Confalonieri, S., Quarto, M., Hu, G., Balwierz, P. J., Pachkov, M., Elledge, S. J., van Nimwegen, E., Stadler, M. B., and Bentires-Alj, M. (2012) Nat Med 18, 529-537

28. Zhou, X., Coad, J., Ducatman, B., and Agazie, Y. M. (2008) Histopathology 53, 389-402

29. Chan, R. J., and Feng, G. S. (2007) Blood 109, 862-867

30. Cohen, P., and Alessi, D. R. (2013) ACS Chem Biol 8, 96-104

31. Neel, B. G., Gu, H., and Pao, L. (2003) Trends in biochemical sciences 28, 284-293

32. Denu, J. M., and Dixon, J. E. (1998) Curr Opin Chem Biol 2, 633-641

33. Zhang, Z. Y. (2003) Progress in nucleic acid research and molecular biology 73, 171-220

34. Adachi, M., Sekiya, M., Miyachi, T., Matsuno, K., Hinoda, Y., Imai, K., and Yachi, A. (1992) FEBS Lett 314, 335-339

35. Freeman, R. M., Jr., Plutzky, J., and Neel, B. G. (1992) Proceedings of the National Academy of Sciences of the United States of America 89, 11239-11243 
36. Ahmad, S., Banville, D., Zhao, Z., Fischer, E. H., and Shen, S. H. (1993) Proceedings of the National Academy of Sciences of the United States of America 90, 2197-2201

37. Feng, G. S., Hui, C. C., and Pawson, T. (1993) Science 259, 1607-1611

38. Vogel, W., Lammers, R., Huang, J., and Ullrich, A. (1993) Science 259, 1611-1614

39. Hof, P., Pluskey, S., Dhe-Paganon, S., Eck, M. J., and Shoelson, S. E. (1998) Cell 92, 441-450

40. Ostman, A., Hellberg, C., and Bohmer, F. D. (2006) Nat Rev Cancer 6, 307-320

41. Araki, T., Nawa, H., and Neel, B. G. (2003) The Journal of biological chemistry 278, 41677-41684

42. Gutch, M. J., Flint, A. J., Keller, J., Tonks, N. K., and Hengartner, M. O. (1998) Genes Dev 12, 571-585

43. Perkins, L. A., Johnson, M. R., Melnick, M. B., and Perrimon, N. (1996) Dev Biol 180, 63-81

44. Tang, T. L., Freeman, R. M., Jr., O'Reilly, A. M., Neel, B. G., and Sokol, S. Y. (1995) Cell 80, 473-483

45. Shi, Z. Q., Lu, W., and Feng, G. S. (1998) The Journal of biological chemistry 273, 4904-4908

46. Klinghoffer, R. A., and Kazlauskas, A. (1995) The Journal of biological chemistry 270, 22208-22217

47. Cleghon, V., Feldmann, P., Ghiglione, C., Copeland, T. D., Perrimon, N., Hughes, D. A., and Morrison, D. K. (1998) Mol Cell 2, 719-727

48. Pierre, S., Bats, A. S., and Coumoul, X. (2011) Biochemical pharmacology 82, 1049-1056

49. Agazie, Y. M., and Hayman, M. J. (2003) Molecular and cellular biology 23, 7875-7886

50. Hanafusa, H., Torii, S., Yasunaga, T., Matsumoto, K., and Nishida, E. (2004) The Journal of biological chemistry 279, 22992-22995

51. Cunnick, J. M., Mei, L., Doupnik, C. A., and Wu, J. (2001) The Journal of biological chemistry 276, 24380-24387

52. Grossmann, K., Rosario, M., Birchmeier, C., and Birchmeier, W. (2010) Advances in Cancer Research $106,53-89$

53. Zhang, S. Q., Tsiaras, W. G., Araki, T., Wen, G., Minichiello, L., Klein, R., and Neel, B. G. (2002) Molecular and cellular biology 22, 4062-4072

54. Ivins Zito, C., Kontaridis, M. I., Fornaro, M., Feng, G. S., and Bennett, A. M. (2004) J Cell Physiol 199, 227-236

55. Aksamitiene, E., Kiyatkin, A., and Kholodenko, B. N. (2012) Biochemical Society transactions 40, 139146

56. Mendoza, M. C., Er, E. E., and Blenis, J. (2011) Trends in biochemical sciences 36, 320-328

57. Berchtold, S., Volarevic, S., Moriggl, R., Mercep, M., and Groner, B. (1998) Mol Endocrinol 12, 556567

58. Ke, Y., Lesperance, J., Zhang, E. E., Bard-Chapeau, E. A., Oshima, R. G., Muller, W. J., and Feng, G. S. (2006) The Journal of biological chemistry 281, 34374-34380

59. You, M., Flick, L. M., Yu, D., and Feng, G. S. (2001) J Exp Med 193, 101-110

60. Saxton, T. M., and Pawson, T. (1999) Proceedings of the National Academy of Sciences of the United States of America 96, 3790-3795

61. Nguyen, T. V., Ke, Y., Zhang, E. E., and Feng, G. S. (2006) J Immunol 177, 5990-5996

62. Yu, D. H., Qu, C. K., Henegariu, O., Lu, X., and Feng, G. S. (1998) The Journal of biological chemistry 273, 21125-21131

63. Inagaki, K., Noguchi, T., Matozaki, T., Horikawa, T., Fukunaga, K., Tsuda, M., Ichihashi, M., and Kasuga, M. (2000) Oncogene 19, 75-84

64. Manes, S., Mira, E., Gomez-Mouton, C., Zhao, Z. J., Lacalle, R. A., and Martinez, A. C. (1999) Molecular and cellular biology 19, 3125-3135

65. Schoenwaelder, S. M., Petch, L. A., Williamson, D., Shen, R., Feng, G. S., and Burridge, K. (2000) Curr Biol 10, 1523-1526

66. Lacalle, R. A., Mira, E., Gomez-Mouton, C., Jimenez-Baranda, S., Martinez, A. C., and Manes, S. (2002) J Cell Biol 157, 277-289 
67. von Wichert, G., Haimovich, B., Feng, G. S., and Sheetz, M. P. (2003) The EMBO journal 22, 50235035

68. Tartaglia, M., Mehler, E. L., Goldberg, R., Zampino, G., Brunner, H. G., Kremer, H., van der Burgt, I., Crosby, A. H., Ion, A., Jeffery, S., Kalidas, K., Patton, M. A., Kucherlapati, R. S., and Gelb, B. D. (2001) Nature genetics 29, 465-468

69. Roberts, A. E., Araki, T., Swanson, K. D., Montgomery, K. T., Schiripo, T. A., Joshi, V. A., Li, L., Yassin, Y., Tamburino, A. M., Neel, B. G., and Kucherlapati, R. S. (2007) Nature genetics 39, 70-74

70. Tartaglia, M., Martinelli, S., lavarone, I., Cazzaniga, G., Spinelli, M., Giarin, E., Petrangeli, V., Carta, C., Masetti, R., Arico, M., Locatelli, F., Basso, G., Sorcini, M., Pession, A., and Biondi, A. (2005) British journal of haematology 129, 333-339

71. Edouard, T., Montagner, A., Dance, M., Conte, F., Yart, A., Parfait, B., Tauber, M., Salles, J. P., and Raynal, P. (2007) Cellular and molecular life sciences: CMLS 64, 1585-1590

72. Tartaglia, M., Niemeyer, C. M., Fragale, A., Song, X., Buechner, J., Jung, A., Hahlen, K., Hasle, H., Licht, J. D., and Gelb, B. D. (2003) Nature genetics 34, 148-150

73. Niihori, T., Aoki, Y., Ohashi, H., Kurosawa, K., Kondoh, T., Ishikiriyama, S., Kawame, H., Kamasaki, H., Yamanaka, T., Takada, F., Nishio, K., Sakurai, M., Tamai, H., Nagashima, T., Suzuki, Y., Kure, S., Fujii, K., Imaizumi, M., and Matsubara, Y. (2005) Journal of human genetics 50, 192-202

74. Johan, M. F., Bowen, D. T., Frew, M. E., Goodeve, A. C., Wilson, G. A., Peake, I. R., and Reilly, J. T. (2004) British journal of haematology 124, 843-844

75. Cory, S. (1986) Advances in cancer research 47, 189-234

76. Sattler, M., Mohi, M. G., Pride, Y. B., Quinnan, L. R., Malouf, N. A., Podar, K., Gesbert, F., Iwasaki, H., Li, S., Van Etten, R. A., Gu, H., Griffin, J. D., and Neel, B. G. (2002) Cancer cell 1, 479-492

77. Tauchi, T., Feng, G. S., Shen, R., Song, H. Y., Donner, D., Pawson, T., and Broxmeyer, H. E. (1994) The Journal of biological chemistry 269, 15381-15387

78. Mohi, M. G., and Neel, B. G. (2007) Curr Opin Genet Dev 17, 23-30

79. Higashi, H., Tsutsumi, R., Muto, S., Sugiyama, T., Azuma, T., Asaka, M., and Hatakeyama, M. (2002) Science 295, 683-686

80. Tang, C., Luo, D., Yang, H., Wang, Q., Zhang, R., Liu, G., and Zhou, X. (2013) Appl Immunohistochem Mol Morphol

81. Furcht, C. M., Munoz Rojas, A. R., Nihalani, D., and Lazzara, M. J. (2013) Oncogene 32, 2346-2355

82. Jiang, C., Hu, F., Tai, Y., Du, J., Mao, B., Yuan, Z., Wang, Y., and Wei, L. (2012) J Cancer Res Clin Oncol 138, 637-646

83. Bard-Chapeau, E. A., Li, S., Ding, J., Zhang, S. S., Zhu, H. H., Princen, F., Fang, D. D., Han, T., BaillyMaitre, B., Poli, V., Varki, N. M., Wang, H., and Feng, G. S. (2011) Cancer cell 19, 629-639

84. Zhou, X. D., and Agazie, Y. M. (2008) Cell Death Differ 15, 988-996

85. Muenst, S., Obermann, E. C., Gao, F., Oertli, D., Viehl, C. T., Weber, W. P., Fleming, T., Gillanders, W. E., and Soysal, S. D. (2013) Histopathology

86. Dionne, C. A., Jaye, M., and Schlessinger, J. (1991) Ann N Y Acad Sci 638, 161-166

87. Johnson, D. E., and Williams, L. T. (1993) Advances in cancer research 60, 1-41

88. Ullrich, A., Coussens, L., Hayflick, J. S., Dull, T. J., Gray, A., Tam, A. W., Lee, J., Yarden, Y., Libermann, T. A., Schlessinger, J., and et al. (1984) Nature 309, 418-425

89. Garrett, T. P., McKern, N. M., Lou, M., Elleman, T. C., Adams, T. E., Lovrecz, G. O., Kofler, M., Jorissen, R. N., Nice, E. C., Burgess, A. W., and Ward, C. W. (2003) Mol Cell 11, 495-505

90. Ferguson, K. M., Berger, M. B., Mendrola, J. M., Cho, H. S., Leahy, D. J., and Lemmon, M. A. (2003) Mol Cell 11, 507-517

91. Garrett, T. P., McKern, N. M., Lou, M., Elleman, T. C., Adams, T. E., Lovrecz, G. O., Zhu, H. J., Walker, F., Frenkel, M. J., Hoyne, P. A., Jorissen, R. N., Nice, E. C., Burgess, A. W., and Ward, C. W. (2002) Cell 110, 763-773 
92. Ogiso, H., Ishitani, R., Nureki, O., Fukai, S., Yamanaka, M., Kim, J. H., Saito, K., Sakamoto, A., Inoue, M., Shirouzu, M., and Yokoyama, S. (2002) Cell 110, 775-787

93. Badache, A., and Goncalves, A. (2006) J Mammary Gland Biol Neoplasia 11, 13-25

94. Carlsson, J., Nordgren, H., Sjostrom, J., Wester, K., Villman, K., Bengtsson, N. O., Ostenstad, B., Lundqvist, H., and Blomqvist, C. (2004) Br J Cancer 90, 2344-2348

95. Fukushige, S., Matsubara, K., Yoshida, M., Sasaki, M., Suzuki, T., Semba, K., Toyoshima, K., and Yamamoto, T. (1986) Molecular and cellular biology 6, 955-958

96. King, C. R., Kraus, M. H., and Aaronson, S. A. (1985) Science 229, 974-976

97. Pawson, T., and Gish, G. D. (1992) Cell 71, 359-362.

98. Feng, G. S., Shen, R., Heng, H. H., Tsui, L. C., Kazlauskas, A., and Pawson, T. (1994) Oncogene 9, 15451550.

99. Hadari, Y. R., Kouhara, H., Lax, I., and Schlessinger, J. (1998) Molecular and cellular biology 18, 39663973.

100. Lechleider, R. J., Sugimoto, S., Bennett, A. M., Kashishian, A. S., Cooper, J. A., Shoelson, S. E., Walsh, C. T., and Neel, B. G. (1993) The Journal of biological chemistry 268, 21478-21481.

101. Schlessinger, J., Mohammadi, M., Margolis, B., and Ullrich, A. (1992) Cold Spring Harb Symp Quant Biol 57, 67-74

102. Baldys, A., Gooz, M., Morinelli, T. A., Lee, M. H., Raymond, J. R., Jr., Luttrell, L. M., and Raymond, J. R., Sr. (2009) Biochemistry 48, 1462-1473

103. Bollag, G., and McCormick, F. (1991) Nature 351, 576-579

104. Ekman, S., Thuresson, E. R., Heldin, C. H., and Ronnstrand, L. (1999) Oncogene 18, 2481-2488

105. Jiang, X., and Sorkin, A. (2003) Traffic 4, 529-543

106. Pamonsinlapatham, P., Hadj-Slimane, R., Lepelletier, Y., Allain, B., Toccafondi, M., Garbay, C., and Raynaud, F. (2009) Biochimie 91, 320-328

107. Scheffzek, K., Ahmadian, M. R., Kabsch, W., Wiesmuller, L., Lautwein, A., Schmitz, F., and Wittinghofer, A. (1997) Science 277, 333-338

108. Beguinot, L., Lyall, R. M., Willingham, M. C., and Pastan, I. (1984) Proceedings of the National Academy of Sciences of the United States of America 81, 2384-2388

109. Helin, K., and Beguinot, L. (1991) The Journal of biological chemistry 266, 8363-8368

110. Sorkin, A., Di Fiore, P. P., and Carpenter, G. (1993) Oncogene 8, 3021-3028

111. Sorkin, A., and Goh, L. K. (2009) Exp Cell Res 315, 683-696

112. Huang, G., Chantry, A., and Epstein, R. J. (1999) J Cell Biochem 74, 23-30

113. Worthylake, R., Opresko, L. K., and Wiley, H. S. (1999) The Journal of biological chemistry 274, 88658874

114. Debnath, J., Muthuswamy, S. K., and Brugge, J. S. (2003) Methods (San Diego, Calif.) 30, 256-268

115. Agazie, Y. M., Movilla, N., Ischenko, I., and Hayman, M. J. (2003) Oncogene 22, 6909-6918

116. Yuan, Y. L., Zhou, X. H., Song, J., Qiu, X. P., Li, J., and Ye, L. F. (2008) J Laryngol Otol 122, 952-960

117. Duan, L., Miura, Y., Dimri, M., Majumder, B., Dodge, I. L., Reddi, A. L., Ghosh, A., Fernandes, N., Zhou, P., Mullane-Robinson, K., Rao, N., Donoghue, S., Rogers, R. A., Bowtell, D., Naramura, M., Gu, H., Band, V., and Band, H. (2003) The Journal of biological chemistry 278, 28950-28960

118. Longva, K. E., Blystad, F. D., Stang, E., Larsen, A. M., Johannessen, L. E., and Madshus, I. H. (2002) J Cell Biol 156, 843-854

119. Zhou, X., and Agazie, Y. M. (2012) Cell Signal 24, 140-150

120. Hendriks, B. S., Wiley, H. S., and Lauffenburger, D. (2003) Biophys J 85, 2732-2745

121. Muthuswamy, S. K., Gilman, M., and Brugge, J. S. (1999) Molecular and cellular biology 19, 68456857

122. Jiang, X., Huang, F., Marusyk, A., and Sorkin, A. (2003) Mol Biol Cell 14, 858-870

123. Sun, J., Pedersen, M., Bengtsson, S., and Ronnstrand, L. (2007) Exp Cell Res 313, 3935-3942 
124. Aertgeerts, K., Skene, R., Yano, J., Sang, B. C., Zou, H., Snell, G., Jennings, A., Iwamoto, K., Habuka, N., Hirokawa, A., Ishikawa, T., Tanaka, T., Miki, H., Ohta, Y., and Sogabe, S. The Journal of biological chemistry 286, 18756-18765

125. Fan, Y. X., Wong, L., Ding, J., Spiridonov, N. A., Johnson, R. C., and Johnson, G. R. (2008) The Journal of biological chemistry 283, 1588-1596

126. Morandell, S., Stasyk, T., Skvortsov, S., Ascher, S., and Huber, L. A. (2008) Proteomics 8, 4383-4401

127. Ono, M., and Kuwano, M. (2006) Clin Cancer Res 12, 7242-7251

128. Fragale, A., Tartaglia, M., Wu, J., and Gelb, B. D. (2004) Hum Mutat 23, 267-277

129. Meng, S., Chen, Z., Munoz-Antonia, T., and Wu, J. (2005) Biochem J 391, 143-151

130. Ravichandran, K. S., Lorenz, U., Shoelson, S. E., and Burakoff, S. J. (1995) Ann N Y Acad Sci 766, 202203

131. Russo, C., Dolcini, V., Salis, S., Venezia, V., Violani, E., Carlo, P., Zambrano, N., Russo, T., and Schettini, G. (2002) Ann N Y Acad Sci 973, 323-333

132. Schulze, W. X., Deng, L., and Mann, M. (2005) Mol Syst Biol 1, 20050008

133. Monsey, J., Shen, W., Schlesinger, P., and Bose, R. The Journal of biological chemistry 285, 70357044

134. Zhang, X., Gureasko, J., Shen, K., Cole, P. A., and Kuriyan, J. (2006) Cell 125, 1137-1149

135. Yamanashi, Y., Tezuka, T., and Yokoyama, K. J Biochem 151, 353-359

136. Sperka, T., Geissler, K. J., Merkel, U., Scholl, I., Rubio, I., Herrlich, P., and Morrison, H. L. PLoS One 6, e27511

137. Lee-Hoeflich, S. T., Crocker, L., Yao, E., Pham, T., Munroe, X., Hoeflich, K. P., Sliwkowski, M. X., and Stern, H. M. (2008) Cancer Res 68, 5878-5887

138. Rana, P., and Sridhar, S. S. Breast Cancer (Auckl) 6, 67-77

139. Freeman, R. M., Plutzky, J., and Neel, B. G. (1992) Proceedings of the National Academy of Sciences 89, 11239-11239

140. Sugimoto, S., Lechleider, R. J., Shoelson, S. E., Neel, B. G., and Walsh, C. T. (1993) Journal of Biological Chemistry 268, 22771-22771

141. Qu, C. K. (2000) Cell Research 10, 279-288

142. Higashi, H., Nakaya, A., Tsutsumi, R., Yokoyama, K., Fujii, Y., Ishikawa, S., Higuchi, M., Takahashi, A., Kurashima, Y., Teishikata, Y., Tanaka, S., Azuma, T., and Hatakeyama, M. (2004) The Journal of biological chemistry 279, 17205-17216

143. Oh, E. S., Gu, H., Saxton, T. M., Timms, J. F., Hausdorff, S., Frevert, E. U., Kahn, B. B., Pawson, T., Neel, B. G., and Thomas, S. M. (1999) Molecular and cellular biology 19, 3205-3215

144. Qu, C.-K. (2002) Biochimica et biophysica acta 1592, 297-301

145. Tartaglia, M., Kalidas, K., Shaw, A., Song, X., Musat, D. L., van der Burgt, I., Brunner, H. G., Bertola, D. R., Crosby, A., Ion, A., Kucherlapati, R. S., Jeffery, S., Patton, M. A., and Gelb, B. D. (2002) Am J Hum Genet 70, 1555-1563

146. Loh, M. L., Vattikuti, S., Schubbert, S., Reynolds, M. G., Carlson, E., Lieuw, K. H., Cheng, J. W., Lee, C. M., Stokoe, D., Bonifas, J. M., Curtiss, N. P., Gotlib, J., Meshinchi, S., Le Beau, M. M., Emanuel, P. D., and Shannon, K. M. (2004) Blood 103, 2325-2331

147. Grossmann, K. S., Rosario, M., Birchmeier, C., and Birchmeier, W. Advances in Cancer Research 106, 53-89

148. Hanafusa, H., Torii, S., Yasunaga, T., Matsumoto, K., and Nishida, E. (2004) The Journal of biological chemistry 279, 22992-22995

149. Zhang SQ, Y. W., Kontaridis MI, Bivona TG, Wen G, Araki T, Luo J, Thompson JA, Schraven BL, Philips MR, Neel BG. (2004) Mol Cell 13, 15

150. Burks, J., and Agazie, Y. M. (2006) Oncogene 25, 7166-7179

151. Hakak, Y., Hsu, Y. S., and Martin, G. S. (2000) Oncogene 19, 3164-3171 
152. Aceto, N., Sausgruber, N., Brinkhaus, H., Gaidatzis, D., Martiny-Baron, G., Mazzarol, G., Confalonieri, S., Quarto, M., Hu, G., Balwierz, P. J., Pachkov, M., Elledge, S. J., van Nimwegen, E., Stadler, M. B., and Bentires-Alj, M. Nat Med 18, 529-537

153. Yu, D. H., Qu, C. K., Henegariu, O., Lu, X., and Feng, G. S. (1998) The Journal of biological chemistry 273, 21125-21131

154. Qi, J. H., Ito, N., and Claesson-Welsh, L. (1999) The Journal of biological chemistry 274, 14455-14463

155. Schoenwaelder, S. M., Petch, L. A., Williamson, D., Shen, R., Feng, G. S., and Burridge, K. (2000) Curr. Biol. 10, 1523-1526

156. Ivanov, A. V., Peng, H., Yurchenko, V., Yap, K. L., Negorev, D. G., Schultz, D. C., Psulkowski, E., Fredericks, W. J., White, D. E., Maul, G. G., Sadofsky, M. J., Zhou, M.-M., and Rauscher, F. J. (2007) Molecular Cell 28, 823-837

157. Scherr, M., Chaturvedi, A., Battmer, K., Dallmann, I., Schultheis, B., Ganser, A., and Eder, M. (2006) Blood 107, 3279-3287

158. Tao, H., Berno, A. J., Cox, D. R., and Frazer, K. A. (2007) PloS one 2, e697

159. Li, C. C., Kuo, J. C., Waterman, C. M., Kiyama, R., Moss, J., and Vaughan, M. Proc Natl Acad Sci U S A 108, 19228-19233

160. Kelley, L., Hayes, K., Ammer, A., and Martin, K. (2010) PloS one

161. Zhou, X. D., and Agazie, Y. M. (2008) Cell death and differentiation 15, 988-996

162. Price, J. T., Tiganis, T., Agarwal, A., Mechanism, C. d., Djakiew, D., and Thompson, E. W. (1999) Cancer, 5475-5478

163. Ren, Y., Meng, S., Mei, L., Zhao, Z. J., Jove, R., and Wu, J. (2004) The Journal of biological chemistry 279, 8497-8505

164. Zhou, X., and Agazie, Y. M. (2012) Cell Signaling 24, 11

165. Manes, S., Mira, E., Gomez-Mouton, C., Zhao, Z. J., Lacalle, R. A., and Martinez-A, C. (1999) Molecular and cellular biology 19, 3125-3125

166. Matozaki, T., Murata, Y., Saito, Y., Okazawa, H., and Ohnishi, H. (2009) Cancer science 100, 17861793

167. Bryce, N. S., Clark, E. S., Leysath, J. M. L., Currie, J. D., Webb, D. J., and Weaver, A. M. (2005) Current Biology 15, 1276-1285

168. Tsutsumi, R., Takahashi, A., Azuma, T., Higashi, H., and Hatakeyama, M. (2006) Molecular and cellular biology 26, 261-276

169. Slack-Davis, J. K., Martin, K. H., Tilghman, R. W., Iwanicki, M., Ung, E. J., Autry, C., Luzzio, M. J., Cooper, B., Kath, J. C., Roberts, W. G., and Parsons, J. T. (2007) The Journal of biological chemistry 282, 14845-14852

170. Bregeon, J., Loirand, G., Pacaud, P., and Rolli-Derkinderen, M. (2009) American journal of physiology. Cell physiology 297, C1062-1070

171. Lee, H.-H., and Chang, Z.-F. (2008) The Journal of cell biology 181, 999-1012

172. Pintens, S., Neven, P., Drijkoningen, M., Van Belle, V., Moerman, P., Christiaens, M. R., Smeets, A., Wildiers, H., and Vanden Bempt, I. (2009) Journal of clinical pathology 62, 624-628

173. Rakha, E. A., El-Sayed, M. E., Green, A. R., Lee, A. H., Robertson, J. F., and Ellis, I. O. (2007) Cancer $109,25-32$

174. Wells, A. (2006)

175. Martin, V., Botta, F., Zanellato, E., and Molinari, F. Histol Histopathol

176. Lacalle, R. A., Mira, E., Gomez-Mouton, C., Jimenez-Baranda, S., Martinez-A, C., and Manes, S. (2002) The Journal of cell biology 157, 277-289

177. Ren, X. D., Kiosses, W. B., Sieg, D. J., Otey, C. A., Schlaepfer, D. D., and Schwartz, M. A. (2000) J Cell Sci 113 ( Pt 20), 3673-3678

178. Mohi, M. G., and Neel, B. G. (2007) Current opinion in genetics \& development 17, 23-30 
179. Dance, M., Montagner, A., Salles, J.-P., Yart, A., and Raynal, P. (2008) Cellular signalling 20, 453-459

180. Yang, W., Klaman, L. D., Chen, B., Araki, T., Harada, H., Thomas, S. M., George, E. L., and Neel, B. G. (2006) Developmental cell 10, 317-327

181. Yang, Z., Li, Y., and Yin, F. (2008) Exp. Hematol. 36, 1285-1296

182. Chen, L., Pernazza, D., Scott, L. M., Lawrence, H. R., Ren, Y., Luo, Y., Wu, X., Sung, S.-S., Guida, W. C., Sebti, S. M., Lawrence, N. J., and Wu, J. (2010) Biochemical pharmacology, 1-10

183. Chen, L., Sung, S. S., Yip, M. L., Lawrence, H. R., Ren, Y., Guida, W. C., Sebti, S. M., Lawrence, N. J., and $\mathrm{Wu}, \mathrm{J}$. (2006) Molecular pharmacology 70, 562-562

184. Hellmuth, K., Grosskopf, S., Lum, C. T., Wurtele, M., Roder, N., von Kries, J. P., Rosario, M., Rademann, J., and Birchmeier, W. (2008) Proceedings of the National Academy of Sciences of the United States of America 105, 7275-7280

185. Yu, Z.-H., Chen, L., Wu, L., Liu, S., Wang, L., and Zhang, Z.-Y. (2011) Bioorganic \& medicinal chemistry letters 21, 4238-4242

186. Zhang, X., He, Y., Liu, S., Yu, Z., Jiang, Z. X., Yang, Z., Dong, Y., Nabinger, S. C., Wu, L., Gunawan, A. M., and others. (2010) Journal of Medicinal Chemistry 53, 2482-2493

187. Barr, A. J. (2010) Future medicinal chemistry 2, 1563-1576

188. Shultz, L. D., Rajan, V., and Greiner, D. L. (1997) Science 15, 302-307

189. (!!! INVALID CITATION !!!)

190. Ren, L., Chen, X., Luechapanichkul, R., Selner, N. G., Meyer, T. M., Wavreille, A.-S., Chan, R., lorio, C., Zhou, X., Neel, B. G., and Pei, D. (2011) Biochemistry 50, 2339-2356

191. Version, A., Morris, G. M., Goodsell, D. S., Pique, M. E., Lindstrom, W. L., Huey, R., Hart, W. E., Halliday, S., Belew, R., and Olson, A. J. (2010), 1-49

192. Morris, G. M., Goodsell, D. S., Halliday, R. S., Huey, R., Hart, W. E., Belew, R. K., and Olson, A. J. (1998) Journal of Computational Chemistry 19, 1639-1662

193. Ren, L., Chen, X., Luechapanichkul, R., Selner, N. G., Meyer, T. M., Wavreille, A. S., Chan, R., lorio, C., Zhou, X., Neel, B. G., and Pei, D. (2011) Biochemistry 50, 2339-2356

194. O'Reilly, A. M., and Neel, B. G. (1998) Molecular and cellular biology 18, 161-177

195. Tenev, T., Keilhack, H., Tomic, S., Stoyanov, B., Stein-Gerlach, M., Lammers, R., Krivtsov, A. V., Ullrich, A., and Bohmer, F. D. (1997) The Journal of biological chemistry 272, 5966-5973

196. Montalibet, J., Skorey, K. I., and Kennedy, B. P. (2005) Methods (San Diego, Calif.) 35, 2-8

197. Hartman, Z. R., Schaller, M. D., and Agazie, Y. M. (2013) Molecular cancer research : MCR 11, 651664

198. Barr, A. J., Ugochukwu, E., Lee, W. H., King, O. N., Filippakopoulos, P., Alfano, I., Savitsky, P., BurgessBrown, N. A., Muller, S., and Knapp, S. (2009) Cell 136, 352-363

199. Hellmuth, K., Grosskopf, S., Lum, C. T., Wurtele, M., Roder, N., von Kries, J. P., Rosario, M., Rademann, J., and Birchmeier, W. (2008) Proceedings of the National Academy of Sciences of the United States of America 105, 7275-7280

200. Sweeney, M. C., Wavreille, A.-S., Park, J., Butchar, J. P., Tridandapani, S., and Pei, D. (2005) Biochemistry 44, 14932-14947

201. Slamon, D. J., Leyland-Jones, B., Shak, S., Fuchs, H., Paton, V., Bajamonde, A., Fleming, T., Eiermann, W., Wolter, J., Pegram, M., Baselga, J., and Norton, L. (2001) N Engl J Med 344, 783-792

202. Baselga, J., Bradbury, I., Eidtmann, H., Di Cosimo, S., de Azambuja, E., Aura, C., Gomez, H., Dinh, P., Fauria, K., Van Dooren, V., Aktan, G., Goldhirsch, A., Chang, T. W., Horvath, Z., Coccia-Portugal, M., Domont, J., Tseng, L. M., Kunz, G., Sohn, J. H., Semiglazov, V., Lerzo, G., Palacova, M., Probachai, V., Pusztai, L., Untch, M., Gelber, R. D., and Piccart-Gebhart, M. (2012) Lancet 379, 633-640

203. Jenab-Wolcott, J., and Giantonio, B. J. (2009) Expert opinion on biological therapy 9, 507-517

204. Chan, G., Kalaitzidis, D., and Neel, B. G. (2008) Cancer metastasis reviews 27, 179-192

205. Wolf, K., and Friedl, P. (2011) Trends in cell biology 21, 736-744 
206. Lauffenburger, D. A., and Horwitz, A. F. (1996) Cell 84, 359-369

207. Shan, Y., Yu, L., Li, Y., Pan, Y., Zhang, Q., Wang, F., Chen, J., and Zhu, X. (2009) PLoS biology 7, e1000116

208. Barr, A. J. (2011) Future medicinal chemistry 2, 1563-1576

209. Chen, L., Sung, S. S., Yip, M. L., Lawrence, H. R., Ren, Y., Guida, W. C., Sebti, S. M., Lawrence, N. J., and Wu, J. (2006) Molecular pharmacology 70, 562-570

210. Hegazy, S. A., Wang, P., Anand, M., Ingham, R. J., Gelebart, P., and Lai, R. (2010) The Journal of biological chemistry 285, 19813-19820

211. Zhang, X., He, Y., Liu, S., Yu, Z., Jiang, Z. X., Yang, Z., Dong, Y., Nabinger, S. C., Wu, L., Gunawan, A. M., Wang, L., Chan, R. J., and Zhang, Z. Y. (2010) Journal of medicinal chemistry 53, 2482-2493

212. Mali, R. S., Ma, P., Zeng, L. F., Martin, H., Ramdas, B., He, Y., Sims, E., Nabinger, S., Ghosh, J., Sharma, N., Munugalavadla, V., Chatterjee, A., Li, S., Sandusky, G., Craig, A. W., Bunting, K. D., Feng, G. S., Chan, R. J., Zhang, Z. Y., and Kapur, R. (2012) Blood 120, 2669-2678

213. Xu, J., Zeng, L. F., Shen, W., Turchi, J. J., and Zhang, Z. Y. (2013) Biochemical and biophysical research communications

214. Dechert, U., Adam, M., Harder, K. W., Clark-Lewis, I., and Jirik, F. (1994) The Journal of biological chemistry 269, 5602-5611

215. Amin, H. M., Hoshino, K., Yang, H., Lin, Q., Lai, R., and Garcia-Manero, G. (2007) The Journal of pathology 212, 402-410

216. Bittorf, T., Seiler, J., Zhang, Z., Jaster, R., and Brock, J. (1999) Biological chemistry 380, 1201-1209

217. Mena-Duran, A. V., Togo, S. H., Bazhenova, L., Cervera, J., Bethel, K., Senent, M. L., Nieva, J., Sanz, G. F., Sanz, M. A., Saven, A., and Mustelin, T. (2005) British journal of haematology 129, 791-794

218. Oka, T., Ouchida, M., Koyama, M., Ogama, Y., Takada, S., Nakatani, Y., Tanaka, T., Yoshino, T., Hayashi, K., Ohara, N., Kondo, E., Takahashi, K., Tsuchiyama, J., Tanimoto, M., Shimizu, K., and Akagi, T. (2002) Cancer Res 62, 6390-6394 


\section{8) Curriculum Vitae}

\section{ZACH HARTMAN}

Phone: (304) 904-9104

zhartma1@mix.wvu.edu

\section{Education}

12 Northwest Ct.

Morgantown, WV 26505

Ph.D West Virginia University (currently pursuing)

August 2008 to 2013

Major: Biochemistry and Molecular Biology

B.Sc. West Liberty State College, Chemistry

August 2004 to May 2008

Major: Chemistry

Graduated Summa Cum Laude

\section{$\underline{\text { Research Experience }}$}

West Virginia University School of Medicine

2008 to Present

Research Fellow, Yehenew Agazie, Ph.D.

- Characterize the tyrosine phosphatase Shp2 as a therapeutic target in breast cancer

- Use computational and biochemical techniques to elucidate Shp2 substrate selectivity

West Virginia University School of Pharmacy

Research Assistant, Peter Gannett, Ph.D.

- Energy calculations of substrate-substrate interactions within the active site of Cytochrome P450 2C9 using molecular modeling techniques

West Liberty State College

2005-2008

Research Assistant, Jarrett Aguilar, Ph.D. 
- Molecular modeling of dynamic enzyme-substrate interactions of Cytochrome P450 2C9 using flurbiprofen and various dapsone analogs to explain molecular interactions contributing to atypical kinetics

\section{$\underline{\text { Publications }}$}

"The tyrosine phosphatase SHP2 regulates focal adhesion kinase to promote EGF-induced lamellipodia persistence and cell migration" Molecular Cancer Research, 2013

"HER2 Stabilizes EGFR and Itself by Altering Autophosphorylation Patterns in a Manner That Overcomes Regulatory Mechanisms and Promotes Proliferative and Transformation Signaling" Oncogene, 2012

\section{Fellowships, awards, and other honors}

Department of Defense Congressionally Directed Medical Research Programs Breast Cancer Research Program Predoctoral Traineeship Award 2011 to Present

Title: "Characterizing SHP2 as a novel therapeutic target in breast cancer"

\section{$\underline{\text { Poster Presentations }}$}


Title: "Exploring a mechanism for Shp2 control of cell motility"

American Association for Cancer Research Breast Cancer Symposium

December 2011

Title: "Molecular mechanism for Src homology phosphotyrosyl phosphatase 2 regulation of cell motility and migration"

American Association for Cancer Research Breast Cancer Symposium

December 2011

Title: "The role of Src homology phosphotyrosyl phosphatase-2 in basal-type/triple-negative breast cancer implications for targeted therapy"

American Association for Cancer Research 102nd Annual Meeting

April 2011

Title: "Inhibition of SHP2 abolishes mammary tumorigenesis in mice"

Van Liere Research Day Poster Competition

March 2011

Title: "Exploring the molecular mechanism of SHP2 regulation of cell motility and invasion"

Van Liere Research Day Poster Competition

April 2010

Title: "Development of a model for SHP-2 specificity and substrate prediction"

American Chemistry Society National Meeting

May 2006

Title: "Molecular dynamics study of hydrogen bonding dependence of flurbiprofen and dapsone with the Cytochrome P450 2C9"

\section{Speaking Engagements}

Van Liere Research Day Oral Presentation Competition

March 2012 
Title: "Exploring a mechanism for Shp2 control of cell motility"

Dissertation Proposal Defense

March 2011

Title: "Characterizing SHP2 as a therapeutic target in breast cancer"

Van Liere Research Day Oral Presentation Competition

March 2011

Title: "Exploring the molecular mechanism of SHP2 regulation of cell motility and invasion"

Cytoskeleton/Migration/Signaling Group Meeting Invited Talk

October 2010

Title: "Exploring the substrate specificity of Shp2"

Cancer Cell Biology Student Meeting Invited Talk

August 2010

Title: "Shp2: role and targeting in breast cancer"

Meeting of the Behavioral and Biomedical Sciences Invited Talk

July 2010

Title: "Happyhour, a Ste20 family kinase, implicates EGFR signaling in ethanol-induced behaviors"

\section{Teaching Experience}

Tutor.com Math and Chemistry Tutor

2008 to Present

Responsibilities: Tutor students online in both high school and college-level mathematics, chemistry, biochemistry, and biology

Cell Biology Methods Course Coordinator and Instructor

Fall 2011, Fall 2012 
Responsibilities: Design and organize lectures in conjunction with other students to facilitate a course to introduce first-year graduate students to methodologies in cell biology

Lectures given: Protein Purification, Cell Culture, Structure Determination, and Electrophoresis

West Virginia University Tutor

$2009-2010$

Responsibilities: Assist first-year graduate students struggling in the topics of biochemistry and cell biology 